\title{
Revised American Thyroid Association Guidelines for the Management of Medullary Thyroid Carcinoma
}

\author{
The American Thyroid Association Guidelines Task Force \\ on Medullary Thyroid Carcinoma
}

\begin{abstract}
Samuel A. Wells, Jr., ${ }^{1, *}$ Sylvia L. Asa,${ }^{2}$ Henning Dralle, ${ }^{3}$ Rossella Elisei, ${ }^{4}$ Douglas B. Evans, ${ }^{5}$ Robert F. Gagel, ${ }^{6}$ Nancy Lee, ${ }^{7}$ Andreas Machens, ${ }^{3}$ Jeffrey F. Moley, ${ }^{8}$ Furio Pacini, ${ }^{9}$ Friedhelm Raue, ${ }^{10}$ Karin Frank-Raue, ${ }^{10}$ Bruce Robinson,, ${ }^{11}$. Sara Rosenthal, ${ }^{12}$ Massimo Santoro, ${ }^{13}$ Martin Schlumberger, ${ }^{14}$ Manisha Shah, ${ }^{15}$ and Steven G. Waguespack ${ }^{6}$
\end{abstract}

Introduction: The American Thyroid Association appointed a Task Force of experts to revise the original Medullary Thyroid Carcinoma: Management Guidelines of the American Thyroid Association.

Methods: The Task Force identified relevant articles using a systematic PubMed search, supplemented with additional published materials, and then created evidence-based recommendations, which were set in categories using criteria adapted from the United States Preventive Services Task Force Agency for Healthcare Research and Quality. The original guidelines provided abundant source material and an excellent organizational structure that served as the basis for the current revised document.

Results: The revised guidelines are focused primarily on the diagnosis and treatment of patients with sporadic medullary thyroid carcinoma (MTC) and hereditary MTC.

Conclusions: The Task Force developed 67 evidence-based recommendations to assist clinicians in the care of patients with MTC. The Task Force considers the recommendations to represent current, rational, and optimal medical practice.

\section{OVERVIEW}

A CCORDING TO CURRENT Surveillance, Epidemiology, and End Results (SEER) data, medullary thyroid carcinoma (MTC) accounts for $1 \%-2 \%$ of thyroid cancers in the United States, a much lower range than frequently cited (3\%-5\%), primarily due to the marked increase in the relative incidence of papillary thyroid carcinoma (PTC) over the last three de- cades (1). Advances in the basic research and clinical investigation of MTC reported in specialty publications of endocrinology, genetics, nuclear medicine, oncology, pathology, pediatrics, radiology, and surgery make it challenging for clinicians to remain current on new developments. Several academic organizations have published guidelines for the management of patients with MTC (2-4). In 2007 the American Thyroid Association (ATA) assembled a group of

\footnotetext{
${ }^{1}$ Genetics Branch, National Cancer Institute, National Institutes of Health, Bethesda, Maryland.

${ }^{2}$ Department of Pathology, University Health Network, and Department of Laboratory Medicine and Pathobiology, University of Toronto, Toronto, Ontario, Canada.

${ }^{3}$ Department of General, Visceral, and Vascular Surgery, University Hospital, University of Halle-Wittenberg, Halle/Saale, Germany.

${ }^{4}$ Department of Endocrinology, University of Pisa, Pisa, Italy.

${ }^{5}$ Department of Surgery, Medical College of Wisconsin, Milwaukee, Wisconsin.

${ }^{6}$ Department of Endocrine Neoplasia and Hormonal Disorders, Division of Internal Medicine, The University of Texas MD Anderson Cancer Center, Houston, Texas.

${ }^{7}$ Department of Radiation Oncology, Memorial Sloan-Kettering Cancer Center, New York, New York.

${ }^{8}$ Department of Surgery, Washington University School of Medicine, St. Louis, Missouri.

${ }^{9}$ Section of Endocrinology and Metabolism, Department of Internal Medicine, Endocrinology and Metabolism and Biochemistry, University of Siena, Policlinico Santa Maria alle Scotte, Siena, Italy.

${ }^{10}$ Endocrine Practice, Moleculargenetic Laboratory, Medical Faculty, University of Heidelberg, Heidelberg, Germany.

${ }^{11}$ University of Sydney School of Medicine, Sydney, New South Wales, Australia.

${ }^{12}$ Departments of Internal Medicine, Pediatrics and Behavioral Science, University of Kentucky, Lexington, Kentucky.

${ }^{13}$ Dipartimento di Medicina Molecolare e Biotecnologie Mediche, Universita' di Napoli “Federico II," Napoli, Italy.

${ }^{14}$ Institut Gustave Roussy, Service de Medecine Nucleaire, Université of Paris-Sud, Villejuif, France.

${ }^{15}$ Division of Medical Oncology, Department of Internal Medicine, The Ohio State University, Columbus, Ohio.

*Task Force Chairperson; Authorship listed in alphabetical order following Chair.
} 
expert clinicians and basic scientists to evaluate published papers and to recommend evidence-based guidelines for the diagnosis and management of patients with MTC. The guidelines were published in 2009 (5). The current document is the first revision of the original guidelines, and it is hoped that it will assist clinicians of all specialties in the management of patients with MTC. It is not the intent of the guidelines to replace the individual physician's decision-making or the wishes of the patient or the patient's family.

\section{Methods}

\section{Presentation of results and recommendations}

Table 1 lists the topics addressed by the Guidelines Task Force (Task Force). Specific recommendations (R) regarding patient management are numbered in the body of the guidelines. The location key can be used if viewing the guidelines in a file or web page. Each location key is unique and can be copied into the Find or Search function to navigate to the section of interest. Table 2 lists the abbreviations used throughout the document.

\section{Administration}

The ATA Board of Directors selected a Task Force Chairman based on clinical experience with MTC and the absence of dogmatically held views in areas of recognized controversy. Task Force members were selected based on clinical and research expertise and included international scientists from the fields of endocrinology, ethics, genetics, molecular biology, medical oncology, pathology, pediatrics, nuclear medicine, radiation oncology, and surgery. All Task Force members disclosed potential conflicts of interest.

Teams of Task Force members reviewed and revised specific sections of the original document. The teams' rec- ommendations were the basis for a preliminary draft of the revised guidelines. After subsequent revisions and critical reviews of a series of drafts the Task Force developed a final document. The consensus was most often unanimous; however, on some issues there were disparate views among Task Force members, which are noted in the document. The ATA Board of Directors approved the final document, and it was subsequently endorsed by the American Academy of Pediatrics; American Association of Clinical Endocrinologists; American Association of Endocrine Surgeons; American Head \& Neck Society; American Society of Pediatric Hematology/Oncology; Australian and New Zealand Endocrine Surgeons; British Association of Head and Neck Oncologists; British Nuclear Medicine Society; European Thyroid Association; International Association of Endocrine Surgeons; International Federation of Head and Neck Oncologic Societies; Italian Endocrine Society; Korean Society of ThyroidHead and Neck Surgery; Latin American Thyroid Society; Pediatric Endocrine Society; and The Endocrine Society.

\section{Literature review and evidence-based medicine}

The Task Force identified relevant articles by searching MEDLINE/PubMed from January 1980 to April 2014 using the following search terms: calcitonin, medullary carcinoma, medullary thyroid cancer, multiple endocrine neoplasia, multiple endocrine neoplasia type $2 \mathrm{~A}$, multiple endocrine neoplasia type $2 \mathrm{~B}, R E T$, and thyroid cancer. Task Force members also provided additional relevant articles, book chapters, and other materials. The Task Force members graded recommendations using criteria adapted from the United States Preventive Services Task Force, Agency for Healthcare Research and Quality (Table 3) as were used in the previous MTC guidelines (5).

Table 1. Organization of Revised American Thyroid Association Guidelines for the Management of Medullary Thyroid Carcinoma

[C] Clinical characteristics and relationship between genotype and phenotype in patients with sporadic MTC and patients with hereditary MTC sporadic MTC

[E] Ethical considerations for genetic screening

[E-1] Adults

[E-2] The pediatric population

$12 \quad 576$


TABle 1. (CONTINUED)

\section{Section/subsection}

Location

[F] Secretory products of MTC

[F-1] Calcitonin

[F-2] Carcinoembryonic antigen

[G] Morphological examination of an excisional biopsy or the thyroidectomy specimen

$[\mathrm{H}]$ The diagnosis of MTC in patients presenting with a thyroid nodule

[H-1] Fine-needle aspiration biopsy

[H-2] Measurement of the serum Ctn level in patients with nodular thyroid disease

[I] Management of patients with a thyroid nodule and histological documentation of MTC

[I-1] Preoperative imaging studies

[I-2] The initial surgical treatment of patients with MTC

[J] Results of thyroidectomy in patients with MTC

[K] Management of patients with locally advanced or metastatic MTC

[L] Management of patients following an incomplete thyroidectomy and lymph node dissection

[M] Management of normal parathyroid glands resected or devascularized during surgery

[N] Hormone replacement following thyroidectomy

[O] Prophylactic thyroidectomy in children with hereditary MTC

[O-1] Prophylactic thyroidectomy for children with MEN2A

[O-2] Prophylactic thyroidectomy for children with MEN2B

[P] Management of PHEO in patients with MEN2A and MEN2B

[Q] Management of HPTH in patients with MEN2A

[R] Evaluation of patients following thyroidectomy

[R-1] Measurement of doubling times of serum Ctn and CEA to determine rate of progression of MTC

[S] Treatment of patients with regional metastatic MTC

[S-1] Neck exploration

[S-2] Role of postoperative radioiodine ablation

[S-3] Adjunctive EBRT to the neck

[T] Evaluation of patients with distant metastases

[T-1] Role of open or laparoscopic evaluation of the liver and selective venous catheterization with measurement of hepatic and peripheral vein stimulated Ctn levels

[U] Diagnosis and treatment of patients with clinically evident metastatic disease

[U-1] Brain metastases

[U-2] Bone metastases

[U-3] Lung and mediastinal metastases

[U-4] Hepatic metastases

[U-5] Cutaneous metastases

[U-6] Palliation of patients with advanced MTC

[V] Systemic therapy

[V-1] The basis for targeted therapy with TKIs

[V-1-1] Clinical trials of vandetanib in patients with advanced MTC

[V-1-2] Clinical trials of cabozantinib in patients with advanced MTC

[W] Treatment of patients with hormonally active metastases

[W-1] Diarrhea

[W-2] Ectopic Cushing's syndrome

$R$ number (page)

$\begin{array}{cc} & 576 \\ 13,14 & 576-577 \\ 15 & 577 \\ 16-18 & 578 \\ & 578 \\ 19 & 578-580 \\ 20 & 580 \\ & 580 \\ 21-23 & 580-581 \\ 24-26 & 581-582 \\ & 582-583 \\ 27 & 583 \\ 28,29 & 583 \\ 30 & 583-584 \\ 31,32 & 584 \\ & 584-585 \\ & 585-586 \\ 33-36 & 586-587 \\ 37-41 & 587-588 \\ 42-44 & 588 \\ 45-48 & 588-590 \\ 49 & 590-591\end{array}$

$\begin{array}{cc} & 591 \\ 50 & 591 \\ 51 & 591 \\ 52 & 591-592\end{array}$

592

$53,54 \quad 592$

$\begin{array}{cc}55 & 592-593 \\ 56-58 & 593 \\ 59 & 593 \\ 60 & 593 \\ 61 & 593-594 \\ 62 & 594 \\ 63,64 & 594 \\ & 594 \\ & 594-595 \\ 65 & 595 \\ & 595 \\ 66 & 595-596 \\ 67 & 596\end{array}$

\section{[A] BACKGROUND}

Over 100 years ago Jacquet described a thyroid tumor with amyloid; however, it was not until 1959 that Hazard and associates provided a definitive histological description of medullary thyroid carcinoma (MTC) and so named it $(6,7)$. Williams discovered that MTC originated from the neural crest derived parafollicular C-cells of the thyroid gland (8). Tashjian and colleagues discovered that the C-cells secrete the polypeptide calcitonin ( $\mathrm{Ctn}$ ), and they and subsequently others showed that intravenously administered calcium, pentagastrin, or both together, are potent Ctn secretagogues
$(9,10)$. Shortly after the discovery that MTC represents a unique thyroid cancer, it was recognized that the tumor occurred either sporadically or in a hereditary form as a component of the type 2 multiple endocrine neoplasia (MEN) syndromes, MEN2A and MEN2B, and the related syndrome, familial MTC (FMTC).

\section{[B] ETIOLOGY OF SPORADIC AND HEREDITARY MTC}

The RET protooncogene, located on chromosome 10q11.2, encodes a single-pass transmembrane receptor of the tyrosine kinase family. RET is expressed in cells derived from the 
Table 2. Abbreviations Used for Medullary Thyroid Cancer Management Guidelines

\begin{tabular}{|c|c|}
\hline ACTH & Adrenocorticotropic hormone \\
\hline AJCC & American Joint Committee on Cancer \\
\hline ATA & American Thyroid Association \\
\hline $\mathrm{CCH}$ & C-cell hyperplasia \\
\hline CEA & Carcinoembryonic antigen \\
\hline CLA & Cutaneous lichen amyloidosis \\
\hline $\mathrm{CRH}$ & Corticotropin-releasing hormone \\
\hline $\mathrm{CT}$ & Computed tomography (tomographic) \\
\hline Ctn & Calcitonin \\
\hline EBRT $^{\mathrm{a}}$ & External beam radiation therapy \\
\hline EMA & European Medicines Agency \\
\hline FDA & U.S. Food and Drug Administration \\
\hline FDG-PET & $\begin{array}{l}\text { 2-[Fluorine-18]fluoro-2-deoxy-D-glucose- } \\
\text { positron emission tomography }\end{array}$ \\
\hline F-DOPA & ${ }^{18} \mathrm{~F}$-dihydroxyphenylalanine \\
\hline FMTC & Familial medullary thyroid cancer \\
\hline FNA & Fine-needle aspiration \\
\hline FTC & Follicular thyroid carcinoma \\
\hline HD & Hirschsprung's disease \\
\hline HIPAA & $\begin{array}{l}\text { Health Insurance Portability and } \\
\text { Accountability Act }\end{array}$ \\
\hline HPTH & Hyperparathyroidism \\
\hline HR & Hazard ratio \\
\hline ICMA & Immunochemiluminometric assay \\
\hline IHC & Immunohistochemical \\
\hline IMRT & Intensity-modulated radiation therapy \\
\hline MEN & Multiple endocrine neoplasia \\
\hline MIBG & Metaiodobenzylguanidine \\
\hline MRI & Magnetic resonance imaging \\
\hline MTC & Medullary thyroid carcinoma \\
\hline NCT & National Clinical Trial \\
\hline OMIM & Online Mendelian Inheritance in Man \\
\hline PEG & Percutaneous gastrostomy \\
\hline PFS & Progression-free survival \\
\hline PGD & Preimplantation genetic diagnosis \\
\hline PHEO & Pheochromocytoma \\
\hline PTC & Papillary thyroid carcinoma \\
\hline PTH & Parathyroid hormone \\
\hline RAI & Radioactive iodine \\
\hline RECIST & Response evaluation criteria in solid tumors \\
\hline REMS & Risk evaluation and mitigation strategies \\
\hline$R E T$ & $\begin{array}{l}\text { REarranged during Transfection } \\
\text { protooncogene }\end{array}$ \\
\hline SEER & Surveillance, Epidemiology, and End Results \\
\hline TKI & Tyrosine kinase inhibitor \\
\hline TNM & Tumor, node, metastases \\
\hline TSH & Thyrotropin \\
\hline US & Ultrasound \\
\hline VEGF & Vascular endothelial growth factor \\
\hline
\end{tabular}

${ }^{\mathrm{a}}$ May include intensity-modulated radiation therapy.

neural crest, the branchial arches, and the urogenital system $(11,12)$. Takahashi and associates discovered the RET (REarranged during Transfection) oncogene in 1985 (13). Within less than a decade following this observation it was found that virtually all patients with MEN2A, MEN2B, and FMTC have RET germline mutations and approximately $50 \%$ of sporadic MTCs have somatic RET mutations (14-19). Investigators recently discovered that $18 \%-80 \%$ of sporadic MTCs lacking somatic RET mutations have somatic mutations of HRAS, KRAS, or rarely NRAS (20-22). Subsequent exomic sequencing studies of MTCs detected no additional common genetic mutations (23).
The somatic RET codon M918T mutation in sporadic MTC appears to portend an aggressive clinical course and a poor prognosis $(24,25)$. In a recent study of 160 patients with sporadic MTC the prevalence of somatic RET codon M918T mutations varied depending on tumor size: $<1 \mathrm{~cm}, 6(11.3 \%)$ of 53 patients; $1-2 \mathrm{~cm}, 8(11.8 \%)$ of 68 patients; $2-3 \mathrm{~cm}, 7$ (31.8\%) of 22 patients; and $>3 \mathrm{~cm}, 10(58.8 \%)$ of 17 patients (26). These data raise the question of whether RET acts alone as the initiator of oncogenesis in sporadic MTC or is activated later as a driver of tumor growth, with other genes playing a significant role in MTC onset. An alternate explanation for these findings is that M918T mutated tumors have a high growth rate and are more likely to be diagnosed when they are larger. Also, an important technical aspect of the study was that the sensitivity of the mutation calling was only $30 \%$. Furthermore, the low prevalence of the M918T mutation in microcarcinomas may represent a different entity such as carcinoma in situ; precisely because it is not driven by RET.

$R E T$ is a remarkable oncogene that is not only central to the development of sporadic and hereditary MTC but to other malignant and nonmalignant diseases as well. Chromosomal translocations activating RET occur in $20 \%-30 \%$ of patients with PTC (27). Activating RET translocations also occur, but much less frequently, in patients with lung adenocarcinoma and chronic myelomonocytic leukemia $(28,29)$. Furthermore, inactivating mutations occur throughout the RET oncogene in patients with hereditary and sporadic Hirschsprung's disease (HD) $(30,31)$.

At the Seventh International Workshop on MEN a group of experienced clinicians and basic scientists developed the first guidelines for managing patients with hereditary MTC (2). Subsequently, with the discovery of additional oncogenic RET mutations and their associated phenotypes, it became clear that the original guidelines needed modification. Recently, the North American Neuroendocrine Tumor Society, the National Comprehensive Cancer Network, and the American Thyroid Association (ATA) published guidelines for the management of patients with sporadic MTC and hereditary MTC (3-5). Each of the four guidelines described the disease phenotypes associated with specific RET mutations in hereditary MTC and recommended timing of early thyroidectomy based on the specific RET mutation. Three of the groups used either the TNM designation of the American Joint Committee on Cancer (AJCC), or terms such as Level I, II, or III, or "high," "higher," or "highest," to designate progressive increases in aggressiveness of the MTC (2-4). The aggressiveness was based on the development of MTC at an early age, frequently in association with metastatic disease. The original ATA Guidelines used A, B, C, and D designations to define categories of RET mutations associated with increasing aggressiveness (from A to D) of the MTC (5).

There has been confusion regarding the different ATA risk categories. Therefore, the Task Force recommends that category D be changed to a new category, "highest risk" (HST); category $\mathrm{C}$ be changed to a new category, "high risk" $(\mathrm{H})$; and the A and B categories be combined into a new category, "moderate risk" (MOD). The ATA-HST category includes patients with MEN2B and the RET codon M918T mutation, the ATA-H category includes patients with RET codon C634 mutations and the RET codon A883F mutation, and the ATAMOD category includes patients with RET codon mutations other than M918T, C634, and A883F. 
Table 3. Strength of Recommendations Based on Available Evidence

\begin{tabular}{l} 
Rating Definition \\
\hline A
\end{tabular} $\begin{aligned} & \text { Strongly recommends. The recommendation is based on good evidence that the service or intervention can } \\
& \text { improve important health outcomes. Evidence includes consistent results from well-designed, } \\
& \text { well-conducted studies in representative populations that directly assess effects on health outcomes. } \\
& \text { Recommends. The recommendation is based on fair evidence that the service or intervention can improve } \\
& \text { important health outcomes. The evidence is sufficient to determine effects on health outcomes, but the } \\
& \text { strength of the evidence is limited by the number, quality, or consistency of the individual studies; } \\
& \text { generalizability to routine practice; or indirect nature of the evidence on health outcomes. } \\
& \text { Recommends. The recommendation is based on expert opinion. } \\
& \text { C } \\
& \text { Recommends against. The recommendation is based on expert opinion. } \\
& \text { E } \\
& \text { Recommends against. The recommendation is based on fair evidence that the service or intervention does } \\
& \text { not improve important health outcomes or that harms outweigh benefits. } \\
& \text { F } \\
& \text { Strongly recommends against. The recommendation is based on good evidence that the service or } \\
& \text { intervention does not improve important health outcomes or that harms outweigh benefits. } \\
& \text { I } \\
& \text { Recommends neither for nor against. The panel concludes that the evidence is insufficient to recommend } \\
& \text { for or against providing the service or intervention because evidence is lacking that the service or } \\
& \text { intervention improves important health outcomes, the evidence is of poor quality, or the evidence is } \\
& \text { conflicting. As a result, the balance of benefits and harms cannot be determined. }\end{aligned}$

Adapted from the U.S. Preventive Services Task Force, Agency for Healthcare Research and Quality.

Since the discovery of the RET oncogene, over 100 mutations, duplications, insertions, or deletions involving $R E T$ have been identified in patients with hereditary MTC. The most common RET germline mutations causing MEN2A and MEN2B and the clinical aggressiveness of the MTC associated with the mutations are shown in Table 4 . A complete tabulation of RET germline mutations reported to date, including single or multiple mutations, duplications, insertions or deletions, and chromosomal rearrangements involving $R E T$, can be found in the Supplementary Information (Supplementary Data are available online at www.liebertpub.com/ thy) and at the continually updated ARUP database website: www.arup.utah.edu/database/MEN2/MEN2_welcome.php (32).
The risk designation for sporadic MTC is based on the AJCC tumor $(\mathrm{T})$, node $(\mathrm{N})$, and metastases $(\mathrm{M})$ categories.

\section{- RECOMMENDATION 1}

The current ATA risk categories for hereditary MTC should be changed. The current level D category should be changed to a new category, "highest risk" (HST) that includes patients with MEN2B and the RET codon M918T mutation. The current level C category should be changed to a new category, "high risk" (H) that includes patients with the RET codon C634 mutations and the RET codon A883F mutation. The current level A and B categories should be combined

Table 4. Relationship of Common RET Mutations to Risk of Aggressive MTC in MEN2A AND MEN2B, AND TO THE INCIDENCE OF PHEO, HPTH, CLA, AND HD IN MEN2A

\begin{tabular}{|c|c|c|c|c|c|c|}
\hline RET mutation ${ }^{\mathrm{a}}$ & Exon & MTC risk level ${ }^{\mathrm{b}}$ & Incidence of $P H E O^{\mathrm{c}}$ & Incidence of $H P T H^{\mathrm{c}}$ & $C L A^{\mathrm{d}}$ & $H D^{\mathrm{d}}$ \\
\hline G533C & 8 & MOD & + & - & $\mathrm{N}$ & $\mathrm{N}$ \\
\hline C609F/G/R/S/Y & 10 & MOD & $+/++$ & + & $\mathrm{N}$ & Y \\
\hline C611F/G/S/Y/W & 10 & MOD & $+/++$ & + & $\mathrm{N}$ & Y \\
\hline C618F/R/S & 10 & MOD & $+/++$ & + & $\mathrm{N}$ & Y \\
\hline C620F/R/S & 10 & MOD & $+/++$ & + & $\mathrm{N}$ & Y \\
\hline C630R/Y & 11 & MOD & $+/++$ & + & $\mathrm{N}$ & $\mathrm{N}$ \\
\hline D631Y & 11 & MOD & +++ & - & $\mathrm{N}$ & $\mathrm{N}$ \\
\hline C634F/G/R/S/W/Y & 11 & $\mathrm{H}$ & +++ & ++ & Y & $\mathrm{N}$ \\
\hline K666E & 11 & MOD & + & - & $\mathrm{N}$ & $\mathrm{N}$ \\
\hline E768D & 13 & MOD & - & - & $\mathrm{N}$ & $\mathrm{N}$ \\
\hline L790F & 13 & MOD & + & - & $\mathrm{N}$ & $\mathrm{N}$ \\
\hline V804L & 14 & MOD & + & + & $\mathrm{N}$ & $\mathrm{N}$ \\
\hline V804M & 14 & MOD & + & + & $\mathrm{Y}$ & $\mathrm{N}$ \\
\hline A883F & 15 & $\mathrm{H}$ & +++ & - & $\mathrm{N}$ & $\mathrm{N}$ \\
\hline S891A & 15 & MOD & + & + & $\mathrm{N}$ & $\mathrm{N}$ \\
\hline R912P & 16 & MOD & - & - & $\mathrm{N}$ & $\mathrm{N}$ \\
\hline M918T & 16 & HST & +++ & - & $\mathrm{N}$ & $\mathrm{N}$ \\
\hline
\end{tabular}

${ }^{a}$ The references for each of the RET mutations can be found in the Supplementary Information, where all reported RET mutations in MTC are listed.

${ }^{\mathrm{b}}$ Risk of aggressive MTC: MOD, moderate; H, high; HST, highest.

${ }^{\mathrm{c}}$ Incidence of PHEO and HPTH: $+=\sim 10 \% ;++=\sim 20 \%-30 \% ;+++=\sim 50 \%$.

${ }^{\mathrm{d}} \mathrm{Y}$, positive occurrence; $\mathrm{N}$, negative occurrence. 
into a new category "moderate risk" (MOD) that include patients with hereditary MTC and RET codon mutations other than M918T, C634, and A883F. Grade C Recommendation

\section{[C] CLINICAL CHARACTERISTICS AND RELATIONSHIP BETWEEN GENOTYPE AND PHENOTYPE IN PATIENTS WITH SPORADIC MTC AND PATIENTS WITH HEREDITARY MTC}

\section{[C-1] Sporadic MTC}

Sporadic MTC usually occurs between the fourth and sixth decades of life (33). Central and lateral compartment lymph node metastases are present respectively in $14 \%$ and $11 \%$ of patients with T1 tumors and in $86 \%$ and $93 \%$ of patients with T4 tumors (34). Unfortunately, $70 \%$ of patients with MTC who present with a palpable thyroid nodule have cervical metastases and 10\% have distant metastases (35). On univariate analysis prognosis is directly related to patient age at diagnosis, male sex, the presence of local tumor invasion, the presence of lymph node metastases, and the presence of distant metastases. On multivariate analysis, however, only age and stage of disease at the time of diagnosis are significant independent prognostic factors $(36,37)$. Ten-year survival rates for patients with stages I, II, III, and IV MTC are $100 \%$, $93 \%, 71 \%$, and $21 \%$, respectively (37). The clinical behavior of sporadic MTC is unpredictable; however, some patients with distant metastases may live for several years. In recent decades there has been no significant trend toward an earlier stage of disease at the time of diagnosis, as just under half of the patients present with stage III or IV disease. Also, there has been no significant increase in patient survival $(38,39)$.

\section{[C-2] Hereditary MTC}

In 1968 Steiner and colleagues described a family with the concurrence of MTC, pheochromocytoma (PHEO), hyperparathyroidism (HPTH), and Cushing's syndrome. They suggested that the entity be named multiple endocrine neoplasia type 2 (MEN2) in contradistinction to the previously described hereditary disease, MEN1 $(40,41)$. The syndrome that Steiner and associates described is now known as MEN2A (Online Mendelian Inheritance in Man [OMIM], \#171400, incidence $1 / 1,973,500)(1)$. In retrospect the earliest documented family with MEN2A was from Sweden and concerned a kindred dating to the early 1700s (42). A branch of the Swedish kindred immigrated to the United States and was studied extensively by Tashjian and colleagues (9). As more families were studied, the disease spectrum of MEN2A has expanded to include two variants: patients with associated cutaneous lichen amyloidosis (CLA) and patients with associated $\operatorname{HD}(43,44)$.

The MEN2B syndrome (OMIM \#162300, incidence 1/ $38,750,000)$ variably described by Williams and Pollock, Schimke and colleagues, and Gorlin and associates, accounts for $5 \%$ of hereditary MTCs $(1,45-47)$. Patients with MEN2B develop MTC and PHEOs and exhibit a recognizable phenotype.

Farndon and associates described FMTC [OMIM \#155240] (48). Originally, strict criteria defined the diagnosis of FMTC: more than 10 family members with MTC, multiple carriers or affected members over 50 years of age, and an adequate medical history (particularly in older family members) to exclude the presence of PHEO and HPTH (2). A less rigid def- inition was the presence in at least four family members of MTC without other manifestations of MEN2A (49). Defining and distinguishing FMTC from MEN2A has been challenging, with the controversy focusing on the concern that premature categorization of a family with FMTC could result in failure to identify a PHEO (50). This is illustrated by families with the RET codon G533C mutation in exon 8. In 2003 a large sixgeneration Brazilian family with this mutation, including 76 gene carriers (29 with MTC and none with PHEO or HPTH), was described as having FMTC (51). Also, two Greek families with the RET codon G533C mutation, including 20 carriers, six with MTC and none with PHEO or HPTH, were reported as having FMTC (52). Subsequently, a patient in the Brazilian kindred developed a PHEO, and investigators in Greece and the United States reported additional families with the RET codon G533C mutation who had MTC and PHEO, thus clarifying that this mutation is associated with MEN2A (53-56). At present, there are only three documented families that meet the original strict criteria described for FMTC (2,57-59).

Currently, the opinion of most clinical investigators is that FMTC should not be a freestanding syndrome; rather it should represent a variant along the spectrum of disease expression in MEN2A. The Task Force agrees that FMTC should not be defined as a form of hereditary MTC distinct from MEN2A and MEN2B $(2,49)$. Rather it should be recognized as a variant of MEN2A to include families with only MTC who meet the original strict criteria for FMTC, small families of at least two generations with at least two, but less than 10 , subjects with RET germline mutations, small families in which two or fewer members in a single generation have RET germline mutations, and single individuals with a RET germline mutation (see Supplementary Information).

\section{- RECOMMENDATION 2}

There should be two MEN2 syndromes: MEN2A and MEN2B. Within MEN2A, which accounts for 95\% of MEN2 cases, there should be four variants: classical MEN2A (represented by the uniform presence of MTC and the less frequent occurrence of PHEO, or HPTH, or both), MEN2A with CLA, MEN2A with HD, and FMTC (families or individuals with RET germline mutations who have MTC but neither PHEOs nor HPTH). Grade C Recommendation

\section{[C-2-1] MEN2A}

[C-2-1-1] Classical MEN2A. Classical MEN2A is the most common MEN2A variant and in $95 \%$ of patients RET germline mutations occur in codons $609,611,618$, or 620 of exon 10 or codon 634 of exon 11 (60). Virtually all patients develop MTC and lower numbers develop PHEOs or HPTH, the frequency of each depending on the specific RET mutation. For example RET codon 634 mutations are associated with a high penetrance of PHEO, which in one study increased with age, being $25 \%$ by age 30 years, $52 \%$ by age 50 years, and $88 \%$ by age 77 years (61). There is a much lower penetrance of PHEO in patients with exon 10 RET codon mutations (609 [4\%-26\%], 611 [10\%-25\%], 618 [12\%23\%], and 620 [13\%-24\%]) (62). The PHEOs are almost always benign and are usually multicentric, bilateral, and confined to the adrenal gland. The tumors are usually associated with diffuse nodular adrenal medullary hyperplasia, particularly in patients with RET germline mutations in 
codons 918 and 634 (63). Patients with MEN2A and a unilateral PHEO usually develop a contralateral PHEO within 10 years (63). Prior to the development of biochemical and genetic tests to detect MTC in patients with classical MEN2A, the most common cause of death was PHEO not MTC (64). The HPTH in patients with classical MEN2A is usually mild and associated with few if any symptoms. From one to four parathyroid glands may be enlarged. A RET codon $634 \mathrm{mu}-$ tation is associated with a moderate penetrance of HPTH (up to $30 \%$ ), and RET mutations in codons $609,611,618$, and 620 are associated with a penetrance between $2 \%$ and $12 \%$ $(62,65)$. For practical reasons, screening for HPTH should be done concurrently with screening for PHEO.

There are very rare families with features of classical MEN2A who have no identifiable RET germline mutation. In this situation the diagnosis of classical MEN2A can be made if one or more first-degree relatives have characteristic clinical features of the entity.

[C-2-1-2] MEN2A and CLA. CLA is a rare disorder that usually occurs sporadically but may present in a hereditary pattern, either as a separate entity or in association with other diseases, one of which is MEN2A (66). The CLA in MEN2A is characterized by dermatological lesions that are particularly evident in the scapular region of the back corresponding to dermatomes T2-T6 $(43,67)$. The classic symptom of CLA is intense pruritus that improves with sun exposure and worsens during periods of stress. Hyperpigmented lesions develop later, apparently secondary to scratching. The inciting lesion appears to be notalgia paresthetica, a sensory neuropathy involving the dorsal spinal nerves (66). The CLA may be present at a young age and prior to the onset of clinically evident MTC, thus serving as a precursor for the syndrome $(43,67)$. The CLA in patients with MEN2A occurs almost exclusively in patients with the RET codon $634 \mathrm{mu}-$ tation, although it has been reported in a patient with a codon 804 mutation $(68,69)$. In one study CLA, or regional pruritus without CLA, occurred in $36 \%$ of patients with the RET codon 634 mutation (67). PHEOs and HPTH occur in this variant with the same frequency as in classical MEN2A (67).

Moisturizing lotions and creams, local corticosteroids, systemic antihistamines, and phototherapy usually provide incomplete symptom relief of the pruritus. Three patients with advanced MTC and CLA experienced rapid disappearance of the skin disease in response to treatment with the tyrosine kinase inhibitor (TKI), vandetanib; however, the CLA reappeared following dose reduction (M. Schlumberger and R. Gagel; personal communication, May 2008 and June 2009).

[C-2-1-3] MEN2A and HD. RET germline mutations are present in $50 \%$ of patients with hereditary HD and in $15 \%-$ $20 \%$ of patients with sporadic HD (30). Over 100 RET mutations have been described in HD, including microdeletions and insertions, nonsense or missense point mutations, splicing mutations, or deletions encompassing segments of the $R E T$ gene (31). The RET mutations in patients with MEN2A and HD are point mutations involving codons in exon 10: 609 (15\%), $611(5 \%), 618(30 \%)$, and $620(50 \%)(70,71)$. HD occurs in approximately $7 \%$ of patients with MEN2A $(72,73)$. Conversely, $2 \%-5 \%$ of patients with HD have MEN2A $(73,74)$. HD is almost always apparent shortly after birth; however, it is important to exclude HD in older patients with
MEN2A and exon 10 RET mutations who have colon symptoms suggestive of HD. Conversely, patients with HD who have exon 10 RET mutations should be evaluated for MEN2A.

It seems paradoxical that MEN2A and HD should occur together, since the RET mutations associated with HD are "loss of function," while the RET mutations associated with MEN2A are "gain of function." The generally accepted explanation for this dual occurrence is that constitutive activation of RET is sufficient to trigger neoplastic transformation of the C-cells and adrenal chromaffin cells, yet insufficient to generate a trophic response in the precursor neurons due to a lack of expression of the RET protein at the cell surface (75).

[C-2-1-4] FMTC. FMTC is characterized by the presence of a $R E T$ germline mutation in families with MTC (or single individuals with MTC and no family history of MTC) who develop neither PHEOs nor HPTH.

\section{[C-2-2] MEN2B}

In patients with MEN2B the MTC often presents in infancy and is highly aggressive, metastasizing early to regional lymph nodes and beyond. Approximately $75 \%$ of MEN2B cases are sporadic and affected patients have de novo RET mutations, while $25 \%$ of cases occur in families with previous or current manifestations of MEN2B. Approximately $95 \%$ of patients with MEN2B have RET germline mutations in exon 16 (codon $\mathrm{M} 918 \mathrm{~T}$ ) and fewer than 5\% have RET germline mutations in exon 15 (codon A883F) (16,18,76,77). Almost all published reports on MEN2B concern patients with the RET codon M918T mutation and little is known about the clinical behavior of patients with the RET codon $A 883 F$ mutation. Recent reports, however, suggest that patients with the A883F codon mutation have a less aggressive MTC compared to patients with the M918T codon mutation (78).

Approximately $50 \%$ of patients with MEN2B develop PHEOs. They also have a unique physical appearance characterized by a typical facies, ophthalmologic abnormalities (inability to make tears in infancy, thickened and everted eyelids, mild ptosis, and prominent corneal nerves), skeletal malformations (marfanoid body habitus, narrow long facies, pes cavus, pectus excavatum, high-arched palate, scoliosis, and slipped capital femoral epiphyses), and a generalized ganglioneuromatosis throughout the aerodigestive tract. Most patients have abdominal symptoms characterized by bloating, intermittent constipation, and diarrhea, and some patients require surgery for intestinal obstruction $(79,80)$. It is important to establish the diagnosis of MEN2B at an early age when there is a possibility that thyroidectomy will be curative. However, the reality is that most patients with MEN2B are diagnosed when the MTC is clinically evident and too advanced to be cured (81).

A rare group of patients have atypical MEN2B that develops around 20 to 30 years of age. The patients have double $R E T$ germline mutations appearing in tandem on the same allele and involving RET codon V804M and either RET codon Y806C, S904C, E805K, or Q781R (82-85). Evaluation of the tandem mutations by in vitro and in silico analysis provides information about their transforming ability (prediction scores). Using this methodology, each of the four reported double RET mutations had high transforming ability compared to the single mutations of the pairs, supporting the presence of a more aggressive MTC. Similar double RET germline mutations have been identified in families with the FMTC variant of MEN2A $(86,87)$. 


\section{[D] DIRECT DNA ANALYSIS TO DETECT MUTATIONS IN THE RET PROTOONCOGENE}

A sequence change in the RET gene is considered to be a causative MEN2 mutation if it segregates with the clinical expression of disease within a family including at least two affected individuals having the MEN2A or MEN2B phenotype. In contrast, benign germline RET sequence changes not causative of MEN2 are considered polymorphisms; for example, $\mathrm{p}$. G691S, p. L769L, p. S836S, p. S904S, or intron 14 c.2608$24 \mathrm{G}>\mathrm{A}$. If insufficient clinical information is available (e.g., only one gene carrier with disease or incidentally detected RET sequence changes without disease manifestations) a preliminary classification of "variant of unknown significance" is suggested. Classification of risk groups in patients with MEN2A, based on aggressiveness of MTC or penetrance estimates of PHEO or HPTH, is only meaningful if adequate numbers of patients are available for valid risk assessment.

Currently, more than 600 international laboratories offer molecular genetic testing, biochemical testing, and cytogenetic testing for more than 3000 inherited disorders (88). Many of the laboratories offer prenatal diagnosis. Screening for hereditary MTC is relatively simple when one knows the $R E T$ germline mutation carried in the family because a targeted approach can detect the specific mutated RET allele in at-risk family members. In new families with hereditary MTC, in whom the specific RET mutation is unknown, the usual strategy is to sequence initially the most commonly mutated $R E T$ cysteine codons in exons 10 and 11 (C609, C611, C618, C620, C630, and C634) as well as additional RET codon mutations in exons 13, 14, 15, and 16. A few laboratories include exon 8 (89). Some laboratories sequence the entire RET coding region, although the cost increases as the extent of sequencing increases. The expense of sequencing has decreased markedly over the last few years and will continue to do so, such that soon it may be relatively inexpensive to sequence the entire $R E T$ coding region as the first step in screening. Other laboratories use a two-tiered approach, starting with analysis of the most commonly mutated "hotspot" exons. At the request of the ordering physician, the laboratories sequence the remaining $R E T$ exons if the initial analysis is negative or if the patient's phenotype is inconsistent with the RET mutation initially identified. However, tiered approaches may fail to detect rare $R E T$ double or multiple mutations that would be identified by sequencing the entire RET coding sequence. For example, as already mentioned, codon 804 mutations occur in conjunction with a second RET mutation in rare cases of MEN2B or the FMTC variant of MEN2A. Also, in rare patients with MEN2A, there are double RET mutations or even triple or quadruple $R E T$ mutations that target residues other than codon V804 (89-93). The occurrence of multiple RET mutations may cause an unusual clinical phenotype compared to that seen with the corresponding single RET mutations. This situation has been reported recently in five kindreds from Brazil featuring coincident Y791F and C634Y RET codon mutations $(94,95)$.

\section{- RECOMMENDATION 3}

The recommended method of initial testing for MEN2A is either a single or multi-tiered analysis to detect RET mutations in exon 10 (codons 609, 611, 618, and 620), exon 11 (codons 630 and 634), and exons 8, 13, 14, 15, and 16. Grade B Recommendation

\section{- RECOMMENDATION 4}

Sequencing of the entire coding region should be reserved for situations in which no RET mutation is identified or there is a discrepancy between the MEN2 phenotype and the expected genotype. Grade B Recommendation

\section{- RECOMMENDATION 5}

Patients with the MEN2B phenotype should be tested for the RET codon M918T mutation (exon 16), and if negative, the $R E T$ codon $\mathrm{A} 883 \mathrm{~F}$ mutation (exon 15). If there are no mutations identified in these two exons the entire RET coding region should be sequenced. Grade B Recommendation

\section{[D-1] Direct DNA analysis to detect RET germline mutations in patients with apparent sporadic MTC}

Importantly, $1 \%-7 \%$ of patients with presumed sporadic MTC actually have hereditary disease $(96,97)$. Patients with sporadic MTC should have genetic counseling and direct DNA analysis to detect a mutated $R E T$ allele. Patients found to have a RET germline mutation should be evaluated, and their firstdegree relatives should be offered genetic counseling and genetic testing. Genetic counseling and genetic testing should also be considered in populations at high risk for hereditary MTC, such as patients with CLA and patients with HD who have exon 10 mutations. In 5\%-9\% of patients with MEN2A, and the large majority of patients with MEN2B, the RET mutation arises de novo and almost always from the paternal allele $(98,99)$. At present there is no indication for evaluating the thyroid tumors of patients with sporadic MTC for the presence of somatic HRAS, $K R A S$, or NRAS mutations, or the RET codon M918T mutation.

\section{- RECOMMENDATION 6}

Patients with presumed sporadic MTC should have genetic testing to detect a germline RET mutation. If a RET mutation is found the patient should have genetic testing. Grade B Recommendation

\section{- RECOMMENDATION 7}

Genetic counseling and genetic testing for RET germline mutations should be offered to

a) first-degree relatives of patients with proven hereditary MTC,

b) parents whose infants or young children have the classic phenotype of MEN2B,

c) patients with CLA, and

d) infants or young children with HD and exon $10 R E T$ germline mutations, and adults with MEN2A and exon 10 mutations who have symptoms suggestive of HD.

Grade B Recommendation

\section{- RECOMMENDATION 8}

Other than for academic reasons or physician preference, it is not standard practice to analyze tumors of patients with sporadic MTC for somatic HRAS, KRAS, or NRAS mutations, or the RET M918T mutation. Grade C Recommendation

\section{- RECOMMENDATION 9}

In very rare families who meet the clinical criteria for MEN2A or 2B, despite negative sequencing of the entire RET coding region, the relatives at risk should be periodically 
screened by conventional methods for MTC, PHEO, and HPTH. After the initial evaluation, screening should continue at 1- to 3-year intervals. Grade C Recommendation

\section{[E] ETHICAL CONSIDERATIONS FOR GENETIC SCREENING}

\section{[E-1] Adults}

In a physician-patient relationship the legal and ethical duty to warn identifiable third parties of foreseeable harm was established in Tarasoff $v$. Regents of the University of California in which the court held that "privacy ends where the public peril begins" (100). In Tarasoff, the failure to warn a woman about premeditated homicide by her boyfriend, who had confided the plan to his University of California psychologist, led to a new standard for warning third parties who wittingly or unwittingly may be an "agent of harm." The Tarasoff ruling is a translational health law precedent that has been widely applied to numerous clinical contexts.

In the Pate v. Threlkel case, a surgeon was found negligent for failing to warn his patient that her children were at risk for hereditary MTC (101). The court held: "Physicians may owe a legal duty to the children of a patient when they are identified beneficiaries of the prevailing standard of care." This ruling reinforced the identification of "at-risk" third parties and defined boundaries for physicians in determining who should be warned (102).

In Safer v. the Estate of Pack, another case assessing duty to warn in a family with familial polyposis syndrome, it was established that physicians could not satisfy the duty to warn merely by informing the patient of possible risks (103). They must take "reasonable steps to guarantee that immediate family members are warned" (104). Given the complexity of the information, and what is known about occult psychosocial barriers to screening, it should not be expected that patients are qualified to explain the genetic aspects of their disease or to discuss potential therapies to family members when they lack the scientific or medical competence to do so. The patient, however, needs to participate in identifying at-risk relatives for possible screening. An ethical dilemma arises only when the patient refuses permission to contact at-risk relatives or states that he does not wish to disclose his health information to family members. In this context, it is usually permissible ethically, or is even obligatory, to warn third parties without the patient's consent. In such a dilemma a case-based approach is needed with involvement of clinical ethicists, social workers, and legal counsel. There are proposed criteria for evaluating the permissibility of duty to warn, which include gravity, probability, imminence of harm, identifiability of at-risk individuals, probability that intervention can mitigate harm, the degree to which means of maintaining confidentiality have been exhausted, and whether the patient is an agent of harm. Most cases of genetic risk in cancer do not satisfy all of these criteria; however, hereditary MTC satisfies each of them $(102,104)$.

There is no clear guidance from professional societies on the matter of informing family members at risk when the affected relative is reluctant to do so. The American Medical Association and the American Society of Clinical Oncology recommend restricting initial disclosure of test results to the person who had the test, but encourage working with the patient to identify and notify at-risk family members $(105,106)$. On the other hand, The American Society of Human Genetics supports disclosure of genetic information under exceptional circumstances to at-risk family members, even though it might be inconsistent with the wishes of the patient (107). On a somewhat different matter, the American College of Medical Genetics states that disclosure of RET mutations should be mandatory when found incidentally during exom and whole genome sequencing (108). Earlier literature on HIPAA is less clear about whether breaching HIPAA in the MTC context places the physician at risk $(109,110)$.

\section{- RECOMMENDATION 10}

Regarding hereditary MTC, the duty to warn a competent and capacitated patient or surrogate decision maker of the risk that an inherited RET mutation may pose to family members is a standard of care. This warning is ideally fulfilled in the setting of genetic counseling and should include a request for the patient to participate in identifying at-risk relatives. The "duty to warn" discussion should be a part of the informed consent process, in which there is full disclosure of the seriousness of the disease and available forms of prevention and treatment. When a patient refuses to notify relatives or legal dependents of their risks, the physician should consider whether he has an ethical duty or obligation to warn family members at risk. He should consult a trained clinical ethicist either at his medical center or another medical facility or contact the American Thyroid Association Ethics Committee for guidance. Grade A Recommendation

\section{[E-2] The pediatric population}

In the pediatric population the concern lies in identifying children at risk for inheriting MEN2 from a parent who refuses to disclose to the child their specific risks and the available preventative or therapeutic options. It may be necessary to involve state officials and the courts to resolve such issues in order to protect the child. Regarding informed consent in a pediatric context, physicians should refer to published statements by professional organizations and clinical ethicists: Pediatric Ethics Guidelines for Inherited Medullary Thyroid Cancer and the American Pediatric Association Committee on Bioethics statement: Informed Consent, Parental Permission, and Assent in Pediatric Practice $(111,112)$.

\section{- RECOMMENDATION 11}

With pediatric patients who have not reached the age of consent, it may be necessary for physicians to seek state intervention to prevent harm when there is parental refusal to inform their children of the risk of developing a malignant tumor. Practitioners with pediatric populations should consult published documents for guidance. Grade A Recommendation

\section{[E-3] Reproductive options of RET mutation carriers}

Prenatal and pre-implantation testing is available to individuals with MEN2 (113-116). Prenatal testing can be performed in the first or second trimester by chorionic villus sampling or amniocentesis, respectively. Also, it is possible to perform prenatal testing on fetal blood cells obtained from maternal blood (117). High-resolution melting genotype analysis can detect RET mutations in the serum of patients 
with MEN2A. Recently, the technique was applied prenatally to detect a RET codon $C 634 Y$ mutation in a fetus by identifying the mutation in blood from the unaffected pregnant mother whose husband had a C634Y codon mutation (118). Such testing might be offered to couples in which only the father has hereditary MTC and the parents wish to know the mutational status of the fetus.

Pre-implantation genetic diagnosis (PGD) is an in vitro fertilization technique that isolates embryonic cells for singlesite RET testing. The procedure involves removal of one or two cells from an eight-cell blastocyst followed by sequence analysis of the DNA of the removed cells to determine if a $R E T$ mutation is present. Nonmutated embryos are then implanted into the mother, providing the opportunity to eliminate the disease gene from the family (119). Successful delivery of an unaffected child from an affected mother has been achieved with this technique and advances with the technology have led to higher pregnancy rates $(120,121)$.

The role of PGD in adult-onset disease remains controversial. It is generally offered to patients with syndromes characterized by a young age of onset with significant cancer risk and associated morbidity or mortality. With an average age of MTC onset well under 30 years of age-much lower in patients with MEN2B - and a risk of PHEO up to 50\%, PGD may be an option for parents if one of them has MEN2 (especially those with RET mutations in codons 634 and 918). In practice, however, there is little evidence that clinicians or patients have availed themselves of this technology over the past decade, despite its widespread use for other genetic disorders. Two possible reasons may explain the lack of participation. First is the perception that the MTC in youngsters with MEN2A can be managed by timely surgical intervention. There are children with MEN2A who have been treated by prophylactic thyroidectomy and are alive without evidence of recurrent MTC decades later. Second, it can be very difficult to convince young parents to spend a substantial amount of money for PGD and in vitro fertilization (usually not covered by insurance), when there is the alternative of managing the individual manifestations of the disease (usually covered by health insurance) if and when they occur. Nonetheless, it is important for the physician to make patients aware of this technology or refer them to a genetic counselor with expertise in this area. There should be appropriate documentation of the exchange of information.

The surgical management of children with MEN2B is challenging, and few are cured. Therefore, young parents (one of whom has MEN2B) planning to have children should be informed of the availability of in vitro fertilization and PGD.

\section{[E-4] Ethical considerations for preconception and prenatal counseling}

Preconception counseling and prenatal testing are available to individuals with hereditary MTC. While a patient may not wish to proceed with prenatal pre-implantation diagnosis, the clinician has both a legal and ethical duty to warn in the prenatal context as well. The courts held in Molloy v. Meier, a case concerning Fragile $\mathrm{X}$ syndrome, that physicians have a duty to warn of genetic harms and to inform patients of all available prenatal medically appropriate options (122). Failure to warn in a prenatal context could lead to allegations of wrongful conception, wrongful life, or even wrongful birth, in which case the parents or the offspring could sue the physician for damages related to a foreseeable and serious genetic disease or condition.

Parents who do not wish to have prenatal RET mutation testing should be offered genetic counseling and genetic testing of their child, the timing to be determined by the treating physician in consultation with the child's parents (112). The foregoing statement assumes that the fetus is a patient. Fetuses are not always patients if the mother does not intend to complete the pregnancy.

\section{- RECOMMENDATION 12}

The duty to warn of genetic risk extends to both preconception and prenatal contexts. Genetic counseling about the options of pre-implantation or prenatal diagnostic testing should be considered for all RET mutation carriers of childbearing age, particularly those with MEN2B. Parents who do not wish to have prenatal RET mutation testing should be offered genetic counseling and informed of the availability of genetic testing of their child to detect a mutated RET allele. This is particularly important for mutations associated with the onset of MTC before 5 years of age. Grade A Recommendation

\section{[F] SECRETORY PRODUCTS OF MTC}

The C-cells of the thyroid gland secrete several hormones or biogenic amines, including adrenocorticotropic hormone $(\mathrm{ACTH}), \mathrm{B}-\mathrm{melanocyte}$ stimulating hormone, Ctn, carcinoembryonic antigen (CEA), chromogranin, histaminase, neurotensin, and somatostatin (123-128). Of these secretory products, Ctn and CEA are valuable tumor markers in patients with MTC, and their serum concentrations are directly related to the C-cell mass.

\section{[F-1] Calcitonin}

Calcitonin is a 32-amino acid monomeric peptide that results from cleavage and post translational processing of procalcitonin, a precursor peptide derived from preprocalcitonin (129). Over the past decade commercial assays for measuring Ctn have progressed to the newest immunochemiluminometric assays (ICMAs) that are highly sensitive and specific for monomeric Ctn. With ICMAs, cross-reactivity with procalcitonin or other calcitonin-related peptides is largely eliminated. This is important because sepsis or other general inflammatory conditions may cause profound elevations of procalcitonin in tissues that do not normally transcribe the Ctn gene $(130,131)$. Serum Ctn levels may be increased in patients with chronic renal failure, and other ailments, such as HPTH, autoimmune thyroiditis, small cell and large cell lung cancers, prostate cancer, mastocytosis, and various enteric and pulmonary neuroendocrine tumors (132-139). The serum Ctn levels in patients with the various nonthyroid malignancies do not increase in response to calcium or pentagastrin stimulation, and compared to MTC, the tumors usually produce less Ctn per gram of tissue.

Heterophilic antibodies (human antibodies with a broad reactivity with antibodies of other animal species) cause falsely elevated (and rarely falsely lower) serum Ctn levels (140). The "hook effect" refers to the detection of falsely low analyte levels in the immunoassay resulting from very high serum levels of Ctn (as occurs in patients with widely disseminated MTC), which saturate the binding capacity of the antibody (141). The hook effect is less likely to occur with 
ICMAs, but should remain a concern in patients with a large tumor burden and surprisingly low serum Ctn levels.

Depending on the assay, $56 \%-88 \%$ of normal subjects have serum Ctn levels below the functional sensitivity, while 3\%$10 \%$ have Ctn levels greater than $10 \mathrm{pg} / \mathrm{mL}$ (142). Current reference ranges for serum $\mathrm{Ctn}$ are higher in men compared with women, almost certainly due to the larger C-cell mass in men (143,144). Using the Advantage system (Nichols Institute Diagnostics, San Juan Capistrano, CA), Basuyau and associates found the 95th percentile for serum Ctn levels to be $5.2 \mathrm{ng} / \mathrm{L}$ in women and $11.7 \mathrm{ng} / \mathrm{L}$ in men (143). There may be variability in Ctn measurements among commercial assays, emphasizing the imperative of evaluating individual patient samples in the same assay before and after thyroidectomy and during treatment for advanced disease. Laboratories should notify clinicians when there is a change in assay methodology, including the reference ranges of the new Ctn assay. The current revised MTC guidelines do not specify reference ranges of basal or stimulated serum Ctn levels. Based on studies of large numbers of normal patients and patients with MTC, individual laboratories may set their own criteria defining reference ranges for basal and elevated serum Ctn levels.

In studies evaluating serum levels of $\mathrm{Ctn}$ and procalcitonin in patients with MTC, Ctn was found to have equal or superior diagnostic accuracy. However, a high procalcitonin to $\mathrm{Ctn}$ ratio was correlated with an increased risk of progressive disease and a shortened progression-free survival (PFS), potentially useful in predicting prognosis $(145,146)$. Although of interest, physicians rarely use serum procalcitonin levels in the management of patients with MTC.

There are sparse data on serum Ctn levels in young children. Previous studies have shown that serum Ctn concentrations are particularly high during the first week of life and in lowbirthweight children and premature infants (143). Basuyau and colleagues proposed a reference range of less than $40 \mathrm{pg} / \mathrm{mL}$ in children less than 6 months of age and less than $15 \mathrm{pg} / \mathrm{mL}$ in children between 6 months and 3 years of age. The highest serum Ctn value observed in their series was $75 \mathrm{pg} / \mathrm{mL}$ in a child age 4.5 months. A follow-up value 1 month later was $32.4 \mathrm{pg} / \mathrm{mL}$ (143). Serum Ctn values in children more than 3 years of age were indistinguishable from those observed in adults.

Administrating potent secretagogues, such as intravenous calcium or pentagastrin, may increase the sensitivity of $\mathrm{Ctn}$ testing. There are mild but unpleasant side effects associated with the infusion of these agents, although they are less bothersome with calcium. In some studies pentagastrin was a more potent secretagogue than calcium, while in other studies the converse was true (147-150). Some clinical investigators feel that the sensitivity of the ICMA is such that provocative testing is no longer necessary; however, others consider provocative testing useful in determining the timing of thyroidectomy in children who have inherited a mutated RET allele, in the evaluation of patients for persistent or recurrent MTC following thyroidectomy, and for detecting MTC in patients with nodular goiters $(148,150)$. It is critical that clinicians who use provocative testing establish clear reference guidelines for abnormal serum Ctn levels in their clinics, a more challenging task than establishing reference guidelines for abnormal basal Ctn levels.

\section{- RECOMMENDATION 13}

Clinicians should be aware that falsely high or low serum Ctn levels might occur with a variety of clinical diseases other than MTC. They should consider this possibility when serum Ctn levels are disproportionate to the expected clinical findings. Grade C Recommendation

\section{- RECOMMENDATION 14}

In interpreting serum Ctn data, clinicians should be aware that $\mathrm{Ctn}$ levels are markedly elevated in children under 3 years of age, especially under 6 months of age. Also, Ctn levels are higher in males compared with females. Grade B Recommendation

\section{[F-2] Carcinoembryonic antigen}

CEA is not a specific biomarker for MTC. Primarily for this reason, and also because serum CEA levels do not increase following calcium or pentagastrin stimulation, measurement of this tumor marker is not useful in the early diagnosis of MTC. Determination of serum CEA levels is useful for evaluating disease progression in patients with clinically evident MTC and for monitoring patients following thyroidectomy (151).

False elevation of serum CEA may result from heterophilic antibodies, smoking tobacco, or other conditions, such as gastrointestinal tract inflammatory disease, benign lung disease, or a host of nonthyroid malignancies. In patients with MTC simultaneous elevations of serum CEA and Ctn levels indicate disease progression. Some patients with progressive disease have increasing serum CEA levels associated with stable or declining serum Ctn levels. This is considered an indication of poorly differentiated MTC and is supported by CEA and Ctn immunohistochemistry findings (152). In one study, CEA and Ctn were expressed in almost every MTC cell. This was especially true for C-cell hyperplasia $(\mathrm{CCH})$, microscopic MTC, and gross MTC confined to the thyroid. By contrast, there was an inverse relationship between CEA and Ctn staining in the tumors of patients with invasive or metastatic MTC. The most aggressive tumors had persistent and intense CEA staining, but minimal if any $\mathrm{Ctn}$ staining. It was suggested that CEA is a marker for early epithelial differentiation and therefore retained, while Ctn is a late marker for terminal differentiation and therefore lost (152).

Rarely, patients with advanced MTC have normal or low serum levels of $\mathrm{Ctn}$ and CEA. This unusual state represents either a misdiagnosis or advanced dedifferentiation of the MTC, in which case it conveys a poor prognosis $(153,154)$. In a study of 839 patients with sporadic MTC, seven $(0.83 \%)$ with advanced disease secreted neither Ctn nor CEA (155). Poorly differentiated histology, a high Ki-67 proliferation index (three patients), and a high proportion of RET codon M918T mutated cells (four patients) characterized the aggressive biological behavior of the MTC. The prognosis of the patients with nonsecretory MTC varied between intermediateterm survival (12.5 years) and rapid progression leading to death within 1.75 years after diagnosis (155).

\section{- RECOMMENDATION 15}

Basal levels of serum Ctn and CEA should be measured concurrently. In patients with advanced MTC, a marked elevation in the serum CEA level out of proportion to a lower serum Ctn level or normal or low levels of both serum Ctn and CEA indicate poorly differentiated MTC. Grade B Recommendation 
[G] MORPHOLOGICAL EXAMINATION OF AN EXCISIONAL BIOPSY OR THE THYROIDECTOMYSPECIMEN

As the ultimobranchial bodies migrate from the neural crest during embryogenesis, they become entrapped within the middle and upper poles of each thyroid lobe. There, they give rise to $\mathrm{C}$-cells, which are much more numerous in males compared with females (144). Unlike other thyroid tumors, which arise from follicular cells, MTC is a neuroendocrine tumor. It is classified as a thyroid tumor because of its anatomical location.

Macroscopically, MTC is firm and either white, tan, or red in color. Sporadic MTC usually occurs as a solitary unilateral tumor, whereas hereditary MTC is usually multicentric and bilateral. On histological examination the MTC cells are typically round, polyhedral, or spindle shaped and form sheets or nests with peripheral palisading in a vascular stroma. The amyloid material present in over half of MTCs is actually composed of full-length Ctn (156).

There may be substantial variance in the histological appearance of MTC, such that it can be confused with PTC, follicular thyroid carcinoma (FTC), paraganglioma, and even lymphoma or sarcoma. Oncocytic change occasionally occurs in these tumors, further confounding the diagnosis. The MTC cells express cytokeratins, mainly CK7 and CK18, NKX2.1 (TTF1), and chromogranin A, but the most important diagnostic markers are $\mathrm{Ctn}$ and $\mathrm{CEA}$.

Immunohistochemical (IHC) staining of Ctn may vary in intensity and extent, but in its absence a diagnosis of MTC should be questioned. The IHC reactivity of Ctn is often reduced in undifferentiated tumors, whereas staining for CEA is almost always strongly positive (152). The College of American Pathologists has recommended synoptic reporting as a mechanism to ensure consistent and complete documentation of cancer pathology. The synoptic data recommended for the evaluation of MTC are provided in the Protocol for the Examination of Specimens from $\mathrm{Pa}$ tients with Carcinomas of the Thyroid Gland (available at www.cap.org).

Occasionally, PTC or FTC occurs concurrently with MTC, and although the relationship of the two components of the complex tumors is controversial, their coexistence is thought to represent a coincidental collision with intermingling of neoplastic C-cells and follicular cells $(157,158)$. A recent study from Germany identified simultaneous MTC and PTC in $26(2.6 \%)$ of 1019 PTCs, in $6(2.6 \%)$ of 235 hereditary MTCs, and in 20 (4.1\%) of 492 sporadic MTCs (159). The rate of simultaneous MTC and PTC (3.6\%) in the 727 German patients with sporadic and hereditary MTC was much lower than that found in comparable studies from Italy $(13.8 \%$ of 196 patients) and Korea (19\% of 53 patients) $(160,161)$. The reasons for the discrepancies are unknown but may be due to environmental conditions or differences in the study populations. Rarely, the MTC and the follicular component metastasize together as histologically separate tumors in the same target organ (162). Surgery is the primary treatment for patients with MTC mixed with follicular elements. The physician should consider treatment with radioactive iodine (RAI) in the rare situation in which the mixed components or the follicular element alone spreads to lymph nodes or distant sites $(163,164)$.
In hereditary MTC the entity $\mathrm{CCH}$ precedes the development of MTC and is best demonstrated by IHC staining with Ctn antibodies (165). CCH is actually a misnomer. Considering its multicentricity, the entity most likely represents clonal proliferation of multiple transformed progenitor Ccells. Therefore, terms such as C-cell carcinoma in situ or $\mathrm{C}$-cell neoplasia are more appropriate. The criteria for the diagnosis of $\mathrm{CCH}$ are controversial; however, most pathologists agree that a diagnosis is warranted if there are more than seven C-cells per cluster, complete follicles surrounded by $\mathrm{C}$-cells, and distribution of $\mathrm{C}$-cells beyond the normal anatomical location (166). C-cells are normally restricted to the junction of the upper third and lower two-thirds of the lateral lobes; extension beyond this region is considered indicative of an abnormal C-cell proliferation. Atypical C-cell proliferation that breaches the basement membrane or invades the surrounding stroma has the potential to metastasize. Also, the presence of a desmoplastic stromal response indicates an invasive lesion. Patients with presumed sporadic MTC who have CCH or multifocal hyperplasia on morphological examination of the entire gland, should be evaluated for the presence of RET germline mutations. The $\mathrm{CCH}$ that occurs secondarily in association with hyperparathyroidism, chronic lymphocytic thyroiditis, renal insufficiency, and aging is not a premalignant condition (167-170).

The distinction of $\mathrm{CCH}$ from microcarcinoma $(\mathrm{MTC}<1$ $\mathrm{cm}$ and without capsular invasion) is challenging but clinically significant. In a study of 776 patients with MTC, 223 $(30 \%)$ of whom had microcarcinomas, increasing tumor size (from $<2 \mathrm{~mm}$ to $9-10 \mathrm{~mm}$ ) was associated with an increasing incidence of lymph node metastases in patients with hereditary MTC (from 6\% to 62\%; cure rates from $96 \%$ to $71 \%$ ), compared to patients with sporadic MTC (13\% to $43 \%$; cure rates from $85 \%$ to $77 \%$ ). Considering all cases of medullary thyroid microcarcinoma, however, the incidence of distant metastases was only $1.3 \%$ (171).

\section{- RECOMMENDATION 16}

The assessment of a thyroid tumor with any feature suggestive of MTC should include IHC analysis to determine the presence of markers such as Ctn, chromogranin, and CEA and the absence of thyroglobulin. Grade B Recommendation

\section{- RECOMMENDATION 17}

Complete notation of the features of every MTC should follow the synoptic reporting guidelines of the College of American Pathologists Protocol for the Examination of Specimens from Patients with Carcinomas of the Thyroid Gland. Grade B Recommendation

\section{- RECOMMENDATION 18}

In patients with MTC, morphological examination of the entire gland is recommended to determine the presence of $\mathrm{CCH}$ or multifocal neoplasia. Grade C Recommendation

\section{[H] THE DIAGNOSIS OF MTC IN PATIENTS PRESENTING WITH A THYROID NODULE}

\section{[H-1] Fine-needle aspiration biopsy (Figure 1)}

Fine-needle aspiration (FNA) biopsy of thyroid nodules is a useful and safe tool in the diagnosis of thyroid pathology. 


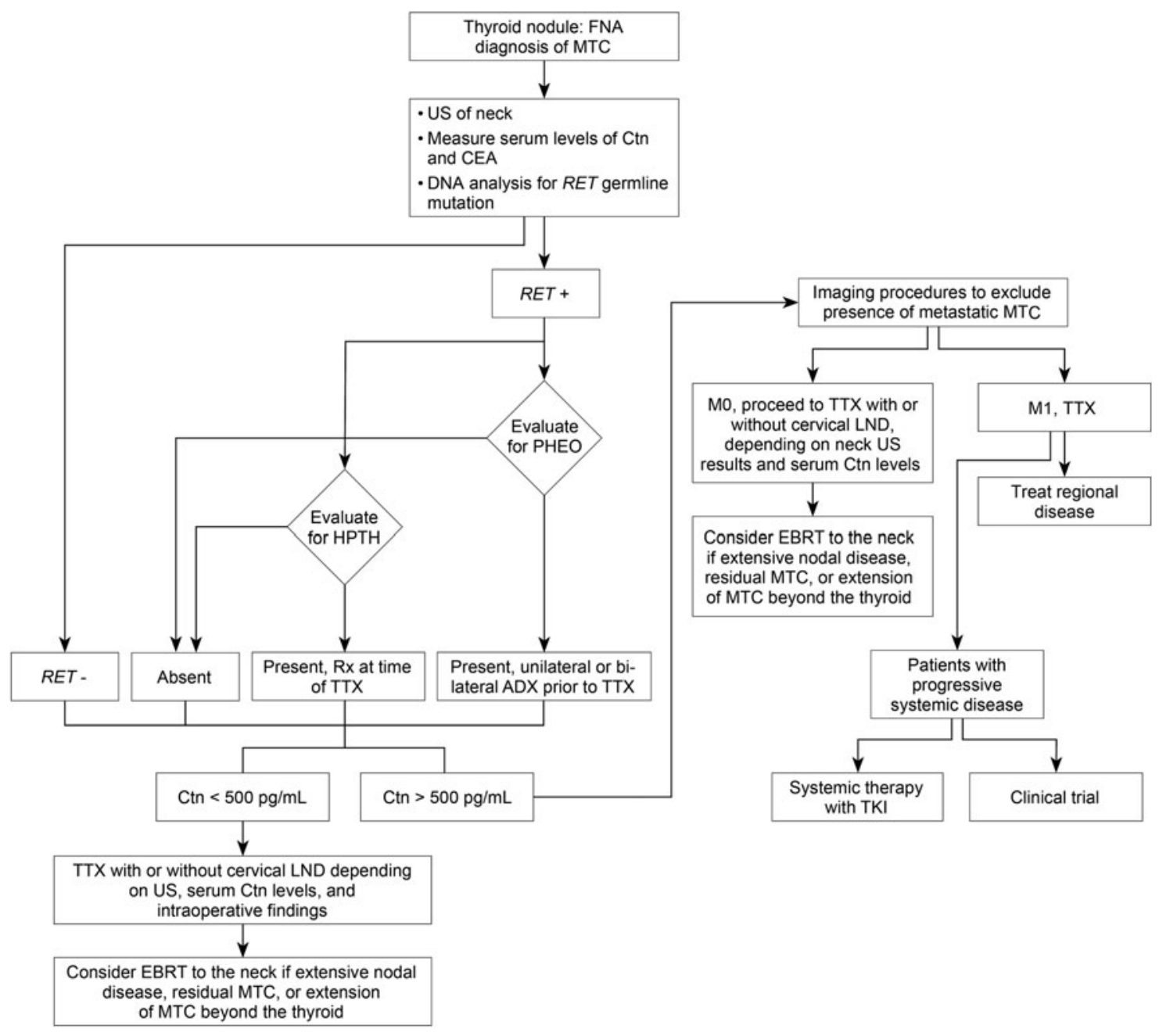

FIG. 1. Management of patients with a thyroid nodule and histological diagnosis of medullary thyroid carcinoma. ADX, adrenalectomy; Ctn, calcitonin; CEA, carcinoembryonic antigen; EBRT, external beam radiotherapy; FNA, fine-needle aspiration; HPTH, hyperparathyroidism; LND, lymph node dissection; MTC, medullary thyroid carcinoma; M, metastatic MTC; PHEO, pheochromocytoma; RET, REarranged during Transfection; TKI, tyrosine kinase inhibitor; TTX, total thyroidectomy; US, ultrasound.

Medullary carcinoma has a variable appearance on aspiration cytology. The MTC cells are usually discohesive or weakly cohesive and may be spindle-shaped, plasmacytoid, or epithelioid. Epithelioid tumors can mimic thyroid follicular lesions, plasmacytoid tumors can be misdiagnosed as plasmacytomas, and pure spindle cell tumors can be mistaken for sarcomas. There may also be bizarre giant cells, oncocytic cells, clear cells, and cells with a small cell carcinoma-like appearance. The tumor cells may contain azurophilic perinuclear cytoplasmic granules. The eccentric nuclei exhibit chromatin granularity as a "salt and pepper" appearance that is typical of neuroendocrine tumors. Amyloid can be mistaken for colloid and on its own is not diagnostic since it may also be present in systemic amyloidosis, amyloid goiter or follicular lesions (172). The diagnosis of MTC can be verified by immunolocalization of $\mathrm{Ctn}$, chromogranin, or CEA; by confirming lack of thyroglobulin staining; and most importantly by detecting elevated serum Ctn levels in the patient.

To investigate pitfalls in the diagnosis of MTC by FNA, cytomorphology was reviewed in the histological slides of 34 patients with MTC. The diagnosis of MTC was correct in
28 cases; however, six cases were misdiagnosed: three as a follicular neoplasm, one as a desmoid tumor, and two as tumors suspicious for MTC. With no benign findings, FNA indicated the need for surgery in all 34 patients (173). Similarly, in a study of 91 cases, FNA findings were diagnostic of MTC in $89 \%$ of cases and indicated the need for surgery in 99\% (174). The most important cytological criteria of MTC were a dispersed cell pattern of polygonal or triangular cells, azurophilic cytoplasmic granules, and eccentrically placed nuclei with coarse granular chromatin and amyloid (174). Although in a meta-analysis of 15 publications, the accuracy of FNA in diagnosing MTC in patients with MTC nodules was less than $50 \%$, IHC analysis of the FNA specimen and measurement of $\mathrm{Ctn}$ in the FNA aspirate were performed rarely (175). The diagnostic accuracy of FNA analysis has been reported to be markedly increased by IHC analysis of the FNA specimen and additionally by measuring Ctn levels in the FNA washout fluid (176). Therefore, FNA should be performed on thyroid nodules that are greater than $1 \mathrm{~cm}$ in size, depending on the ultrasound characteristics. When histological analysis is inconclusive or shows features suggestive of MTC, 
Ctn levels should be measured in the FNA washout fluid, and the specimen should be evaluated by IHC staining to determine the presence of markers such as Ctn, chromogranin, and CEA and the absence of thyroglobulin.

\section{- RECOMMENDATION 19}

Thyroid nodules that are $1 \mathrm{~cm}$ or greater in size should be evaluated by FNA depending on the ultrasound characteristics. FNA findings that are inconclusive or suggestive of MTC should have calcitonin measured in the FNA washout fluid and IHC staining of the FNA sample to detect the presence of markers such as Ctn, chromogranin, and CEA and the absence of thyroglobulin. Grade B Recommendation.

\section{[H-2] Measurement of the serum Ctn in patients with nodular thyroid disease level}

In a recent study investigators measured serum Ctn levels in 10,864 patients with nodular thyroid disease and detected MTC in $0.40 \%$ (177). In this and other studies, serum Ctn measurements had a higher diagnostic sensitivity and specificity compared with FNA findings (177-179). In the largest study of patients diagnosed by Ctn screening, compared to an unmatched historical control group with no Ctn screening, the MTC was present at an earlier disease stage and postoperative serum Ctn levels were more often undetectable. Also, at the end of the follow-up period, complete remission was observed in $59 \%$ of the Ctn-screened group and in $2.7 \%$ of the control group ( $p=0.0001)(177)$.

In another study of patients with thyroid nodules, basal serum Ctn measurements were less than $10 \mathrm{pg} / \mathrm{mL}$ in 5535 (95.2\%) of 5817 patients (179). The remaining 282 patients, 216 with basal Ctn levels between 10 and $20 \mathrm{pg} / \mathrm{mL}$ were followed with yearly Ctn measurements. One of the 216 patients had a thyroidectomy when the basal Ctn level rose to $33 \mathrm{pg} / \mathrm{mL} 2$ years after initial testing and $\mathrm{CCH}$ was present at thyroidectomy. Of the remaining 66 patients who had basal serum Ctn levels above $20 \mathrm{pg} / \mathrm{mL}$, nine had basal Ctn levels above $100 \mathrm{pg} / \mathrm{mL}$, and at thyroidectomy, MTC was confirmed histologically in each of them. The remaining 57 patients had pentagastrin stimulation testing, which was positive (exceeded $100 \mathrm{pg} / \mathrm{mL}$ ) in four of eight patients with basal Ctn levels between 50 and $100 \mathrm{pg} / \mathrm{mL}$ (the thyroidectomy specimen showed MTC in two and $\mathrm{CCH}$ in two), and in 8 of 49 patients with basal Ctn levels between 20 and $50 \mathrm{pg} / \mathrm{mL}$ (the thyroidectomy specimen showed MTC in four and $\mathrm{CCH}$ in four).

The low rate of cure once disease spreads beyond the thyroid gland supports the role of Ctn screening in the early diagnosis of MTC in patients with thyroid nodules. However, given that MTC is present in only $0.3 \%-1.4 \%$ of patients with thyroid nodules, routine serum $\mathrm{Ctn}$ measurement in this population has raised concerns of cost-effectiveness, especially when many of the operated patients would have no MTC based on imperfect specificity if a cutoff was chosen that optimized sensitivity. Additionally, the clinical significance and natural history of MTC diagnosed by Ctn screening is unknown. Although, this practice is the standard of care at selected centers in European countries there has been controversy regarding its acceptance in the United States $(180,181)$. Even though recent studies show that $\mathrm{Ctn}$ measurement of thyroid nodule FNA washings may significantly improve the sensitivity of the test, questions of costeffectiveness are likely to remain $(176,182,183)$.
Concern exists that previous studies of serum Ctn levels in patients with thyroid nodules are potentially biased because the results of subjects prospectively accrued are compared to results of historical controls. There are no prospective randomized trials evaluating the efficacy of serum Ctn screening to standard evaluation of patients with nodular goiters. Also, none of the cited studies address the issues of morbidity and potential complications associated with thyroidectomy in patients with abnormal serum Ctn levels who ultimately prove to have no MTC. Does the earlier diagnosis of MTC in a very small number of patients with nodular goiters justify a total thyroidectomy with its attendant complications in the larger pool of individuals who have no MTC? The recommendations of the Task Force regarding the use of serum Ctn screening in patients with nodular goiters were mixed and primarily based on the member's country of origin.

\section{- RECOMMENDATION 20}

Realizing that opinions of experts vary regarding the usefulness of measuring serum Ctn levels in patients with nodular goiters, the Task Force recommends that physicians decide whether the technique is useful in the management of patients in their clinic. Grade I Recommendation

\section{[I] MANAGEMENT OF PATIENTS WITH A THYROID NODULE AND HISTOLOGICAL DOCUMENTATION OF MTC (FIGURE 1)}

\section{[I-1] Preoperative imaging studies}

Patients who present with a newly detected thyroid nodule and histological confirmation of MTC should have a physical examination and direct DNA analysis of blood cells to detect the presence of a RET germline mutation. If hereditary MTC is evident, PHEOs and HPTH should be excluded. The presence of bilateral MTC does not necessarily ensure that the tumor is hereditary since the frequency of disease in both thyroid lobes ranges from $0 \%$ to $9 \%$ in patients with sporadic MTC who have no RET germline mutations (184-186).

In a study of 300 patients evaluated prior to thyroidectomy for MTC, no distant metastases were detected when the baseline serum Ctn level was less than $500 \mathrm{pg} / \mathrm{mL}$ (187). However, regardless of a patient's serum Ctn level, preoperative systemic imaging is indicated when there is extensive neck disease, or signs or symptoms of distant metastases.

Ultrasound (US) evaluation of the neck is the most important preoperative imaging study in patients with thyroid cancer. If metastatic MTC is expected preoperatively, additional imaging procedures are indicated. Computed tomography (CT) is the most sensitive imaging procedure to detect lung and mediastinal lymph node metastases. Three-phase contrast-enhanced multi-detector liver CT and contrast enhanced magnetic resonance imaging (MRI) are the most sensitive methods to detect liver metastases. Axial MRI and bone scintigraphy are complementary and the most sensitive procedures to detect bone metastases (188). 2- $\left[{ }^{18} \mathrm{~F}\right]$-fluoro-2-deoxy-D-glucose (FDG) positron emission tomography/CT (FDG-PET/CT) and ${ }^{18}$ F-dihydroxyphenylalanine F-DOPA-PET/CT are less sensitive in detecting metastases, compared to other imaging procedures (188). Unfortunately, no single procedure provides optimal wholebody imaging. 


\section{- RECOMMENDATION 21}

Patients presenting with a thyroid nodule and a cytological or histological diagnosis of MTC should have a physical examination, determination of serum levels of Ctn and CEA, and genetic testing for a RET germline mutation. The presence of a PHEO and HPTH should be excluded in patients with hereditary MTC. Grade B Recommendation

\section{- RECOMMENDATION 22}

Ultrasound examination of the neck should be performed in all patients with MTC. Contrast-enhanced CT of the neck and chest, three-phase contrast-enhanced multi-detector liver $\mathrm{CT}$, or contrast-enhanced MRI of the liver, and axial MRI and bone scintigraphy are recommended in patients with extensive neck disease and signs or symptoms of regional or distant metastases, and in all patients with a serum Ctn level greater than $500 \mathrm{pg} / \mathrm{mL}$. Grade C Recommendation

\section{- RECOMMENDATION 23}

Neither FDG-PET/CT nor F-DOPA-PET/CT is recommended to detect the presence of distant metastases. Grade E Recommendation

\section{[1-2] The initial surgical treatment of patients with MTC}

Total thyroidectomy and dissection of cervical lymph node compartments, depending on serum Ctn levels and US findings, is standard treatment for patients with sporadic or hereditary MTC. Patients with a palpable thyroid nodule have a high rate of lymph node metastases that often elude detection preoperatively or even intraoperatively. In one study lymph node metastases were detected in 27 (75\%) of 36 patients with MTC during total thyroidectomy and central and lateral compartment neck dissections (189). Similarly, in another study lymph node metastases were found in greater than $75 \%$ of 73 patients with MTC treated by total thyroidectomy and immediate or delayed central and bilateral functional neck dissection (190). Patients with unilateral intrathyroidal tumors had lymph node metastases in $81 \%$ of central compartment (level VI) dissections, $81 \%$ of ipsilateral lateral compartment (levels II to V) dissections, and $44 \%$ of contralateral lateral compartment (levels II to V) dissections. In patients with bilateral intrathyroidal tumors, nodal metastases were present in $78 \%$ of central compartment dissections, $71 \%$ of lateral compartment dissections ipsilateral to the largest intrathyroid tumor, and $49 \%$ of lateral compartment dissections contralateral to the largest thyroid tumor. Surprisingly, the frequency of lymph node metastases in the central and ipsilateral compartments ranged from $50 \%$ to $75 \%$, whether the primary tumor was less than $1 \mathrm{~cm}$ or greater than $4 \mathrm{~cm}$. The sensitivity of the surgeon's intraoperative assessment of nodal metastases was $64 \%$, and the specificity was $71 \%$ (190).

The pattern of lymph node metastases, as demonstrated for PTC, may also apply to MTC depending on the size and location of the primary tumor. Tumors located in the upper thyroid pole metastasize first to the upper portion of the ipsilateral lateral compartment, whereas tumors in the middle and lower portions of the gland spread first to the central compartment (191). Tumors in the upper pole of the gland, involving the lateral compartment nodes on the ipsilateral side, skip the central compartment in approximately $25 \%$ of cases (192). Apart from the location of the primary intrathyroidal tumor, the overall incidence of lateral compartment lymph node metastases is related to the frequency of central compartment lymph node metastases. In a study of 195 patients with MTC the rate of ipsilateral lateral compartment lymph node metastases was $10.1 \%, 77 \%$, or $98 \%$, when no central compartment lymph node metastases were present, when one to three central compartment lymph node metastases were present, or when four or more central compartment lymph node metastases were present, respectively. The rate of contralateral lateral compartment lymph node metastases was $4.9 \%, 38 \%$, or $77 \%$ when no central compartment lymph node metastases were present, when one to nine central compartment lymph node metastases were present, or when 10 or more central compartment lymph node metastases were present, respectively (193). With ipsilateral lymphatic drainage of tumor cells (i.e., involvement of only the central and lateral cervical lymph node compartments on the side of the primary neoplasm), surgical cure may be attainable in some patients, whereas metastases in the contralateral lateral compartment herald incurable disease (194).

The preoperative basal serum Ctn level is also useful in determining the extent of lymph node metastases. In a study of 300 consecutive patients with MTC treated by total thyroidectomy and compartment-oriented lymph node dissections, there was virtually no risk of lymph node metastases when the preoperative serum Ctn level was less than $20 \mathrm{pg} / \mathrm{mL}$ (normal reference range $<10 \mathrm{pg} / \mathrm{mL}$ ) (187). Basal serum Ctn levels exceeding 20, 50,200 , and $500 \mathrm{pg} / \mathrm{mL}$ were associated, respectively, with metastases to lymph nodes in the ipsilateral central and ipsilateral lateral neck, the contralateral central neck, the contralateral lateral neck, and the upper mediastinum. Bilateral compartmentoriented neck dissection achieved postoperative biochemical cure in at least half of the patients with pretreatment basal Ctn levels of $1000 \mathrm{pg} / \mathrm{mL}$ or less, but not in patients with levels greater than $10,000 \mathrm{pg} / \mathrm{mL}$ (187). Thus, although imperfect, the determination of basal serum Ctn levels provides useful information for the operative management of patients with MTC. It should be noted that patients whose basal serum calcitonin level is normal $(<10 \mathrm{pg} / \mathrm{mL})$ following attempted complete lymph node dissection are said to be "biochemically cured" and have a $97.7 \%$ survival at 10 years (37). However, $3 \%$ of patients with a normal baseline serum Ctn level following thyroidectomy will have a biochemical recurrence within 7.5 years (195).

Important information can be gained also from preoperative serum CEA levels. In a study of 150 patients, 54 of whom had preoperative serum CEA levels greater than $4.7 \mathrm{ng} / \mathrm{mL}$, there was a respective significant association between successive increases of serum CEA (4.7-10, 10.1-30, 30.1$100 \mathrm{ng} / \mathrm{mL}$, and $>100 \mathrm{ng} / \mathrm{mL}$ ) and the presence of 10 or more positive cervical lymph nodes; $0 \%, 9 \%, 53 \%$, and $69 \%$ $[p<0.001])$, involvement of cervical lymph node compartments (central: 33\%, 36\%, 73\%, and 93\% [ $p=0.002]$; lateral [ipsilateral]: $20 \%, 27 \%, 67 \%$, and $88 \%$ [ $p=0.001$ ]; lateral [contralateral]: $22 \%, 10 \%, 36 \%$, and $73 \%[p=0.008])$, the presence of distant metastasis $(0 \%, 27 \%, 13 \%$, and $75 \%[p<0.001])$, and postoperative normalization of serum Ctn levels (biochemical cure) $(58 \%, 33 \%, 7 \%$, and $0 \%[p=0.001])(196)$.

Considering the complications associated with repeat neck operations, the surgeon should dissect the necessary lymph node compartments during the initial thyroidectomy, based on the frequency and pattern of lymph node metastases relative to the location and size of the primary MTC, US 
findings, and serum levels of Ctn and CEA. Clearance of lymph nodes in the lateral neck is not without complications, primarily lymphatic leakage, occurring in $0.5 \%-8 \%$ of patients, and damage to a spinal accessory nerve with resulting shoulder dysfunction, occurring in 25\%-50\% of patients (197-199).

There are two schools of thought regarding the use of preoperative studies in the management of lymph node compartments at thyroidectomy. Some endocrinologists and surgeons consider preoperative US of primary importance in detecting lymph node metastases and do not advocate compartment dissection if US of the neck is negative. Others argue that elective dissection of US-normal ipsilateral central and ipsilateral lateral neck compartments is indicated in patients with basal serum Ctn levels above $20 \mathrm{pg} / \mathrm{mL}$. Also, elective dissection of an US-normal contralateral lateral neck compartment is indicated when the basal serum Ctn level is greater than $200 \mathrm{pg} / \mathrm{mL}$ (187).

\section{- RECOMMENDATION 24}

Patients with MTC and no evidence of neck lymph node metastases by US examination and no evidence of distant metastases should have a total thyroidectomy and dissection of the lymph nodes in the central compartment (level VI). Grade B Recommendation

\section{- RECOMMENDATION 25}

In patients with MTC and no evidence of neck metastases on US, and no distant metastases, dissection of lymph nodes in the lateral compartments (levels II-V) may be considered based on serum Ctn levels. The Task Force did not achieve consensus on this recommendation. Grade I Recommendation

\section{- RECOMMENDATION 26}

Patients with MTC confined to the neck and cervical lymph nodes should have a total thyroidectomy, dissection of the central lymph node compartment (level VI), and dissection of the involved lateral neck compartments (levels II-V). When preoperative imaging is positive in the ipsilateral lateral neck compartment but negative in the contralateral neck compartment, contralateral neck dissection should be considered if the basal serum calcitonin level is greater than 200 pg/mL. Grade C Recommendation

\section{[J] RESULTS OF THYROIDECTOMY IN PATIENTS WITH MTC}

Unfortunately, most patients with MTC and regional lymph node metastases have systemic disease and are not cured by total thyroidectomy and bilateral neck dissection $(200,201)$. In a study of 534 patients with MTC treated by total thyroidectomy and neck dissection, both decreased lymph node yield and an increased ratio of metastatic lymph nodes to the total number of lymph nodes resected appeared to be associated with poor survival (202). In another study metastases to 10 or more lymph nodes or involvement of more than two lymph node compartments precluded normalization of serum Ctn (203). Quantitative assessment of lymph node metastases, 1-10 (N1), 11-20 (N2), and more than $20(\mathrm{~N} 3)$, is an important prognostic classifier that should be incorporated into the AJCC staging systems, which currently includes N1a and $\mathrm{NIb}$ categories referring only to qualitative involvement of lymph node compartments (Table 5) (204).
Table 5. American Joint Committee on Cancer TNM Classification (Thyroid CANCER)

Description

\begin{tabular}{cc}
\hline Primary tumor $(\mathrm{T})^{\mathrm{a}}$ \\
TX & Primary tumor cannot be assessed \\
T0 & No evidence of primary tumor \\
T1 & Tumor $2 \mathrm{~cm}$ or less in greatest \\
& dimension, limited to the thyroid \\
T1a & Tumor $1 \mathrm{~cm}$ or less, limited to the thyroid \\
T1b & Tumor more than $1 \mathrm{~cm}$, but not more than \\
& $2 \mathrm{~cm}$, in greatest dimension, limited to \\
the thyroid & Tumor more than $2 \mathrm{~cm}$, but not more \\
T2 & than $4 \mathrm{~cm}$, in greatest dimension, \\
& limited to the thyroid \\
T3 & Tumor more than $4 \mathrm{~cm}$ in greatest dimension \\
& limited to the thyroid, or any tumor with \\
& minimal extrathyroid extension \\
& (e.g., extension to sternothyroid muscle \\
& or perithyroid soft tissues) \\
T4a & Moderately advanced disease; tumor of any \\
& size extending beyond the thyroid capsule \\
& to invade subcutaneous soft tissues, \\
& larynx, trachea, esophagus, or recurrent \\
laryngeal nerve & \\
T4b & Very advanced disease; tumor invades \\
& prevertebral fascia or encases carotid \\
& artery or mediastinal vessels
\end{tabular}

Regional lymph nodes $(\mathrm{N})^{\mathrm{b}}$

NX Regional lymph nodes cannot be assessed

NO No regional lymph node metastasis

N1 Regional lymph node metastasis

N1a Metastasis to level VI (pretracheal, paratracheal, and prelaryngeal/Delphian lymph nodes)

N1b Metastasis to unilateral, bilateral, or contralateral cervical (levels I, II, III, IV, or V) or retropharyngeal or superior mediastinal lymph nodes (level VII)

Distant metastases (M)

M0 No distant metastasis

M1 Distant metastasis

Anatomic stage/prognostic groups

Stage I T1, N0, M0

Stage II T2, N0, M0

T3, N0, M0

Stage III T1, N1a, M0

T2, N1a, M0

T3, N1a, M0

Stage IVA T4a, NO, M0

T4a, N1a,M0

T1, N1b, M0

T2, N1b, M0

T3, N1b, M0

T4a, N1b, M0

Stage IVB T4b, any N, M0

Stage IVC Any T, any N, M1

Modified from the 7th Edition of the American Joint Committee on Cancer Staging Manual, with permission of Springer-Verlag New York, Inc., 175 Fifth Avenue, New York, NY 10010, USA.

${ }^{\mathrm{a}}$ All categories may be subdivided: (s) solitary tumor and (m) multifocal tumor (the largest determines the classification).

${ }^{\mathrm{b}}$ Regional lymph nodes are the central compartment and the lateral cervical and upper mediastinal lymph node compartments. 
Despite the inability to cure all patients with regional lymph node metastases, the resection of local metastases may decrease the risk of local recurrence. Also, the clearance of central and lateral compartments may prevent future complications such as invasion of recurrent laryngeal nerves, the aerodigestive tract, or lateral neck nerves with respective loss of speech, altered swallowing, and arm or shoulder dysfunction $(205,206)$. For these reasons, most surgeons recommend a compartmentoriented lymph node dissection at the primary thyroidectomy if metastatic lymph nodes are evident $(202,207)$.

\section{[K] MANAGEMENT OF PATIENTS WITH LOCALLY ADVANCED OR METASTATIC MTC}

In most patients with MTC the goal is to perform a total thyroidectomy, with or without compartment-oriented lymph node dissections. However, in the presence of advanced disease the goals of surgery are more palliative with attention to minimizing complications. In the presence of MTC that invades the trachea, thyroid cartilage, or esophagus, the extent of extirpative surgery (palliative debulking, laryngectomy, esophagectomy, or laryngopharyngectomy) is determined by an assessment of the ability to maintain speech and swallowing and the patient's life expectancy based on the extent of disease and other medical comorbidities. These decisions are best made on an individualized basis by an experienced multidisciplinary medical team, including the patient.

\section{- RECOMMENDATION 27}

In the presence of extensive regional or metastatic disease less aggressive surgery in the central and lateral neck may be appropriate to preserve speech, swallowing, parathyroid function, and shoulder mobility. External beam radiotherapy (EBRT), systemic medical therapy, and other nonsurgical therapies should be considered to achieve local tumor control. Grade C Recommendation

\section{[L] MANAGEMENT OF PATIENTS FOLLOWING AN INCOMPLETE THYROIDECTOMY AND LYMPH NODE DISSECTION}

Occasionally, the diagnosis of MTC is made following a unilateral hemithyroidectomy. The opposite thyroid lobe should be removed in patients with hereditary MTC because the likelihood that MTC is already present or will develop in the future approaches $100 \%$. In patients with sporadic MTC the incidence of bilateral MTC ranges from $0 \%$ to $9 \%$, and there are few studies evaluating patient management in this setting $(187,188)$. In a prospective study of 15 patients treated by hemithyroidectomy for sporadic MTC, 12 (80\%) achieved a biochemical cure as defined by a postoperative serum Ctn level within the normal range following calcium and pentagastrin stimulation. Metastases to regional nodes and large tumor size were adversely related to biochemical cure (186).

Completion thyroidectomy following hemithyroidectomy is not indicated unless the patient has a RET germline mutation, a significant elevation of basal or stimulated serum $\mathrm{Ctn}$ levels postoperatively, or imaging studies showing residual MTC. For simplicity, serum Ctn levels above the upper normal value of the reference range may be a reasonable level at which completion thyroidectomy should be recommended. However, there are no data on which to base this recommendation, and the decision to follow a patient with sporadic MTC after hemithyroidectomy without further surgery should be made on an individual basis, weighing the potential risks and benefits of additional surgery compared to watchful waiting. During follow-up, a progressive increase in the basal serum Ctn level above $150 \mathrm{pg} / \mathrm{mL}$ should prompt additional imaging.

Patients with residual lymph node metastases after thyroidectomy are likely to benefit from re-operation. In a study of 334 patients a repeat operation was performed for persistent MTC, as evidenced by elevated serum Ctn levels (1797 pg/mL [885-2736 pg/mL]), (208). Systematic central and lateral lymph node dissection attained biochemical cure in $59(44 \%)$ of 133 patients who had no metastases in lymph nodes removed at prior surgery. Conversely, biochemical cure was attained in only $12(18 \%)$ of 65 patients in whom one to five metastatic lymph nodes had been removed previously (208). If more than five metastatic lymph nodes were resected at prior surgery the biochemical cure rate fell to $5 \%$ ( 2 of 43 patients). When preoperative basal serum Ctn levels exceeded $1000 \mathrm{pg} / \mathrm{mL}$ (reference range $<10 \mathrm{pg} / \mathrm{mL}$ ) biochemical cure was exceptional (1 of 76 patients). It is noteworthy that $18 \%$ of the 334 patients were referred with unilateral permanent recurrent laryngeal nerve palsy, presenting a challenge in the dissection of the contralateral neck (208). Recently, surgeons have used continuous intraoperative nerve monitoring during thyroidectomy for primary or recurrent MTC. The technique signals impending nerve injury, most often due to traction on the nerve, and allows timely corrective action (209). However, there have been no multicenter, prospective, randomized trials comparing this technique to thyroidectomy alone. These data suggest that systematic lymph node dissection in patients who had an inadequate lymphadenectomy at initial thyroidectomy is worthwhile, as long as the preoperative serum Ctn level is less than $1000 \mathrm{pg} / \mathrm{mL}$ and five or fewer metastatic lymph nodes were resected initially. Beyond these thresholds, the focus of surgical treatment shifts to the maintenance of local control in the neck.

\section{- RECOMMENDATION 28}

Following unilateral thyroidectomy for presumed sporadic MTC completion thyroidectomy is recommended in patients with a RET germline mutation, an elevated postoperative serum Ctn level, or imaging studies indicating residual MTC. The presence of an enlarged lymph node in association with a normal serum $\mathrm{Ctn}$ level is not an indication for repeat surgery. Grade B Recommendation

\section{- RECOMMENDATION 29}

In patients having an inadequate lymph node dissection at the initial thyroidectomy a repeat operation, including compartment oriented lymph node dissection, should be considered if the preoperative basal serum CTN level is less than $1000 \mathrm{pg} /$ $\mathrm{mL}$ and five or fewer metastatic lymph nodes were removed at the initial surgery. Grade $\mathrm{C}$ Recommendation

\section{[M] MANAGEMENT OF NORMAL PARATHYROID GLANDS RESECTED OR DEVASCULARIZED DURING SURGERY}

The surgeon should be experienced in identifying parathyroid glands and have expertise with parathyroid autotransplantation. Normal parathyroid glands can be accidentally removed or devascularized during thyroidectomy or central neck lymph 
node dissection. Whenever possible, viable parathyroid tissue should be left in situ on a vascular pedicle. If the blood supply to a parathyroid gland cannot be preserved during thyroidectomy, or if the status of the blood supply is questionable or clearly inadequate, parathyroid tissue should be grafted to the sternocleidomastoid muscle in patients with sporadic MTC and MEN2B and in patients with MEN2A who have a RET mutation rarely associated with HPTH. Otherwise, the parathyroid tissue should be grafted to a heterotopic muscle bed, such as the bracheoradialis, where portions of the graft can be removed if graftdependent hyperparathyroidism develops (210).

\section{- RECOMMENDATION 30}

During a total thyroidectomy for MTC, normal parathyroid glands should be preserved in situ on a vascular pedicle. If all normal parathyroid glands are resected or if none appear viable at the termination of the procedure, slivers of a parathyroid gland should be transplanted into the sternocleidomastoid muscle in patients with sporadic MTC, MEN2B, or MEN2A and a RET mutation rarely associated with HPTH. In patients with MEN2A and a RET mutation associated with a high incidence of HPTH, the parathyroid tissue should be transplanted in a heterotopic muscle bed. Grade B Recommendation

\section{[N] HORMONE REPLACEMENT FOLLOWING THYROIDECTOMY}

Following thyroidectomy there is no reason to suppress thyrotropin (TSH) since the MTC is not a follicular neoplasm; however, replacement L-thyroxine is needed to maintain serum TSH levels in the euthyroid range. Also, patients need to be monitored for hypocalcemia, which is almost always transient; however, treatment with oral cal- cium and calcitriol is indicated if patients become symptomatic or have persistently prolonged hypocalcemia. Withdrawal of replacement therapy is guided by serial measurements of serum calcium.

\section{- RECOMMENDATION 31}

Serum TSH should be measured within 4-6 weeks postoperatively. Replacement therapy with levothyroxine should be administered with the goal of maintaining serum TSH levels in the euthyroid range. Grade B Recommendation

\section{- RECOMMENDATION 32}

Serum calcium levels should be monitored postoperatively. Oral calcium and vitamin D should be administered to patients who develop symptomatic hypocalcemia. Chronic replacement therapy is indicated in patients who cannot be weaned from medication. Grade B Recommendation

\section{[O] PROPHYLACTIC THYROIDECTOMY IN CHILDREN WITH HEREDITARY MTC (FIGURE 2)}

Prophylactic surgery is most applicable for patients with hereditary cancer syndromes and ideally should meet the following criteria: 1) the genetic mutation causing the malignancy is characterized by complete or near complete penetrance; 2) there is a highly reliable test for detecting the mutation; 3) the organ at risk is expendable, or if not expendable, there is therapy to replace the function of the organ; 4) the precancerous organ can be removed with minimal morbidity and virtually no mortality; and 5) there is a reliable test to determine if the operation has been curative. Many hereditary cancer syndromes meet some, but not all of these criteria, whereas MEN2A and MEN2B meet each of them. In patients with hereditary MTC, there is a classic progression

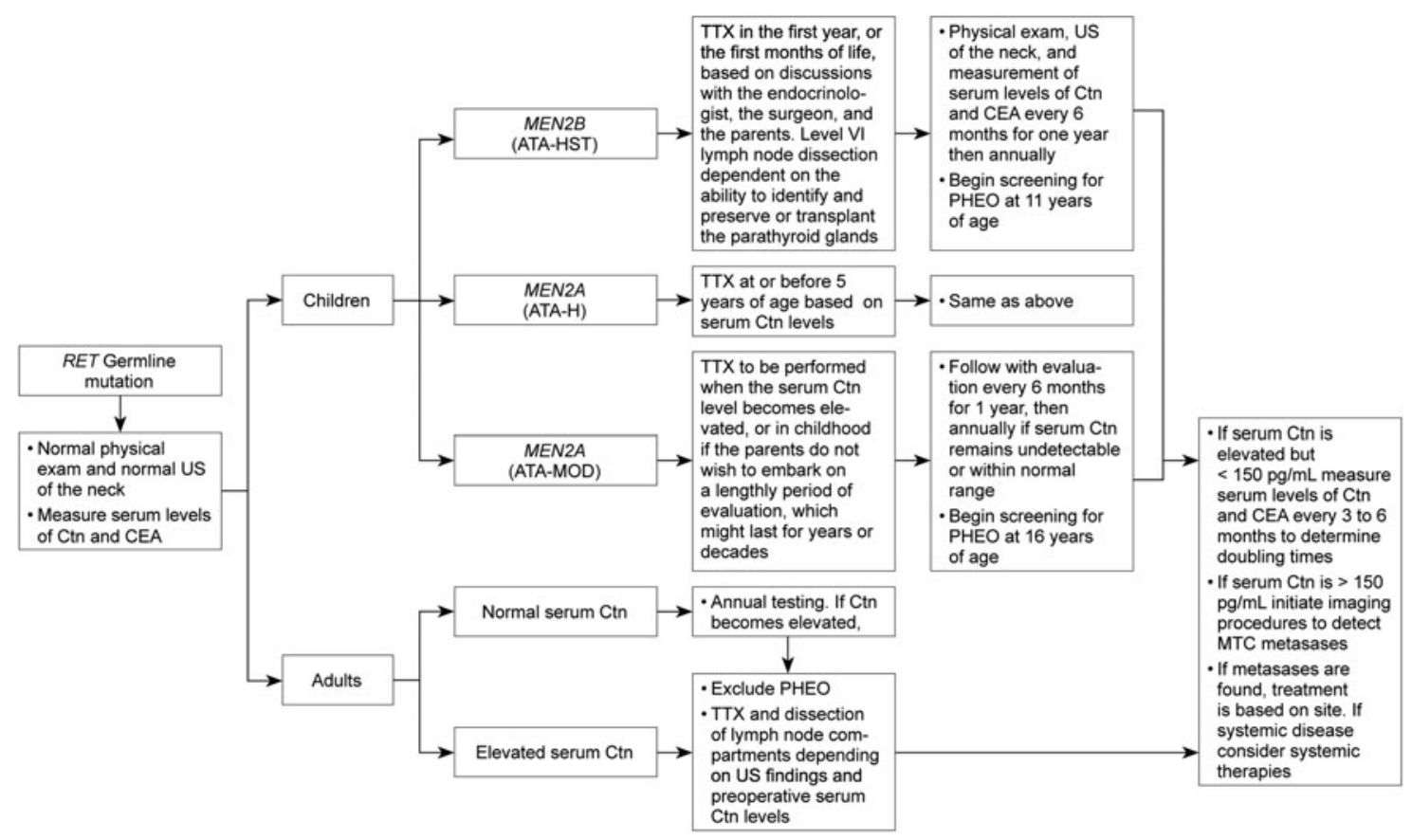

FIG. 2. Management of patients with a RET germline mutation detected on genetic screening. ATA, American Thyroid Association risk categories for aggressive medullary thyroid carcinoma (MTC) (HST, highest risk, H, high risk, MOD, moderate risk); Ctn, calcitonin; CEA, carcinoembryonic antigen; HPTH, hyperparathyroidism; PHEO, pheochromocytoma; RET, REarranged during Transfection; TTX, total thyroidectomy; US, ultrasound. 
from $\mathrm{CCH}$, to MTC, to loco-regional lymph nodes, and ultimately to distant sites, a process that occurs from months to years and largely depends on the RET mutation (64,211). In this setting it is possible to remove the thyroid before it becomes cancerous, or if cancer has developed it is confined to the gland.

The term "prophylactic thyroidectomy" has been used to describe early removal of the thyroid gland in children who have inherited a mutated RET allele; however, in most cases $\mathrm{CCH}$ or frank MTC is already present at the time of thyroidectomy. In the present context we use the term "prophylactic" to define removal of the thyroid before MTC develops or while it is clinically unapparent and confined to the gland. Timely thyroidectomy for MTC favorably alters the associated morbidity and mortality, thus, the question is not whether prophylactic thyroidectomy should be performed in patients with hereditary MTC, but at what age.

Prior to the introduction of direct DNA analysis to detect a mutated RET allele the diagnosis of hereditary MTC was based on determination of the serum Ctn level. Although a C-cell disorder could be detected at an early age with biochemical testing, there were no generally accepted criteria specifying the serum Ctn level at which thyroidectomy should be performed. Expectedly, some children were subjected to thyroidectomy based on the serum Ctn level only to learn years later on direct DNA analysis that they had not inherited a RET mutation (64,212,213). With the discovery that mutations in the RET oncogene cause the MEN2 syndromes and the recognition that there is a correlation between genotype and phenotype, the focus on early diagnosis shifted to direct DNA analysis. It soon became apparent, however, that there was great heterogeneity in the age of onset and aggressiveness of MTC, not only among different families with the same RET mutation (excepting mutations in RET codon 634 and codon 918), but within individual families having the same RET mutation.

Given our evolving understanding of hereditary MTC, the decision regarding the age of prophylactic thyroidectomy is no longer based on direct DNA analysis alone, rather it is driven by additional clinical data, the most important being the basal or stimulated serum Ctn level (214). Given the lack of generally accepted guidelines relating specific basal or stimulated serum Ctn levels to the development of a C-cell disorder it is important that clinicians become familiar with the reference range of the $\mathrm{Ctn}$ assay in their medical center and establish criteria for recommending thyroidectomy in children known to have inherited a mutated RET allele. The management plan for a child with hereditary MTC should be communicated to and understood by the parents.

Compared to adults, children, and especially infants, have higher complication rates associated with thyroidectomy, the most significant being hypoparathyroidism. The parathyroid glands are often hard to identify in very young children because they are small, translucent, and difficult to distinguish from surrounding tissues. Even though complications are significantly minimized when experienced surgeons perform the thyroidectomy $(215,216)$, one might consider delaying the procedure in children less than 2 years of age. Also, some pediatricians have concern that potential detrimental effects of insufficient thyroid hormone replacement, perhaps due to noncompliance, have attendant consequences in young children, such as impaired brain development and retarded growth (217).
Nevertheless, there are significant risks in delaying surgery in family members who have inherited a mutated RET allele, and regardless of the patient's age, one must balance the risks of thyroidectomy against the possibility that the MTC will not be cured if thyroidectomy is delayed. This conundrum is exemplified by a family with MEN2A and the RET codon L790F mutation. Of 22 family members 15 had a $R E T$ codon L790F mutation and 8 of the 15 had elevated basal or stimulated serum Ctn levels. Five patients (45-76 years of age) with elevated serum Ctn levels and clinical stage pT1N0M0 disease were cured biochemically following thyroidectomy and lymph node dissection. The three remaining patients with elevated serum Ctn levels refused surgery. Seven patients with serum Ctn levels less than $30 \mathrm{pg} / \mathrm{mL}$ did not have surgery and were followed expectantly. The follow-up $(<5$ years) in the seven patients was complicated, because three of them considered it impractical to continue periodic evaluations, or for other reasons chose not to do so (218). This is a significant risk factor for clinicians and their patients who choose to base the timing of thyroidectomy on long-term periodic physical examinations and measurement of serum Ctn levels. In time, patients may tire of repeat evaluations and discontinue periodic clinic visits, only to be seen again, perhaps years later, with clinically evident disease.

\section{[0-1] Prophylactic thyroidectomy for children with MEN2A (Figure 2)}

Children with MEN2A and RET codon 634 mutations (ATA-H category) often develop MTC during the first years of life; therefore, annual physical examination, cervical US, and measurement of serum Ctn levels should begin at 3 years of age (219). Children in the ATA-MOD category, compared to children in the ATA-H category, typically develop a less aggressive MTC at a later age. The behavior of the tumor can be variable, however, as evidenced by a child with a $R E T$ codon 804 mutation who died of metastatic MTC at 12 years of age (219-221). Although much can be learned from studying several families with the same RET mutation, there may be substantial variability among families not only in the age of onset and the aggressiveness of the MTC, but in the clinical expression of the disease spectrum. For example, in studies of various families with the RET codon C609S mutation the development of $\mathrm{CCH}$ or MTC ranged from 9 to 48 years of age, and with the exception of one family, the mean age of MTC onset was 20 years. Also, in some families with the RET codon C609S mutation no one developed a PHEO, while in other families not only were there PHEOs, but in one kindred PHEO was the dominant endocrinopathy, there being no clinically evident MTC (222-225). Considering the clinical variability of disease expression in family members in the ATA-MOD category, annual physical examination, cervical US, and measurement of serum Ctn levels, should begin at 5 years of age.

Several international groups have reported their experience with prophylactic thyroidectomy in children with MEN2A (217,226-233). In one series of 46 children, five $(9.2 \%)$ children, all over 13 years of age (with RET mutations in codons 620, 630, 634, 634, and 634), had persistent or recurrent MTC following prophylactic thyroidectomy (217). In another series 50 children were evaluated 5 or more years after total thyroidectomy and central zone neck dissection by 
measurement of serum Ctn levels following stimulation with combined calcium and pentagastrin. There was no persistent or recurrent MTC in $44(88 \%)$ children (one with lymph node metastases). The remaining six children, ages $8,10,11,14$, 16, and 19 years (with RET mutations in codons 618, 618, $620,620,634$, and 634) developed persistent or recurrent MTC postoperatively, as indicated by a detectable basal or stimulated serum Ctn level. Two of the six children had lymph node metastases (227).

The MTC that occurs in young children with MEN2A is often small and in one study there were no lymph node metastases or evidence of residual MTC when the basal Ctn was less than $30 \mathrm{pg} / \mathrm{mL}$ preoperatively or when the primary tumor was smaller than $10 \mathrm{~mm}$ (234). In other studies there were no lymph node metastases at thyroidectomy if the basal preoperative serum Ctn level was below $40 \mathrm{pg} / \mathrm{mL}$ (234-236). In an additional two studies lymph node metastases were uncommon before 11 years of age $(227,237)$. Thus, when the preoperative basal serum Ctn is less than $40 \mathrm{pg} / \mathrm{mL}$, a total thyroidectomy without central (level VI) neck dissection is adequate therapy. Such a strategy should reduce the risk of damage to the parathyroid glands and the laryngeal nerves.

Ultimately, almost all patients with MEN2A require thyroidectomy. Children in the ATA-H category should have a thyroidectomy at or before 5 years of age, with the timing and extent of surgery guided by serum Ctn levels. Children in the ATA-MOD category should have a thyroidectomy in childhood or young adulthood, the timing depending primarily on serum Ctn levels. It is important that the pediatrician and surgeon caring for the child collaborate with the child's parents to plan the time for thyroidectomy. Some parents may wish to proceed with thyroidectomy during childhood rather than opt for long-term evaluation, which might last for decades.

\section{[0-2] Prophylactic thyroidectomy for children with MEN2B (Figure 2)}

In patients with MEN2B and a RET codon M918T mutation (ATA-HST category), the MTC is usually highly aggressive and if possible thyroidectomy should be performed early in life. Two groups of patients with MEN2B should be considered separately: the $25 \%$ or fewer who have known hereditary MEN2B and the $75 \%$ or more who have de novo RET mutations and phenotypically normal parents.

In children with hereditary MEN2B, macroscopic MTC and nodal metastases may occur during the first year of life (238). For these reasons, genetic testing should be done soon after birth in at-risk infants. Children who have inherited MEN2B should have a thyroidectomy in the first year of life, perhaps even in the first months of life, the exact timing determined by the surgeon and pediatrician in consultation with the child's parents $(238,239)$. There are complicating factors in infants. Serum Ctn levels are extremely high in the first months of life and of limited value in timing thyroidectomy. More importantly, the parathyroid glands can be very difficult to identify intraoperatively, creating an inordinate risk of hypoparathyroidism. In the absence of suspicious lymph nodes, the decision to perform a central neck dissection should be based on whether the parathyroid glands are identified and can either be preserved in situ or autotransplanted. The surgeon should consider foregoing a central neck dissection if he cannot identify the parathyroid glands.
In children with de novo RET codon M918T mutations the diagnosis of MEN2B is usually made upon detection of a thyroid nodule $(240,241)$. In one study of 21 patients with $d e$ novo MEN2B and a RET codon M918T mutation the mean age at diagnosis was 14.2 years (range 1-31 years). In 18 of the 21 patients the mean preoperative basal serum Ctn level was $26,080 \mathrm{pg} / \mathrm{mL}$ (range $35-105,000 \mathrm{pg} / \mathrm{mL}$ ), and in $4(18 \%)$ of the 21 patients serum Ctn levels were within the normal range postoperatively (242). Despite the aggressiveness of MTC in MEN2B, some children are cured by thyroidectomy $(243,244)$. It is critically important that physicians be aware of the characteristic phenotype associated with MEN2B, since it is almost always evident prior to the detection of a thyroid nodule or a PHEO. In a study of 44 children with MEN2B, three patients with hereditary disease had a thyroidectomy during the first year of life and were cured (243). Of the 41 patients with $d e$ novo RET mutations, 12 were diagnosed upon recognition of nonendocrine manifestations of MEN2B: ganglioneuromatosis $(n=6)$, oral symptoms $(n=5)$, ocular manifestations $(n=4)$, and skeletal abnormalities $(n=1)$. The remaining 29 patients were diagnosed because of symptomatic MTC $(n=28)$ or PHEO $(n=1)$. The patients detected because of nonendocrine manifestations, compared to those with symptomatic MTC or PHEO, were significantly younger (5.3 vs. 17.6 years of age; $p<0.001)$, and also had lower preoperative serum Ctn levels (mean 115 vs. $25,519 \mathrm{pg} / \mathrm{mL}$ ), tumors smaller than $10 \mathrm{~mm}(67 \%$ vs. $0 \%)$, a lower incidence of extrathyroidal extension ( $0 \%$ vs. $81 \%$ ), fewer lymph node metastases $(42 \%$ vs. $100 \%$ ), and a lower incidence of distant metastases ( $8 \%$ vs. $79 \%$ ). Most importantly, they were more often cured biochemically following thyroidectomy (58\% vs. 0\%). All nine children having thyroidectomy prior to age 4 years were cured biochemically, compared to only 1 of 35 children having thyroidectomy after age 5 years (244).

Theoretically, very young children with MEN2B could be monitored and thyroidectomy delayed until they are older and the operation is less risky. There have been no studies evaluating the efficacy of such an approach. While central neck dissection may be foregone in very young children with MEN2B, such is not the case in older children with a palpable MTC and elevated serum Ctn levels, since they are at high risk for lymph node metastases and the benefits of central neck dissection outweigh the potential risks associated with the procedure.

In patients with MEN2B who have RET A883F codon mutations, or double mutations involving RET codon V804M and either RET codon Y806C, S904C, E805K, or Q781R, the MTC appears to be less aggressive compared to the MTC in patients with MEN2B and the RET codon M918T mutation. It is difficult to establish management guidelines in these two groups, however, since their mutations are rare and only small numbers of patients have been evaluated.

\section{- RECOMMENDATION 33}

Experienced physicians and surgeons in tertiary care centers should be responsible for the management of children with MEN2A or MEN2B. Grade B Recommendation

\section{- RECOMMENDATION 34}

Children in the ATA-HST category with a RET codon M918T mutation should have a thyroidectomy in the first year of life, perhaps even in the first months of life. In the absence of suspicious lymph nodes the performance of a 
central neck dissection should be based on whether the parathyroid glands can be identified and left in situ or autotransplanted. The surgeon and pediatrician caring for the patient, in consultation with the child's parents, should decide the timing of thyroidectomy. Grade $\mathrm{C}$ Recommendation

\section{- RECOMMENDATION 35}

Children in the ATA-H category should have a thyroidectomy performed at age 5 years, or earlier based on the detection of elevated serum Ctn levels. A central neck dissection should be performed in children with serum Ctn levels above $40 \mathrm{pg} / \mathrm{mL}$, or with evidence on imaging or direct observation of lymph node metastases. The surgeon and pediatrician caring for the patient, in consultation with the child's parents, should decide the timing of thyroidectomy. Grade B Recommendation

\section{- RECOMMENDATION 36}

Children in the ATA-MOD category should have a physical examination, US of the neck, and measurement of serum Ctn levels beginning around 5 years of age. The timing of thyroidectomy should be based on the detection of an elevated serum Ctn level; however, 6-month or annual evaluations may extend to several years or decades. Parents who are concerned about a long-term evaluation program may opt to have their child's thyroid gland removed around 5 years of age. The surgeon and pediatrician caring for the patient, in consultation with the child's parents, should decide the timing of thyroidectomy. Grade B Recommendation

\section{[P] MANAGEMENT OF PHEO IN PATIENTS WITH MEN2A AND MEN2B}

PHEOs usually present in the third or fourth decade of life in patients with MEN2A and the diagnosis is usually made concurrently with or following the diagnosis of MTC. An undiagnosed PHEO in a patient undergoing a thyroidectomy may result in substantial morbidity and even death. Thus, in patients with hereditary MTC, it is critical to exclude the presence of a PHEO prior to thyroidectomy by measuring free plasma or fractionated urinary metanephrines, or both. Either CT or MRI can confirm the location of a PHEO. If both MTC and a PHEO are present in a patient, the PHEO should almost always be removed first. In patients who present with MTC, and it is unknown whether it is sporadic or familial, a PHEO should be excluded prior to thyroidectomy if determination of the RET status takes an inordinate amount of time. A PHEO should also be excluded in patients with MEN2 who wish to become pregnant. A PHEO diagnosed during pregnancy should be resected prior to the third trimester if possible.

Following bilateral adrenalectomy there is a significant risk of adrenal insufficiency, thus unilateral adrenalectomy is indicated in patients with a solitary PHEO, even though the majority of them will develop a contralateral PHEO within 10 years $(63,245)$. Bilateral PHEOs should be resected with corticosteroid coverage intraoperatively and postoperatively. Postoperative surveillance is critical in patients with no adrenal glands and they must carry information on themselves regarding the necessity of glucocorticoid treatment should they become acutely ill or injured.

Laparoscopic adrenalectomy or retroperitoneoscopic adrenalectomy is the procedure of choice for the treatment of unilateral or bilateral PHEOs (246). There appears to be no outcome difference between laparoscopic and retroperitoneoscopic approaches in synchronous bilateral adrenal surgery $(247,248)$. Realizing the risk of Addison crises in patients without adrenocortical tissue, some surgeons have focused on subtotal adrenalectomy (249). Subtotal adrenalectomy that preserves $10 \%-15 \%$ of residual adrenal cortical tissue appears to offer adrenal stress capacity sufficient to obviate the need for corticosteroid supplementation in more than $80 \%$ of patients (250). The risk of recurrent PHEO, however, is $20 \%$ within 20 years after subtotal adrenalectomy (251).

PHEOs may develop as early as 8 and 12 years of age, respectively, in children in the ATA-H and ATA-HST categories, and as early as 19 years of age in children in the ATAMOD category (252-255). In three studies of patients with MEN2, the mean ages at presentation of PHEOs were 25 years (range, 18-40 years), 25.5 years (range, 19-31 years), and 32.4 years (range, 15-41 years), respectively (256-258). The recommended time to begin screening for a PHEO is based on data from the literature including an analysis of 474 patients with hereditary MTC, 84 (17.8\%) of whom developed PHEOs, and $20(4.2 \%)$ of whom developed HPTH (257). Screening for PHEO in children in the ATA-HST, ATA-H, and ATA-MOD categories should begin at 11, 11, and 16 years of age, respectively.

\section{- RECOMMENDATION 37}

Screening for PHEO should begin by age 11 years for children in the ATA-H and ATA-HST categories and by age 16 years in children in the ATA-MOD category. Screening consists of measuring free plasma metanephrines and normetanephrines or 24-hour urinary metanephrines and normetanephrines. Adrenal imaging with CT or MRI is indicated in patients with positive biochemical results. Grade C Recommendation

\section{- RECOMMENDATION 38}

Patients with MEN2A or MEN2B and a histological diagnosis of MTC regardless of age and presenting symptoms must have a PHEO excluded prior to any interventional procedure. The presence of a PHEO must be excluded in women with MEN2A or MEN2B who are planning a pregnancy or are pregnant. If a PHEO is detected it should be treated preferably prior to the third trimester. Grade C Recommendation

\section{- RECOMMENDATION 39}

If they coexist, a PHEO should be removed prior to surgery for either MTC or HPTH. Grade B Recommendation

\section{- RECOMMENDATION $\mathbf{4 0}$}

After appropriate preoperative preparation a PHEO should be resected by laparoscopic or retroperitoneoscopic adrenalectomy. Subtotal adrenalectomy to preserve adrenal cortical function should be considered as an alternative procedure. Grade B Recommendation

\section{- RECOMMENDATION 41}

Patients with no adrenal glands require glucocorticoid and mineralocorticoid replacement therapy and should be carefully monitored to ensure that their steroid levels are adequate. Patients should be educated regarding the risk of 
adrenal crisis and wear a bracelet or a necklace indicating that they have no adrenal glands and are on corticosteroid replacement therapy. Glucocorticoid supplementation will be required if they become severely ill or are injured. Grade B Recommendation

\section{[Q] MANAGEMENT OF HPTH IN PATIENTS WITH MEN2A}

Surveillance for HPTH should include albumin-corrected calcium or ionized serum calcium measurements (with or without serum intact-PTH levels) beginning at age 11 years in patients in the ATA-H category and at age 16 years in patients in the ATA-MOD category. In a study of 188 patients with MEN2A (ATA-H category), 36 had HPTH (259). The mean age at diagnosis of HPTH was 33.7 years (range 12-70 years); however, HPTH has been reported in children in the ATA-H category as early as 2, 6, 7, and 10 years of age (259-262). The HPTH primarily occurs in patients with exon $11 R E T$ codon mutations, most often in those with RET codon 634 mutations, and less frequently in patients with exon 10 RET codon mutations. As more families with MEN2A have been studied, it has become apparent that their HPTH, in contrast to that occurring in families with MEN1, is mild and often asymptomatic $(263,264)$. Enlarged parathyroid glands are occasionally found at the time of thyroidectomy for MTC in patients who are normocalcemic preoperatively $(261,262)$.

Treatment options for patients with HPTH and no prior neck surgery include: 1) subtotal parathyroidectomy leaving one gland or a piece of one gland in situ; 2) total parathyroidectomy with autotransplantation of small slivers of parathyroid tissue to a heterotopic site; or 3) resection of only enlarged glands, with intraoperative parathyroid hormone (PTH) monitoring, to document complete removal of hyperfunctioning parathyroid tissue (265). As the surgical management of patients with MEN2A and HPTH has evolved, option 3 has become the preferred procedure $(264,265)$. The management of HPTH in children is similar to that in adults; however, because of the rarity of the disease in youngsters there are little data on long-term outcomes.

A more difficult situation arises when HPTH develops subsequent to thyroidectomy. In this setting preoperative localization (with sestamibi scanning, US, or CT) should precede repeat neck exploration. Enlarged parathyroids should be excised with PTH monitoring; however, it may be difficult to identify all parathyroid glands, since some may be masked by scarring or were removed at prior thyroidectomy.

If a single large parathyroid gland and one or more normal size parathyroid glands are found, the enlarged parathyroid should be resected, and parathyroid function monitored by PTH immunoassay. If PTH levels are normal the operation can be terminated. If, however, there is evidence of persistent HPTH, the search for enlarged parathyroids should continue.

If a single large parathyroid gland is identified and the status of the remaining parathyroids is unknown, the large gland should be resected and parathyroid function monitored by PTH immunoassay. If there is evidence of normal parathyroid function the operation can be terminated. If there is no evidence of function a portion of the removed parathyroid should be grafted to a heteropic site.

If one enlarged parathyroid gland is identified and there is histological documentation that three glands have been re- moved previously, either a portion of the gland should be left in situ on a vascular pedicle, or the gland should be resected and slivers of it grafted to a heterotopic site. Alternatively, the gland could be resected and a portion cryopreserved for subsequent autografting. Currently, cryopreservation is practiced infrequently at a few medical centers (266). If the patient remains hyperparathyroid despite one or more reoperations, or the patient is not a candidate for parathyroidectomy, medical therapy with calcimimetics should be considered $(267,268)$.

\section{- RECOMMENDATION 42}

Patients in the ATA-H and ATA-MOD categories should be screened for HPTH at the time of screening for PHEO (by age 11 years in patients in the ATA-H category and by age 16 years in patients in the ATA-MOD category). Grade C Recommendation

\section{- RECOMMENDATION 43}

In patients with HPTH only visibly enlarged parathyroid glands should be resected. If all four glands are enlarged surgical options include subtotal parathyroidectomy with a piece of one gland left in situ on a vascular pedicle or total parathyroidectomy with a heterotopic autograft. Grade C Recommendation

\section{- RECOMMENDATION 44}

Patients who develop HPTH subsequent to thyroidectomy for MTC should have localization studies performed prior to repeat neck surgery. At reoperation all enlarged parathyroid glands should be removed, and parathyroids of normal size should be left in situ. If only one enlarged parathyroid gland is identified and there is histological documentation that three parathyroid glands have been removed previously, a portion of the enlarged gland should either be left in situ with an adequate blood supply or grafted to a heterotopic site. Grade C Recommendation

\section{[R] EVALUATION OF PATIENTS FOLLOWING THYROIDECTOMY (FIGURE 3)}

Postoperative staging is used to separate low-risk from high-risk patients with MTC. The AJCC staging system has been applied to patients with MTC (Table 5). The TNM system utilizes tumor size, extrathyroidal invasion, nodal metastasis, and distant spread of cancer. However, in patients with MTC the TNM classification lacks important prognostic factors such as gradations of age and postoperative serum Ctn levels. Also, the AJCC TNM classifications for MTC categorize lymph node metastases not by number but according to the location of nodes within (N1a) or without (N1b) the central neck, regardless of the number of lymph node metastases or involved compartments. The Task Force felt that the AJCC staging system should be modified to include four groups of lymph node metastases: 0, 1-10, 11-20, and > 20 (200,204).

It is important to evaluate patients in the postoperative period to determine if thyroidectomy has been curative. In a study of 124 patients having total thyroidectomy for tumors other than MTC, 121 (97\%) had no serum Ctn detectable by ICMA. In those with detectable Ctn levels, there was either residual thyroid tissue or ectopic secretion of $\mathrm{Ctn}$ from a nonthyroid malignancy (269). These data indicate that 


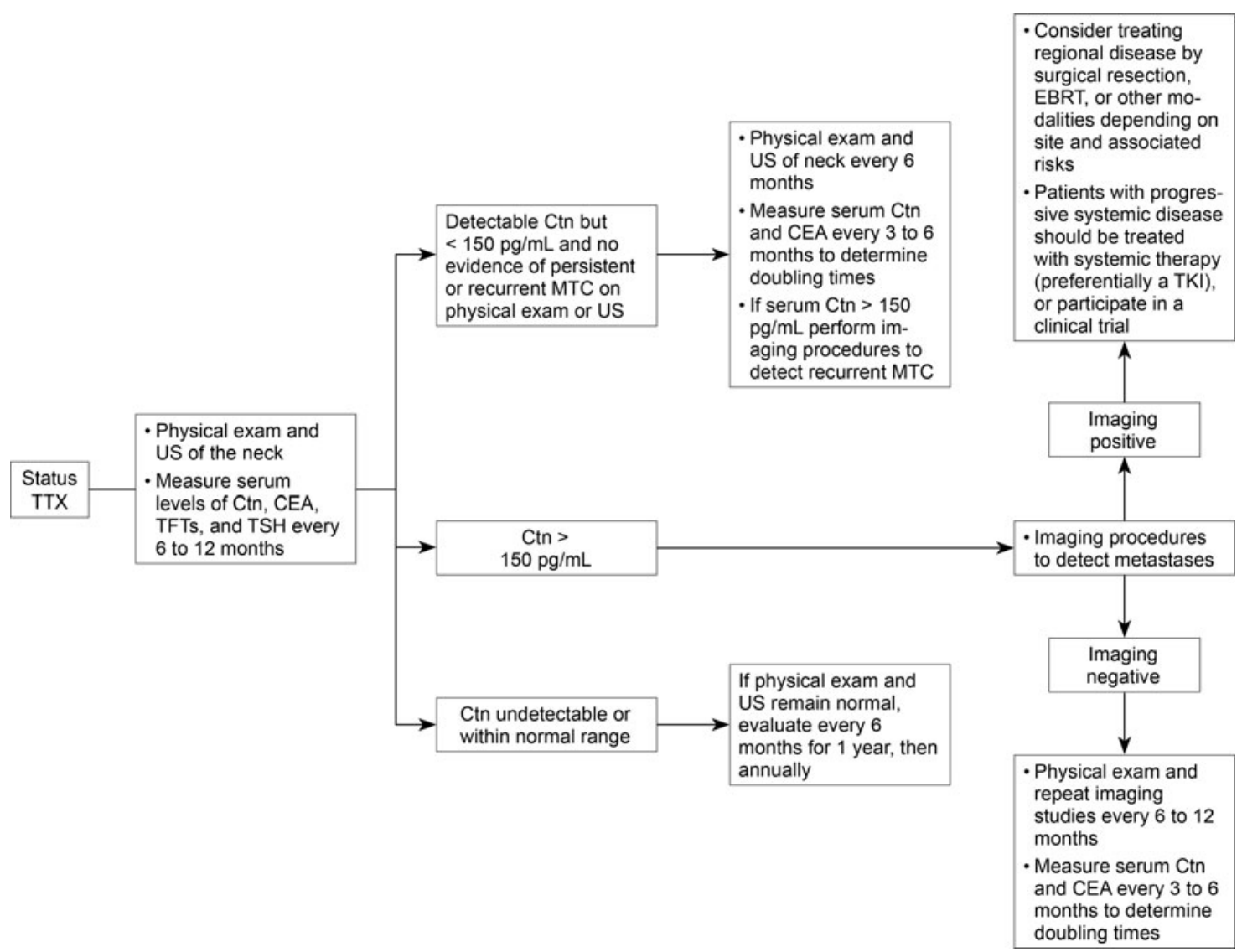

FIG. 3. Management of patients following thyroidectomy for persistent or recurrent medullary thyroid carcinoma. Ctn, calcitonin; CEA, carcinoembryonic antigen; EBRT, external beam radiotherapy; MTC, medullary thyroid carcinoma; TFTs, thyroid function tests; TSH, thyrotropin; TKI, tyrosine kinase inhibitor; TTX, total thyroidectomy; US, ultrasound.

postoperative serum Ctn levels should be undetectable following complete removal of thyroid tissue. In the same study 32 of 68 patients with MTC treated by total thyroidectomy had undetectable basal and stimulated serum Ctn levels postoperatively and appeared cured. Of the remaining 36 patients, 22 had pathologically increased basal serum Ctn levels and in 11 of them residual MTC tissue was identified postoperatively. Progressive increases in serum $\mathrm{Ctn}$ in the other 11 patients indicated tumor recurrence. Of the remaining 14 patients, nine had undetectable basal serum $\mathrm{Ctn}$ levels; however, in two patients serum $\mathrm{Ctn}$ levels increased to a pathological range with stimulation. The remaining five patients had measureable serum Ctn within the normal range, but in three patients there was a pathological increase in serum Ctn with stimulation. In 2 of the 14 patients there was evidence of recurrent MTC; however, there was no clinical or imaging evidence of recurrent disease in 12 of the 14 patients. Because of the short length of evaluation after thyroidectomy, it was impossible to determine the frequency with which metastatic MTC developed in the various groups of patients (269).

The normalization of serum Ctn levels postoperatively is associated with a favorable outcome; however, there has been controversy regarding how long it takes for the serum Ctn level to reach its nadir. Some clinical investigators have proposed that 3 months postoperatively is the optimal time to determine the nadir of serum Ctn levels $(270,271)$. Due to its prolonged half-life, serum levels of CEA may take even longer to reach a nadir. Some investigators consider a basal or stimulated serum Ctn level at or below the limits of the ICMA indicative of a curative thyroidectomy for MTC (272). Others propose that a thyroidectomy is curative if the postoperative stimulated serum Ctn level is less than $10 \mathrm{pg} / \mathrm{mL}(271,273)$. Using this latter criterion, 453 of 1430 patients in the Groupe d'Etude des Tumeurs à Calcitonine database treated by total thyroidectomy with or without lymph node dissection were considered to have residual disease at their last evaluation. Fifteen $(3.7 \%)$ of these patients met the criteria for recurrence because they had been considered cured (basal and pentagastrin stimulated serum Ctn less than $10 \mathrm{pg} / \mathrm{ML}) 6$ months after surgery (195).

In a study of 63 consecutive patients treated by total thyroidectomy for MTC, the postoperative basal serum Ctn level was less than $10 \mathrm{pg} / \mathrm{mL}$ in 35 patients and their 3- and 5-year relapse-free survival rates were $94 \%$ and $90 \%$, respectively, as opposed to $78 \%$ and $61 \%$ in patients with basal Ctn levels above $10 \mathrm{pg} / \mathrm{mL}$ (272). In patients with basal serum Ctn levels less than $150 \mathrm{pg} / \mathrm{mL}$ following thyroidectomy, persistent or recurrent disease is almost always confined to lymph nodes in the neck (272).

Detection of persistent or recurrent MTC should begin with neck US. In a study of 212 patients with various histological types of thyroid carcinoma additional sites of metastatic disease were detected by US but not physical examination in $21(20 \%)$ of 107 patients prior to primary thyroidectomy, in $9(32 \%)$ of 28 patients with persistent disease 
after thyroidectomy, and in $52(68 \%)$ of 77 patients with recurrent disease after thyroidectomy. The numbers for 61 patients with MTC were, respectively, $6(27 \%)$ of 22 patients, $7(70 \%)$ of 10 patients, and 17 (59\%) of 29 patients (274). FNA biopsy is useful in confirming the diagnosis of metastatic MTC, and measurement of Ctn levels in the FNA washout fluid may increase the specificity and sensitivity of the procedure $(182,183)$.

Imaging techniques to detect persistent or recurrent MTC include neck and chest CT, three-phase contrast-enhanced CT, contrast-enhanced MRI, and US of the liver, bone scintigraphy, MRI of the spine and pelvis, PET, and FDG-PET/ CT, and F-DOPA-PET/CT (188,275-280). The sensitivity of these tests in localizing metastatic disease ranges between $50 \%$ and $80 \%$, but it is likely to be significantly lower in the setting of modestly elevated serum Ctn values $(277,278)$.

In one study imaging procedures were performed in 55 consecutive patients with MTC and elevated serum levels of Ctn (median $1250 \mathrm{pg} / \mathrm{mL}$ ) and CEA (median $37 \mathrm{ng} / \mathrm{mL}$ ). Neck recurrences were demonstrated in 50 patients by US (56\%), CT (42\%), and FDG-PET/CT (32\%). Lung and mediastinal lymph node metastases were demonstrated in 55 patients by CT (35\% and $31 \%$, respectively) and by FDGPET/CT (15\% and 20\%, respectively). Liver metastases were demonstrated in 41 patients by MRI (49\%), CT (41\%), US (41\%), and FDG-PET/CT $(27 \%)$. Bone metastases were demonstrated in 55 patients by FDG-PET/CT (35\%), bone scintigraphy (40\%), and MRI (40\%). Bone scintigraphy was complementary with MRI for axial skeletal lesions but superior to MRI for the detection of peripheral bone lesions. The authors concluded that the most efficient imaging procedures for detecting MTC at various sites were US (neck), CT (chest), MRI (liver), and MRI and bone scintigraphy (axial skeleton) (188). Imaging with anti-CEA antibodies and scintigraphy with various tracers, such as somatostatin analogs, metaiodobenzylguanidine (MIBG), dimercaptosuccinic acid, and gastrin, are usually of low sensitivity (281-286).

In subsequent studies, however, FDG PET/CT and F-DOPA PET/CT proved superior to conventional imaging procedures in detecting metastases in patients with MTC (287-291). FDOPA PET/CT had a higher sensitivity, compared to FDG$\mathrm{PET} / \mathrm{CT}$, and seemed more important in assessing extent of disease. On the other hand, FDG-PET/CT correlated significantly with progressive disease. Survival was significantly lower in FDG-PET/CT-positive patients compared to FDGPET/CT-negative patients, and although the same was true for F-DOPA PET/CT-positive patients compared to those who were negative, the survival in patients with a positive FDGPET/CT was lower and independent of the F-DOPA-PET/CT result. Therefore, the two studies are complementary with FDOPA PET/CT having a higher sensitivity in detecting tumor load, and FDG-PET/CT more accurately identifying patients with progressive disease $(290,291)$. However, these imaging techniques with PET are expensive and selection criteria and a medico-economic evaluation are required before being used routinely in clinical practice.

\section{- RECOMMENDATION 45}

Clinicians should consider TNM classification, the number of lymph node metastases, and postoperative serum Ctn levels in predicting outcome and planning long-term follow-up of patients treated by thyroidectomy for MTC. Grade $\mathrm{C}$ recommendation

\section{- RECOMMENDATION 46}

Serum levels of Ctn and CEA, should be measured 3 months postoperatively, and if undetectable or within the normal range, they should be measured every 6 months for 1 year, and then yearly thereafter. Grade $\mathrm{C}$ Recommendation

\section{- RECOMMENDATION 47}

Patients with elevated postoperative serum Ctn levels less than $150 \mathrm{pg} / \mathrm{mL}$ should have a physical examination and US of the neck. If these studies are negative the patients should be followed with physical examinations, measurement of serum levels of Ctn and CEA, and US every 6 months. Grade C Recommendation

\section{- RECOMMENDATION 48}

If the postoperative serum Ctn level exceeds $150 \mathrm{pg} / \mathrm{mL}$ patients should be evaluated by imaging procedures, including neck US, chest CT, contrast-enhanced MRI or three-phase contrast-enhanced CT of the liver, and bone scintigraphy and MRI of the pelvis and axial skeleton. Grade C Recommendation

\section{[R-1] Measurement of doubling times of serum Ctn and CEA to determine rate of progression of MTC}

One can estimate the growth rate of MTC from sequential imaging studies using response evaluation criteria in solid tumors (RECIST) that document incremental increases in tumor size over time (292). One can also determine the MTC growth rate by measuring serum levels of Ctn or CEA over multiple time points to determine the rate at which each marker's value doubles (293-296). In a retrospective study of 65 patients treated by total thyroidectomy and bilateral lymph node dissection and then evaluated from 2.9 to 29.5 years after surgery, the 5- and 10-year survival rates in those with serum $\mathrm{Ctn}$ doubling times less than 6 months were $25 \%$ and $8 \%$, respectively, compared to $92 \%$ and $37 \%$ respectively in those with doubling times between 6 and 24 months (293). All patients with Ctn doubling times greater than 24 months were alive at the end of the study. The TNM stage, the European Organization for Research and Treatment of Cancer score, and Ctn doubling time were significant predictors of survival by univariate analysis, but only the Ctn doubling time was an independent predictor of survival by multivariate analysis. The Ctn doubling time was a better predictor of survival than the CEA doubling time (293). In another study of 55 consecutive patients with MTC the doubling times of $\mathrm{Ctn}$ and CEA were concordant in $80 \%$ of patients, such that when both markers were 25 months or less (16 of 17 patients) or 25 months or greater (12 of 14 patients), progressive disease was evident in $94 \%$ and $14 \%$, respectively (294). When the doubling times were discordant (nine patients) and either the Ctn doubling time or the CEA doubling time was 25 months or less, progressive disease was observed in five patients. The clinician should determine the doubling times of both markers (294). To calculate the doubling time of a tumor marker single exponentials are fitted to the tumor marker concentration by nonlinear least square regression. 
Reliable estimates are obtained using at least four data points over a minimum of 2 years; however, doubling times less than 6 months can be reliably estimated within the first 12 months postoperatively (293). The ATA provides a calculator to determine doubling times of serial serum $\mathrm{Ctn}$ and CEA measurements (www.thyroid.org/thyroid-physiciansprofessionals/calculators/thyroid-cancer-carcinoma).

\section{- RECOMMENDATION 49}

In patients with detectable serum levels of Ctn and CEA following thyroidectomy, the levels of the markers should be measured at least every 6 months to determine their doubling times. Grade B recommendation

\section{[S] TREATMENT OF PATIENTS WITH REGIONAL METASTATIC MTC}

\section{[S-1] Neck exploration}

In a recent study of patients with MTC nodal metastases began appearing at basal serum Ctn levels of $40 \mathrm{pg} / \mathrm{mL}$ in patients having initial thyroidectomy and $10 \mathrm{pg} / \mathrm{mL}$ in patients having reoperative surgery (reference range $<10 \mathrm{pg} / \mathrm{mL}$ ). In patients with lymph node metastases at the time of reoperation, distant metastases began appearing at preoperative basal serum Ctn levels above $150 \mathrm{pg} / \mathrm{mL}$, exceeded $50 \%$ at Ctn levels of $5000 \mathrm{pg} / \mathrm{mL}$, and were virtually always present when Ctn levels exceeded $20,000 \mathrm{pg} / \mathrm{mL}$ (206). In a recent study of patients with one to two or four sites of distant metastases, the median (and range) of serum Ctn was $1510 \mathrm{pg} / \mathrm{mL}$ (21$6000 \mathrm{pg} / \mathrm{mL}$ ) and $18,450 \mathrm{pg} / \mathrm{mL}(276-247,000 \mathrm{pg} / \mathrm{mL})$, respectively (294).

Patients with persistent or recurrent MTC localized to the neck following thyroidectomy are candidates for repeat neck operations. Several investigators have reported their experience with repeat neck surgery for persistent or recurrent regional MTC; however, there has been no clinical trial where patients have been staged and randomized to either reoperation or observation alone. In about one third of patients treated by repeat neck operations for persistent or recurrent MTC, the postoperative basal or stimulated serum $\mathrm{Ctn}$ levels are reduced to the "normal range"; however, they are rarely undetectable (297-300). Long-term outcomes in patients undergoing repeat neck operations have been fairly good, with excellent prevention of recurrence in the central neck. One group reported durable reductions in serum Ctn levels (297).

\section{- RECOMMENDATION 50}

Surgical resection of persistent or recurrent loco-regional MTC in patients without distant metastases should include compartmental dissection of image-positive or biopsypositive disease in the central (level VI) or lateral (levels II-V) neck compartments. Limited operative procedures, such as resection of only grossly metastatic lymph nodes, should be avoided unless there has been prior extensive surgery in a compartment. Grade C Recommendation

\section{[S-2] Role of postoperative radioiodine ablation}

Clinical investigators considered the possibility that RAI uptake in follicular cells might damage adjacent MTC cells by way of a "bystander effect" $(301,302)$. However, in a recent study of 293 patients treated by total thyroidectomy for
MTC, there was no difference in disease-specific survival in 61 patients who received postoperative RAI compared to 232 who did not. The authors concluded that RAI is not indicated following thyroidectomy for MTC (303). However, RAI should be considered in the rare patient whose regional or distant metastases contain MTC mixed with either PTC or FTC $(163,164)$.

\section{- RECOMMENDATION 51}

Postoperative RAI is not indicated following thyroidectomy for MTC; however, it should be considered in patients whose regional or distant metastases contain MTC mixed with either PTC or FTC. Grade E Recommendation

\section{[S-3] Adjunctive EBRT to the neck}

The benefits of EBRT adjuvant to thyroidectomy in patients with MTC are difficult to evaluate because there have been no prospective trials in which patients have been randomized to EBRT compared to observation alone (304-310). Generally, EBRT has been reserved for selected patients with a high likelihood of tumor recurrence following thyroidectomy, as determined by the operating surgeon and the radiation oncologist.

In a study of 51 patients with MTC who received adjuvant EBRT in doses ranging from 60 to $65 \mathrm{~Gy}$, the only significant variable for loco-regional recurrence-free survival on multivariate analysis was postoperative residual disease status ( $p=0.0005)$. The 5-year rate of local control was $100 \%$ in those without residual disease, $65 \%$ in those with microscopic residual disease, and $24 \%$ in those with gross residual disease (305). One of the largest studies of MTC involved 73 patients treated by total or near total thyroidectomy. Extraglandular extension was present in $56 \%$ of patients and $74 \%$ of patients had lymph node metastases. Patients were treated with EBRT $(n=46)$ ranging from 20 to $75.5 \mathrm{~Gy}$ (median $40 \mathrm{~Gy}$ ) or were observed $(n=27)$. Comparing treated to untreated patients, there was no difference in local or regional relapse-free rates (309).

In an analysis of the SEER data from 1998 to 2004, adjuvant EBRT showed no overall survival benefit in patients with MTC and positive lymph nodes (306). The intent of postoperative EBRT in patients with MTC, however, has not been to improve survival, but to achieve local control in those at high risk of regional recurrence. Several studies suggest that EBRT is effective in this regard. For example 34 patients at high risk for MTC recurrence following thyroidectomy were treated with a median total radiation dose of $60 \mathrm{~Gy}$ with conformal techniques. The 5-year loco-regional relapse-free survival rate was $87 \%$ and the disease-specific and overall survival rates were $62 \%$ and $56 \%$, respectively (307). In a recent study of 17 patients treated with adjuvant EBRT with a total dose of $60.8 \mathrm{~Gy}$, none of six patients who received adjuvant therapy developed recurrence in the radiated field. Five patients developed locoregional recurrence and were treated with a total dose of 59.4 Gy and a durable response was seen in three patients. The authors concluded that adjuvant EBRT might be effective in preventing loco-regional recurrence in selected patients (310).

Acute toxicity of EBRT includes skin erythema and desquamation, mucositis, esophagitis, and laryngeal edema. Due to odynophagia and dysphagia, some patients require a percutaneous gastrostomy (PEG) tube for nutrition. In most patients, the PEG tube is removed when acute toxicity has 
resolved, usually within 2-3 months after completion of EBRT. Late toxicities include skin hyperpigmentation, telangiectasia, hoarseness, osteoradionecrosis, trismus, arterial injury, and brain or central nerve injury (311). Xerostomia may also occur following EBRT although the parotid glands are better spared with the use of intensity-modulated radiation therapy (IMRT). Long-term esophageal stricture requiring dilatation or nutritional support or tracheal stenosis may also occur but are rare. Scarring and fibrosis occurring after EBRT may complicate subsequent surgery. Despite the potential acute and late toxicities, EBRT is fairly well tolerated (311).

Loco-regional control is a valid endpoint in patients with MTC, as progression in the cervical region can have a significant impact on quality of life. Adjuvant EBRT should be considered in patients with incompletely resected MTC or those at high risk for local recurrence; however, the potential benefits must be weighed against the probable acute and chronic toxicity associated with the therapy. Prior to initiating EBRT, surgeons should be sure that patients are not candidates for a repeat operation because the procedure will be more difficult technically following EBRT and will be associated with significant complications.

The typical postoperative dose, 60-66 Gy, delivered to the thyroid bed over 6 weeks using 4-6 MV photons, should eradicate microscopic disease. Gross residual disease should be treated to $70 \mathrm{~Gy}$ or higher for effective local control. Based on CT findings, IMRT should be used to treat MTC adjacent to the spinal cord. Dosimetric studies have demonstrated that dose escalation to the thyroid bed can be accomplished using IMRT while simultaneously sparing normal tissues. Imaging with CT, MRI, and PET ensures accurate target volume delineation, which must encompass the thyroid bed including the central compartment. The ipsilateral and contralateral compartments may also be encompassed within the target at the discretion of the radiation oncologist.

\section{- RECOMMENDATION 52}

Postoperative adjuvant EBRT to the neck and mediastinum should be considered in patients at high risk for local recurrence (microscopic or macroscopic residual MTC, extrathyroidal extension, or extensive lymph node metastases), and those at risk of airway obstruction. The potential benefits must be weighed against the acute and chronic toxicity associated with the therapy. Grade C Recommendation.

\section{[T] EVALUATION OF PATIENTS WITH DISTANT METASTASES (FIGURE 3)}

All patients with cancer, especially those in whom cancer is incurable, benefit from psychological support. Even though the presence of a somatic RET codon M918T mutation in a patient's tumor or rapid doubling times of serum $\mathrm{Ctn}$ and CEA levels are useful prognostic indicators, it is best to do nothing in asymptomatic patients with no detectable metastases. Once metastases appear, the clinician must decide which patients require therapy, balancing the often slow rate of tumor progression associated with a good quality of life, against the limited efficacy and potential toxicities of local and systemic therapies. Considering that metastatic MTC is incurable, the management goals are to provide loco-regional disease control, palliate symptoms of hormonal excess (such as diarrhea or Cushing's syndrome), palliate symptomatic metastases (such as pain or bone fracture), and control metastases that threaten life (such as bronchial obstruction or spinal cord compression).

\section{[T-1] Role of open or laparoscopic evaluation of the liver and selective venous catheterization with measurement of hepatic and peripheral vein stimulated Ctn levels}

Standard imaging procedures often fail to detect MTC metastases in patients with elevated serum Ctn levels following thyroidectomy. Techniques such as laparoscopic or open evaluation of the liver or sampling of the venous effluent of solid organs to detect elevated Ctn levels could document the presence of occult metastases. Patients shown to have distant metastases would not be candidates for extensive loco-regional surgery with curative intent. In 41 patients with elevated serum Ctn levels following thyroidectomy for MTC various localization procedures were performed: CT or MRI $(n=37)$, laparoscopic $(n=36)$ or open $(n=5)$ visual examination and biopsy of the liver, and selective venous catheterization, with measurement of hepatic and peripheral vein stimulated Ctn levels $(n=17)$ (312). Only laparoscopic or open evaluation and biopsy of the liver proved useful in the identification of occult metastatic disease. Liver metastases, identified in $8(19.5 \%)$ of 41 patients by laparoscopic $(n=7)$ or open evaluation, appeared as small white nodules less than $5 \mathrm{~mm}$ in size (312). CT scans identified metastatic disease in only one of the eight patients. Of 17 patients evaluated by hepatic vein sampling, 10 had hepatic vein/peripheral vein serum $\mathrm{Ctn}$ ratios greater than 1.3; however, only two of them had metastases confirmed by laparoscopy. Most asymptomatic patients with elevated serum Ctn levels and occult metastatic disease are not surgical candidates because natural history studies indicate that many of them have a relatively good prognosis, especially if the Ctn and CEA doubling times exceed 2 years (313-315).

\section{- RECOMMENDATION 53}

Systemic therapy should not be administered to patients who have increasing serum $\mathrm{Ctn}$ and CEA levels but no documented metastatic disease. Nor should systemic therapy be administered to patients with stable low-volume metastatic disease, as determined by imaging studies and serum $\mathrm{Ctn}$ and CEA doubling times greater than 2 years. Grade C Recommendation

\section{- RECOMMENDATION 54}

In patients with persistent or recurrent MTC following thyroidectomy, one should consider laparoscopic or open evaluation and biopsy of the liver to exclude occult metastases before subjecting them to a long and arduous repeat neck operation. Grade C Recommendation

\section{[U] DIAGNOSIS AND TREATMENT OF PATIENTS WITH CLINICALLY EVIDENT METASTATIC DISEASE}

\section{[U-1] Brain metastases}

Clinically apparent brain metastases occur in 1\%-5\% of patients with MTC. Börcek and associates found only 10 reported cases in a literature review; however, the prevalence is almost certainly underreported due to the infrequent use of 
central nervous system imaging in patients with advanced MTC (316). Patients with widespread metastases and neurological symptoms should be evaluated for the presence of brain metastases. Treatment of brain metastases by surgical resection, EBRT, or stereotaxic radiosurgery may provide local control and improve the quality of life; however, patients usually succumb from progressive systemic disease within a short time after the diagnosis of central nervous system metastases $(317,318)$.

\section{- RECOMMENDATION 55}

Brain imaging should be performed in patients with metastatic MTC and neurologic symptoms, including patients who are candidates for systemic therapy. Patients with isolated brain metastases are candidates for surgical resection or EBRT (including stereotactic radiosurgery). Whole-brain EBRT is indicated for multiple brain metastasis. Grade C Recommendation

\section{[U-2] Bone metastases}

The management of patients with bone metastases includes therapies such as vertebroplasty, surgical excision, thermoablation (radiofrequency or cryoablation), cement injection, and EBRT (319). Patients with acute symptoms from spinal cord compression require urgent treatment with corticosteroid administration and acute surgical stabilization (320). It is extremely uncommon for patients with bone metastases to be rendered free of disease; however, in one study $70 \%$ of patients treated with EBRT had significant pain reduction lasting for months (321). Treatment with either intravenous bisphosphonates (zoledronic acid or pamidronate) or the receptor activator of nuclear factor kappa-B ligand (RANKL) inhibitor, denosumab, in patients with thyroid cancer has been effective in relieving pain from bony metastases and in preventing or delaying the occurrence of other skeletal related events (322). Side effects of these potent antiresorptives include osteonecrosis of the jaw, atypical subtrochanteric fractures, and hypocalcemia $(323,324)$. There is little experience with studies of bisphosphonates in patients with MTC.

\section{- RECOMMENDATION 56}

Patients with spinal cord compression require urgent treatment with glucocorticoid therapy and surgical decompression. If patients are not candidates for surgery EBRT alone should be administered. Grade C Recommendation

\section{- RECOMMENDATION 57}

Patients with MTC who have fractures or impending fractures require treatment. Therapeutic options include surgery, thermoablation (radiofrequency or cryotherapy), cement injection, and EBRT. Grade C Recommendation

\section{- RECOMMENDATION 58}

Treatment with denosumab or bisphosphonates is recommended for patients with painful osseous metastases. Grade C Recommendation

\section{[U-3] Lung and mediastinal metastases}

Lung metastases are usually multiple and often associated with mediastinal lymph node metastases. It may be necessary to resect lung or mediastinal lesions causing local compression of an airway or bleeding. Other therapeutic options are EBRT or radiofrequency ablation. MTC invading the central airway may be amenable to laser therapy, photodynamic therapy, or airway stenting (325). Systemic therapy is indicated in patients with widespread metastases that are progressing.

\section{- RECOMMENDATION 59}

Surgical resection should be considered in patients with large solitary lung metastases. Radiofrequency ablation should be considered when the metastases are peripheral and small. Systemic therapy should be considered in patients with multiple metastases that are progressively increasing in size. Grade C Recommendation

\section{[U-4] Hepatic metastases}

Liver metastases occur in $45 \%$ of patients with advanced MTC. Treatment is indicated in patients with liver metastases that are large, increasing in size, or associated with symptoms such as diarrhea or pain. Single large metastases should be resected if possible. Liver metastases, however, are often multiple and disseminated throughout the parenchyma and are usually not amenable to surgery, percutaneous ethanol ablation, or radiofrequency ablation. In this situation the best treatment may be either chemoembolization or systemic therapy (326-329). In one study of chemoembolization in 12 patients with MTC metastatic to the liver a partial radiological response occurred in $42 \%$ and disease stabilization occurred in $42 \%$. Diarrhea improved in two (40\%) of five patients. Following a single cycle, the duration of partial responses and stabilizations exceeded 16 months. The extent of liver involvement was the main factor that influenced results. Partial responses were observed in patients with less than $30 \%$ of the liver involved and with individual metastases less than $30 \mathrm{~mm}$ in size. When disease progression occurred, patients could be offered repeat treatment, which often resulted in another partial tumor response, usually of shorter duration (326). In another study of 11 patients hepatic metastases were treated from one to nine times by chemoembolization. Symptomatic response was noted in all patients, but biochemical and radiological responses were noted in only six of them (328). It is important that patients have adequate renal and hepatic function and that they be carefully evaluated prior to treatment. One patient died during the first course of therapy due to the presence of an unexpected PHEO (328).

\section{- RECOMMENDATION 60}

Surgical resection should be considered in patients with large isolated hepatic metastases. Chemoembolization should be considered in patients with disseminated tumors less than $30 \mathrm{~mm}$ in size involving less than a third of the liver. Grade C Recommendation

\section{[U-5] Cutaneous metastases}

Patients with MTC rarely develop cutaneous metastases. Characteristically, they are erythematous, maculopapular eruptions, or tumor nodules that develop on the upper chest, neck, or scalp. The metastases are often located adjacent to a scar from a previous operation, or they develop as a component of widely metastatic disease. Percutaneous ethanol injection of cutaneous metastases may be useful (330). Skin metastases respond to treatment with EBRT, but the prognosis is poor because most patients succumb to their disease within a year after diagnosis (330). 


\section{- RECOMMENDATION 61}

If possible cutaneous metastases should be excised surgically. Multiple cutaneous lesions are best treated by EBRT or ethanol injection. Grade C Recommendation

\section{[U-6] Palliation of patients with advanced MTC}

Palliative surgery can be effective treatment for patients with metastatic disease. The metastases are frequently painful and resistant to medical treatment. Also, space-occupying metastases that cause acute spinal cord compression, or airway and esophageal obstruction (with coughing, dyspnea, and difficulty swallowing) can be improved by resection of tumor, EBRT, or the administration of systemic therapy. Tumor debulking is often effective in alleviating the diarrheal syndrome occurring in patients with advanced MTC.

\section{- RECOMMENDATION 62}

Palliative therapy, including surgery, EBRT, or systemic therapy, should be considered in patients with metastases causing pain, mechanical compression, or signs and symptoms of hormonal excess. Grade C Recommendation

\section{[V] SYSTEMIC THERAPY}

Single agent or combination cytotoxic chemotherapeutic regimens administered to patients with MTC are characterized by low response rates $(15 \%-20 \%)$ of short duration, although they may be indicated in selected patients. The most effective regimens are combination therapy with doxorubicin and another agent, or 5-fluorouracil and dacarbazine (331-333).

There is limited experience with radiolabeled molecules delivering high radiation dose to cancers. Response, survival, and long-term safety of systemic [ ${ }^{90}$ Y-DOTA]-TOC were evaluated in a phase II clinical trial of patients with advanced MTC, increasing serum Ctn levels, and tumor uptake on ${ }^{111} \mathrm{In}$ octreoscan (334). Of 31 patients, $18(58.1 \%)$ had a posttherapeutic prolongation of the serum Ctn doubling time of at least $100 \%$. Only $9(29 \%)$ of the 31 patients, however, experienced reduction of serum Ctn levels and were designated responders. The responders had a significantly longer median survival from the time of treatment compared to nonresponders, 74.5 months (range, 15.7-107 months) compared to 10.8 months (range, $1.4-85$ months; $p=0.02$ ). Thirteen percent of patients developed hematologic toxicities, and $23 \%$ developed renal toxicities. The degree of

${ }^{111}$ In-octreoscan tumor uptake was not associated with treatment response or improvement in survival (334).

The efficacy of pretargeted radio-immunotherapy with bispecific monoclonal antibody and ${ }^{131}$ I-labeled bivalent hapten has shown promising results in early studies of patients with advanced MTC; however, there have been no prospective, randomized trials comparing this therapy to other therapies or a placebo $(335,336)$. Treatment with ${ }^{131} \mathrm{I}-$ MIBG is generally regarded as ineffective in patients with MTC, although some reports have described partial tumor remission or stability $(337,338)$. At present treatment with radioisotope-based therapy should only be considered in the context of a clinical trial.

\section{- RECOMMENDATION 63}

The use of single agent or combinatorial cytotoxic chemotherapeutic regimens should not be administered as first-line therapy in patients with persistent or recurrent MTC given the low response rates and the advent of promising new treatment options. Grade D Recommendation

\section{- RECOMMENDATION 64}

Treatment with radiolabeled molecules or pretargeted radio-immunotherapy may be considered in selected patients, ideally in the setting of a well-designed clinical trial. Grade C Recommendation

\section{[V-1] The basis for targeted therapy with TKIs}

Germline RET mutations are present in virtually all patients with MEN2A and MEN2B. Approximately half of the patients with sporadic MTC have somatic RET mutations, and $18 \%-80 \%$ of patients without somatic RET mutations have somatic $R A S$ mutations. Also, vascular endothelial growth factor (VEGF) receptors (VEGFR-1 [FLT-1] and VEGFR-2 [FLK-1, KDR]) are often overexpressed in MTC, both in tumor cells and in the supporting vascular endothelium (339). Many agents that target VEGFR-2 kinase also target RET kinase. In recent years several TKIs (axitinib, cabozantinib, gefitinib, imatinib, motesanib, sorafenib, sunitinib, and vandetanib) have been evaluated in phase I, II, and III clinical trials of patients with advanced MTC (340350). In phase II clinical trials partial response rates ranged from $0 \%$ to $50 \%$, with a large number of patients demonstrating prolonged stable disease. On the basis of recently completed phase III clinical trials the U.S. Food and Drug Administration (FDA) and the European Medicines Agency (EMA) approved two orally administered TKIs, vandetanib (2011) and cabozantinib (2012), for the treatment of patients with advanced progressive MTC.

\section{[V-1-1] Clinical trials of vandetanib in patients with advanced MTC}

A phase II clinical trial with vandetanib, targeting the RET, EGFR, and VEGFR kinases, was evaluated at a maximal tolerated dose $(300 \mathrm{mg} / \mathrm{d})$ in 30 patients with hereditary MTC. Partial responses were observed in 10 patients, among whom six had a confirmed partial response, and another 16 patients had stable disease longer than 24 weeks (347). Another phase II trial with vandetanib $(100 \mathrm{mg} / \mathrm{d})$ included 19 patients with hereditary MTC. A partial response was observed in three patients and stable disease longer than 24 weeks was seen in another 10 patients. However, it was not clear whether there was a relationship between dose and efficacy or between dose and toxicity (348).

A prospective, randomized, double blind, phase III trial (National Clinical Trial [NCT]00322452) of 331 patients with symptomatic or progressing locally advanced or metastatic MTC compared PFS in patients treated with vandetanib (300 mg/d) or placebo (349). The median PFS was significantly prolonged from 19.3 months in the placebo arm to a predicted median of 30.5 months (median not yet reached) in the vandetanib arm (hazard ratio [HR], 0.46; $p<0.0001$ ). Partial responses were observed in $45 \%$ of patients treated with vandetanib, with a predicted median duration of response of 22 months. The improvement in quality of life, pain reduction, and diarrhea allowed several patients in the vandetanib arm to resume a normal social life. All subgroups of patients, with regard to tumor burden, progression rate, or 
symptoms, experienced significant PFS benefits from treatment. Also, PFS benefits were observed both in patients with a RET mutation and those without a RET mutation. In $41 \%$ of patients the RET status was unknown due to the inability to sequence all RET exons. Adverse events, including diarrhea, fatigue, rash and folliculitis, photosensitization, hypertension, and prolongation of the QTc interval, were mainly grade 1 or 2 . Twelve percent of patients receiving vandetanib discontinued treatment due to toxicity and $35 \%$ required dose reductions because of an adverse event (349). The FDA approved vandetanib in April 2011 with Risk Evaluation and Mitigation Strategies (REMS), and the EMA approved vandetanib in November 2011 for the treatment of aggressive and symptomatic MTC in patients with unresectable locally advanced or metastatic disease.

However, the EMA approval was conditional, and there was a request for the company marketing vandetanib to provide more evidence regarding benefit in patients with and without the RET mutation in their tumor.

\section{[V-1-2] Clinical trials of cabozantinib in patients with advanced MTC}

A phase I/II trial with cabozantinib, targeting the kinases of RET, c-MET, and VEGFR, included 35 evaluable MTC patients. Seventeen patients had a partial response, and in 10 of them a partial response was confirmed (344). Partial responses were observed regardless of somatic RET mutation status in both treatment-naïve patients and in those who had been treated previously with another kinase inhibitor, suggesting the absence of cross-resistance with other compounds. In a randomized, prospective phase III trial of 330 patients with progressive, metastatic, or locally advanced MTC (NCT00704730), treatment with cabozantinib (140 mg/ d) was compared to placebo (350). Median PFS was significantly improved from 4.0 months (placebo) compared to 11.2 months (cabozantininb) (HR, 0.28; $p<0.0001)$. Benefits in PFS were observed in all subgroups studied. The overall response rate was $28 \%$ (350). Side effects were significant and included diarrhea, abdominal discomfort, fatigue, hypertension, palmo-plantar erythrodysesthesia, and gastrointestinal fistulas. In fact, $16 \%$ of patients receiving cabozantinib discontinued treatment due to toxicity, and $79 \%$ required dose reductions because of an adverse event (350). A recent analysis demonstrated benefits of cabozantinib treatment in patients with either RET or RAS mutated tumors (351). On the basis of the results of the phase III clinical trial, the FDA and the EMA approved cabozantinib for the treatment of patients with advanced disease.

In the two phase III trials thus far completed, vandetanib and cabozantinib have shown the potential to provide high rates of disease control with durable responses and a highly significant improvement of PFS. However, the drugs have to be given daily and chronically to maintain tumor control. Short-term toxicity is significant, with dose reduction or treatment withdrawal in a significant proportion of patients. Also, there are few data on long-term toxicity and no data on overall survival. Currently, treatment with the drugs is indicated only in patients with significant tumor burden and documented tumor progression.

Patients treated with TKIs for advanced MTC require careful monitoring because they are at increased risk for developing hypothyroidism, characterized by elevated serum TSH levels despite normal serum levels of free triiodothy- ronine and thyroxine (340). Although the mechanism for this metabolic complication is unclear, it has recently been shown that the TKI sorafenib induces alteration in triiodothyronine and thyroxine clearance probably by inducing type 3deiodinase activity $(352,353)$.

Unresolved issues include the following:

1) Should vandetanib or cabozantinib be offered as firstline therapy? Is the FDA-approved starting dosage of $140 \mathrm{mg} / \mathrm{d}$ for cabozantinib too toxic? Is a starting dosage of $40-100 \mathrm{mg} / \mathrm{d}$ more reasonable? This latter dosage has been recommended in several clinical trials of prostate cancer since approval of the drug for the treatment of advanced MTC (354).

2) What are the mechanisms of resistance to the TKIs given that with time virtually all patients will cease to respond to the drugs?

3) Does treatment with either TKI improve overall survival? It must be noted that even when progression occurs, many patients have a long life expectancy and will have received several lines of treatment following therapy with either vandetanib or cabozantinib that may confound assessment of overall survival.

4) When should treatment with TKIs be terminated in patients with stable disease, or in patients who have a mixed response?

It is critical that we understand the mechanisms of resistance to these TKIs because without this information it will be impossible to develop trials of logical combinatorial therapies, either with two TKIs or a TKI and a conventional cytotoxic chemotherapeutic agent.

\section{- RECOMMENDATION 65}

In patients with significant tumor burden and symptomatic or progressive metastatic disease according to RECIST treatment with TKIs targeting both RET and VEGFR tyrosine kinases should be considered as systemic therapy. The TKIs vandetanib or cabozantinib can be used as single-agent first-line systemic therapy in patients with advanced progressive MTC. Grade A Recommendation

\section{[W] TREATMENT OF PATIENTS WITH HORMONALLY ACTIVE METASTASES}

Diarrhea is a common occurrence in patients with advanced MTC and markedly elevated serum Ctn levels. Rarely, the MTC cells inappropriately secrete corticotropinreleasing hormone $(\mathrm{CRH})$ or $\mathrm{ACTH}$.

\section{[W-1] Diarrhea}

Diarrhea occurs most frequently in patients with advanced disease and hepatic metastases. The diarrhea may be hypersecretory, due to enhanced gastrointestinal motility, or a combination of both $(355,356)$. The diarrhea can be debilitating both in terms of quality of life and nutrition. Patients with advanced MTC and diarrhea should be advised to avoid alcohol intake and to maintain a diet that limits high-fiber foods. The antimotility agents loperamide, diphenoxylate/ atropine, or codeine have minimal side effects and should be used as first-line therapy. Treatment with somatostatin analogs and debulking of large tumors has also been employed. 
Reports of somatostatin analog therapy for diarrhea have been small nonrandomized cohort studies with variable results, suggesting modestly improved symptoms in some patients $(357,358)$. In single-center studies the combination of somatostatin analogs with interferon-alpha has been reported to improve symptoms of flushing and diarrhea, although others have not confirmed these findings $(359,360)$. Local treatment of large hepatic metastases using selective arterial chemoembolization has also been reported in nonrandomized studies. In one study two of five patients with advanced MTC and diarrhea had an objective response to chemoembolization, and in a second study, all six patients with diarrhea improved following chemoembolization $(326,328)$.

\section{- RECOMMENDATION 66}

Patients with advanced MTC and diarrhea should be treated initially with antimotility agents. Alternative therapies include somatostatin analogs and local therapies such as surgery or chemoembolization. Grade C Recommendation

\section{[W-2] Ectopic Cushing's syndrome}

MTC can occasionally secrete high levels of bioactive hormones other than $\mathrm{Ctn}$. The most common ectopic hormones, CRH or ACTH, can cause Cushing's syndrome. MTC is reported to account for up to $1 \%-3 \%$ of all cases of ectopic Cushing's syndrome $(361,362)$. Control of elevated cortisol levels, and the associated clinical manifestations, may be achieved by debulking of large hepatic metastases (surgery or chemoembolization); by medical therapy using ketoconazole, mifepristone, aminoglutethimide (where available), metyrapone, or mitotane; or by bilateral adrenalectomy (363). The development of Cushing's syndrome in patients with advanced MTC is associated with poor survival; however, because the syndrome can be severe and debilitating, treatment should be considered even in the setting of widely metastatic MTC. It is important to treat hypokalemia, hypertension, diabetes, and gastritis in patients with ectopic Cushing's syndrome. It is of interest that there have been three recent reports of reversal of Cushing's syndrome following the administration of vandetanib to patients with advanced MTC, which may provide another therapeutic option $(364,365)$.

\section{- RECOMMENDATION 67}

Patients with metastatic MTC and Cushing's syndrome due to ectopic production of ACTH or CRH are often markedly debilitated and should be treated despite their poor prognosis. Treatment options include medical therapy with ketoconazole, mifepristone, aminoglutethimide, metyrapone, or mitotane. In cases refractory to medical treatment bilateral adrenalectomy is an option. Grade C Recommendation

\section{EPILOG}

The revised ATA Management Guidelines for Medullary Thyroid Carcinoma were written to provide clinicians with direction in the management of patients with sporadic and hereditary MTC. Despite significant advances in the diagnosis and treatment of patients with MTC, much remains to be done. In patients with advanced MTC there is a critical need for more effective therapies. The TKIs vandetanib and cabozantinib have increased PFS compared to placebo in patients with advanced MTC; however, with time the MTC becomes resistant to these drugs and the disease progresses. Although new molecular targeted therapeutics will be evaluated in clinical trials, it is unlikely that a single compound will be curative. Hopefully, preclinical studies of tumor cells and animal models of MTC, and especially molecular analysis of tumor tissues collected from patients on TKI clinical trials, will lead to an understanding of the mechanisms of tumor resistance, and thereby the development of effective combinatorial therapies.

\section{ACKNOWLEDGMENTS}

The ATA Guidelines Task Force would like to thank and acknowledge those who were of great help during the development of the revised guidelines: Ms. Bobbi Smith, Executive Director of the ATA, and the staff at the ATA, for their continuing involvement and support during the preparation of the manuscript; Ms. Alicia A. Livinski, Biomedical Librarian, National Institutes of Health Library for assistance with the manuscript and bibliography; Dr. Susan Shaw DeVesa, Biostatistics Branch, National Cancer Institute, for help in calculating the incidence of MEN2A and of MEN2B using the Surveillance, Epidemiology, and End Results (SEER) Stat version 8.1.2 Rate Session. Access the SEER 18 database at www.seer.cancer.gov). Incidence - SEER 18 Regs Research Data + Hurricane Katrina Impacted Louisiana Cases, Nov 2012 Sub (2000-2010) < Katrina/Rita Population Adjustment $>$ - Linked To County Attributes - Total U.S., 1969-2011 Counties, National Cancer Institute, DCCPS, Surveillance Research Program, Surveillance Systems Branch, released April 2013, based on the November 2012 submission. The Thyroid Cancer Survivors' Association, Inc., (ThyCa) for contributing an unrestricted educational grant towards the development of the Revised American Thyroid Association Guidelines for the Management of Medullary Thyroid Carcinoma. The American Thyroid Association provided the remainder of the funding. There was no commercial support.

\section{AUTHOR DISCLOSURE STATEMENT}

Rosella Elisei, (Consultant and/or Speakers Bureau: AstraZeneca, Bayer, Exelixis, and Genzyme); Karin FrankRaue, (Honorarium: Sobi); Furio Pacini, (Speakers Bureau: Bayer and Genzyme); Bruce Robinson, (Consultant: AstraZeneca, Bayer, and Eisai); Martin Schumberger, (Grant or Research Support: AstraZeneca, Bayer, Sobi-Exelixis); Manisha Shah (Grant or Research Support: Bayer, Eisai, and Exelixis); and Steve Waguespeck (Consultant: Novo Nordisk). These authors have nothing to disclose: Douglas Evans, Henning Dralle, Nancy Lee, Andreas Machens, Jeffrey Moley, and Samuel Wells.

\section{REFERENCES}

1. Cancer of the Thyroid Invasive: Trends in SEER Incidence and U.S. Mortality Using the Joinpoint Regression Program, 1975-2011(SEER) Stat version 8.1.2 Rate Session. (Access the SEER 18 database at www.seer .cancer.gov). Incidence - SEER 18 Regs Research Data + Hurricane Katrina Impacted Louisiana Cases, Nov 
2012 Sub $(2000-2010)<$ Katrina/Rita Population Adjustment > - Linked To County Attributes - Total U.S., 19692011 Counties, National Cancer Institute, DCCPS, Surveillance Research Program, Surveillance Systems Branch, released April 2013, based on the November 2012 submission.

2. Brandi ML, Gagel RF, Angeli A, Bilezikian JP, BeckPeccoz P, Bordi C, Conte-Devolx B, Falchetti A, Gheri RG, Libroia A, Lips CJ, Lombardi G, Mannelli M, Pacini F, Ponder BA, Raue F, Skogseid B, Tamburrano G, Thakker RV, Thompson NW, Tomassetti P, Tonelli F, Wells SA Jr, Marx SJ 2001 Guidelines for diagnosis and therapy of MEN type 1 and type 2. J Clin Endocrinol Metab 86:5658-5671.

3. Tuttle RM, Ball DW, Byrd D, Daniels GH, Dilawari RA, Doherty GM, Duh QY, Ehya H, Farrar WB, Haddad RI, Kandeel F, Kloos RT, Kopp P, Lamonica DM, Loree TR, Lydiatt WM, McCaffrey J, Olson JA Jr, Parks L, Ridge JA, Shah JP, Sherman SI, Sturgeon C, Waguespack SG, Wang TN, Wirth LJ 2010 Medullary carcinoma. J Natl Compr Canc Netw 8:512-530.

4. Chen H, Sippel RS, O'Dorisio MS, Vinik AI, Lloyd RV, Pacak K 2010 The North American Neuroendocrine Tumor Society consensus guideline for the diagnosis and management of neuroendocrine tumors: pheochromocytoma, paraganglioma, and medullary thyroid cancer. Pancreas 39:775-783.

5. Kloos RT, Eng C, Evans DB, Francis GL, Gagel RF, Gharib H, Moley JF, Pacini F, Ringel MD, Schlumberger M, Wells SA Jr 2009 Medullary thyroid cancer: management guidelines ofthe American Thyroid Association. Thyroid 19:565-612.

6. Jaquet J 1906 Ein Fall von metastasierenden Amyloidtumoren (Lymphosarkom). Virchows Arch Pathol Anat Physiol 185:251-268.

7. Hazard JB, Hawk WA, Crile G Jr 1959 Medullary (solid) carcinoma of the thyroid; a clinicopathologic entity. J Clin Endocrinol Metab 19:152-161.

8. Williams ED 1966 Histogenesis of medullary carcinoma of the thyroid. J Clin Pathol 19:114-118.

9. Tashjian AH Jr, Melvin EW 1968 Medullary carcinoma of the thyroid gland. Studies of thyrocalcitonin in plasma and tumor extracts. N Engl J Med 279:279-283.

10. Wells SA Jr, Baylin SB, Linehan WM, Farrell RE, Cox EB, Cooper CW 1978 Provocative agents and the diagnosis of medullary carcinoma of the thyroid gland. Ann Surg 188:139-141.

11. Pachnis V, Mankoo B, Costantini F 1993 Expression of the c-ret proto-oncogene during mouse embryogenesis. Development 119:1005-1017.

12. Zordan P, Tavella S, Brizzolara A, Biticchi R, Ceccherini I, Garofalo S, Ravazzolo R, Bocciardi R 2006 The immediate upstream sequence of the mouse Ret gene controls tissue-specific expression in transgenic mice. Int $\mathbf{J}$ Mol Med 18:601-608.

13. Takahashi M, Ritz J, Cooper GM 1985 Activation of a novel human transforming gene, ret, by DNA rearrangement. Cell 42:581-588.

14. Donis-Keller H, Dou S, Chi D, Carlson KM, Toshima K, Lairmore TC, Howe JR, Moley JF, Goodfellow P, Wells SA Jr 1993 Mutations in the RET proto-oncogene are associated with MEN 2A and FMTC. Hum Mol Genet 2:851-856.

15. Mulligan LM, Kwok JB, Healey CS, Elsdon MJ, Eng C, Gardner E, Love DR, Mole SE, Moore JK, Papi L, et al.
1993 Germ-line mutations of the RET proto-oncogene in multiple endocrine neoplasia type 2A. Nature 363:458460.

16. Carlson KM, Dou S, Chi D, Scavarda N, Toshima K, Jackson CE, Wells SA Jr, Goodfellow PJ, Donis-Keller H 1994 Single missense mutation in the tyrosine kinase catalytic domain of the RET protooncogene is associated with multiple endocrine neoplasia type 2B. Proc Natl Acad Sci U S A 91:1579-1583.

17. Hofstra RM, Landsvater RM, Ceccherini I, Stulp RP, Stelwagen T, Luo Y, Pasini B, Hoppener JW, van Amstel HK, Romeo G, et al. 1994 A mutation in the RET protooncogene associated with multiple endocrine neoplasia type $2 \mathrm{~B}$ and sporadic medullary thyroid carcinoma. Nature 367:375-376.

18. Eng C, Smith DP, Mulligan LM, Nagai MA, Healey CS, Ponder MA, Gardner E, Scheumann GF, Jackson CE, Tunnacliffe A, et al. 1994 Point mutation within the tyrosine kinase domain of the RET proto-oncogene in multiple endocrine neoplasia type $2 \mathrm{~B}$ and related sporadic tumours. Hum Mol Genet 3:237-241.

19. Marsh DJ, Learoyd DL, Andrew SD, Krishnan L, Pojer R, Richardson AL, Delbridge L, Eng C, Robinson BG 1996 Somatic mutations in the RET proto-oncogene in sporadic medullary thyroid carcinoma. Clin Endocrinol (Oxf) 44:249-257.

20. Moura MM, Cavaco BM, Pinto AE, Leite V 2011 High prevalence of RAS mutations in RET-negative sporadic medullary thyroid carcinomas. J Clin Endocrinol Metab 96:E863- E868.

21. Boichard A, Croux L, Al Ghuzlan A, Broutin S, Dupuy C, Leboulleux S, Schlumberger M, Bidart JM, Lacroix L 2012 Somatic RAS mutations occur in a large proportion of sporadic RET-negative medullary thyroid carcinomas and extend to a previously unidentified exon. J Clin Endocrinol Metab 97:E2031-E2035.

22. Ciampi R, Mian C, Fugazzola L, Cosci B, Romei C, Barollo S, Cirello V, Bottici V, Marconcini G, Rosa PM, Borrello MG, Basolo F, Ugolini C, Materazzi G, Pinchera A, Elisei R 2013 Evidence of a low prevalence of RAS mutations in a large medullary thyroid cancer series. Thyroid 23:50-57.

23. Agrawal N, Jiao Y, Sausen M, Leary R, Bettegowda C, Roberts NJ, Bhan S, Ho AS, Khan Z, Bishop J, Westra WH, Wood LD, Hruban RH, Tufano RP, Robinson B, Dralle H, Toledo SP, Toledo RA, Morris LG, Ghossein RA, Fagin JA, Chan TA, Velculescu VE, Vogelstein B, Kinzler KW, Papadopoulos N, Nelkin BD, Ball DW 2013 Exomic sequencing of medullary thyroid cancer reveals dominant and mutually exclusive oncogenic mutations in RET and RAS. J Clin Endocrinol Metab 98:E364-E369.

24. Schilling T, Burck J, Sinn HP, Clemens A, Otto HF, Hoppner W, Herfarth C, Ziegler R, Schwab M, Raue F 2001 Prognostic value of codon 918 (ATG $\rightarrow$ ACG) RET proto- oncogene mutations in sporadic medullary thyroid carcinoma. Int J Cancer 95:62-66.

25. Elisei R, Cosci B, Romei C, Bottici V, Renzini G, Molinaro E, Agate L, Vivaldi A, Faviana P, Basolo F, Miccoli P, Berti P, Pacini F, Pinchera A 2008 Prognostic significance of somatic RET oncogene mutations in sporadic medullary thyroid cancer: a 10-year follow-up study. J Clin Endocrinol Metab 93:682-687.

26. Romei C, Ugolini C, Cosci B, Torregrossa L, Vivaldi A, Ciampi R, Tacito A, Basolo F, Materazzi G, Miccoli P, 
Vitti P, Pinchera A, Elisei R 2012 Low prevalence of the somatic M918T RET mutation in micro-medullary thyroid cancer. Thyroid 22:476-481.

27. Santoro M, Melillo RM, Fusco A 2006 RET/PTC activation in papillary thyroid carcinoma: European Journal of Endocrinology Prize Lecture. Eur J Endocrinol 155: 645-653.

28. Kohno T, Ichikawa H, Totoki Y, Yasuda K, Hiramoto M, Nammo T, Sakamoto H, Tsuta K, Furuta K, Shimada Y, Iwakawa R, Ogiwara H, Oike T, Enari M, Schetter AJ, Okayama H, Haugen A, Skaug V, Chiku S, Yamanaka I, Arai Y, Watanabe S, Sekine I, Ogawa S, Harris CC, Tsuda H, Yoshida T, Yokota J, Shibata T 2012 KIF5B-RET fusions in lung adenocarcinoma. Nat Med 18:375-377.

29. Ballerini P, Struski S, Cresson C, Prade N, Toujani S, Deswarte C, Dobbelstein S, Petit A, Lapillonne H, Gautier EF, Demur C, Lippert E, Pages P, Mansat-De Mas V, Donadieu J, Huguet F, Dastugue N, Broccardo C, Perot C, Delabesse E 2012 RET fusion genes are associated with chronic myelomonocytic leukemia and enhance monocytic differentiation. Leukemia 26:2384-2389.

30. Attie T, Pelet A, Edery P, Eng C, Mulligan LM, Amiel J, Boutrand L, Beldjord C, Nihoul-Fekete C, Munnich A, et al. 1995 Diversity of RET proto-oncogene mutations in familial and sporadic Hirschsprung disease. Hum Mol Genet 4:1381-1386.

31. Amiel J, Sproat-Emison E, Garcia-Barcelo M, Lantieri F, Burzynski G, Borrego S, Pelet A, Arnold S, Miao X, Griseri P, Brooks AS, Antinolo G, de Pontual L, ClementZiza M, Munnich A, Kashuk C, West K, Wong KK, Lyonnet S, Chakravarti A, Tam PK, Ceccherini I, Hofstra RM, Fernandez R; Hirschsprung Disease Consortium 2008 Hirschsprung disease, associated syndromes and genetics: a review. J Med Genet 45:1-14.

32. Margraf RL, Crockett DK, Krautscheid PM, Seamons R, Calderon FR, Wittwer CT, Mao R 2009 Multiple endocrine neoplasia type 2 RET protooncogene database: repository of MEN2-associated RET sequence variation and reference for genotype/phenotype correlations. Hum Mutat 30:548-556.

33. Leboulleux S, Baudin E, Travagli JP, Schlumberger M 2004 Medullary thyroid carcinoma. Clin Endocrinol 61: 299-310.

34. Machens A, Hinze R, Thomusch O, Dralle H 2002 Pattern of nodal metastasis for primary and reoperative thyroid cancer. World J Surg 26:22-28.

35. Moley JF 2010 Medullary thyroid carcinoma: management of lymph node metastases. J Natl Compr Canc Netw 8:549-556.

36. Kebebew E, Ituarte PH, Siperstein AE, Duh QY, Clark OH 2000 Medullary thyroid carcinoma: clinical characteristics, treatment, prognostic factors, and a comparison of staging systems. Cancer 88:1139-1148.

37. Modigliani E, Cohen R, Campos JM, Conte-Devolx B, Maes B, Boneu A, Schlumberger M, Bigorgne JC, Dumontier P, Leclerc L, Corcuff B, Guilhem I 1998 Prognostic factors for survival and for biochemical cure in medullary thyroid carcinoma: results in 899 patients. The GETC Study Group. Groupe d'etude des tumeurs a calcitonine. Clin Endocrinol (Oxf) 48:265-273.

38. Kebebew E, Greenspan FS, Clark OH, Woeber KA, Grunwell J 2005 Extent of disease and practice patterns for medullary thyroid cancer. J Am Coll Surg 200:890 896.
39. Roman S, Lin R, Sosa JA 2006 Prognosis of medullary thyroid carcinoma: demographic, clinical, and pathologic predictors of survival in 1252 cases. Cancer 107:21342142.

40. Steiner AL, Goodman AD, Powers SR 1968 Study of a kindred with pheochromocytoma, medullary thyroid carcinoma, hyperparathyroidism and Cushing's disease: multiple endocrine neoplasia, type 2. Medicine (Baltimore) 47:371-409.

41. Wermer P 1954 Genetic aspects of adenomatosis of endocrine glands. Am J Med 16:363-371.

42. Telenius-Berg M, Berg B, Hamberger B, Tibblin S, Tisell LE, Ysander L, Welander G 1984 Impact of screening on prognosis in the multiple endocrine neoplasia type 2 syndromes: natural history and treatment results in 105 patients. Henry Ford Hosp Med J 32:225-231.

43. Gagel RF, Levy ML, Donovan DT, Alford BR, Wheeler T, Tschen JA 1989 Multiple endocrine neoplasia type 2a associated with cutaneous lichen amyloidosis. Ann Intern Med 111:802-806.

44. Borst MJ, VanCamp JM, Peacock ML, Decker RA 1995 Mutational analysis of multiple endocrine neoplasia type 2A associated with Hirschsprung's disease. Surgery 117: 386-391.

45. Williams ED, Pollock DJ 1966 Multiple mucosal neuromata with endocrine tumours: a syndrome allied to von Recklinghausen's disease. J Pathol Bacteriol 91:71-80.

46. Schimke RN, Hartmann WH, Prout TE, Rimoin DL 1968 Syndrome of bilateral pheochromocytoma, medullary thyroid carcinoma and multiple neuromas. A possible regulatory defect in the differentiation of chromaffin tissue. N Engl J Med 279:1-7.

47. Gorlin RJ, Sedano HO, Vickers RA, Cervenka J 1968 Multiple mucosal neuromas, pheochromocytoma and medullary carcinoma of the thyroid-a syndrome. Cancer 22:293-299.

48. Farndon JR, Leight GS, Dilley WG, Baylin SB, Smallridge RC, Harrison TS, Wells SA Jr 1986 Familial medullary thyroid carcinoma without associated endocrinopathies: a distinct clinical entity. Br J Surg 73: 278-281.

49. Eng C, Clayton D, Schuffenecker I, Lenoir G, Cote G, Gagel RF, van Amstel HK, Lips CJ, Nishisho I, Takai SI, Marsh DJ, Robinson BG, Frank-Raue K, Raue F, Xue F, Noll WW, Romei C, Pacini F, Fink M, Niederle B, Zedenius J, Nordenskjold M, Komminoth P, Hendy GN, Mulligan LM, et al. 1996 The relationship between specific RET proto-oncogene mutations and disease phenotype in multiple endocrine neoplasia type 2. International RET mutation consortium analysis. JAMA 276:15751579.

50. Moers AM, Landsvater RM, Schaap C, Jansen-Schillhorn van Veen JM, de Valk IA, Blijham GH, Hoppener JW, Vroom TM, van Amstel HK, Lips CJ 1996 Familial medullary thyroid carcinoma: not a distinct entity? Genotype-phenotype correlation in a large family. Am J Med 101:635-641.

51. Da Silva AM, Maciel RM, Da Silva MR, Toledo SR, De Carvalho MB, Cerutti JM 2003 A novel germ-line point mutation in RET exon 8 (Gly(533)Cys) in a large kindred with familial medullary thyroid carcinoma. J Clin Endocrinol Metab 88:5438-5443.

52. Kaldrymides P, Mytakidis N, Anagnostopoulos T, Vassiliou M, Tertipi A, Zahariou M, Rampias T, Koutsodontis 
G, Konstantopoulou I, Ladopoulou A, Bei T, Yannoukakos D 2006 A rare RET gene exon 8 mutation is found in two Greek kindreds with familial medullary thyroid carcinoma: implications for screening. Clin Endocrinol (Oxf) 64:561-566.

53. Oliveira MN, Hemerly JP, Bastos AU, Tamanaha R, Latini FR, Camacho CP, Impellizzeri A, Maciel RM, Cerutti JM 2011 The RET p.G533C mutation confers predisposition to multiple endocrine neoplasia type $2 \mathrm{~A}$ in a Brazilian kindred and is able to induce a malignant phenotype in vitro and in vivo. Thyroid 21:975-985.

54. Peppa M, Boutati E, Kamakari S, Pikounis V, Peros G, Panayiotides IG, Economopoulos T, Raptis SA, Hadjidakis D 2008 Multiple endocrine neoplasia type 2A in two families with the familial medullary thyroid carcinoma associated G533C mutation of the RET proto-oncogene. Eur J Endocrinol 159:767-771.

55. Bethanis S, Koutsodontis G, Palouka T, Avgoustis C, Yannoukakos D, Bei T, Papadopoulos S, Linos D, Tsagarakis S 2007 A newly detected mutation of the RET protooncogene in exon 8 as a cause of multiple endocrine neoplasia type 2A. Hormones (Athens) 6:152-156.

56. Castro MR, Thomas BC, Richards ML, Zhang J, Morris JC 2013 Multiple endocrine neoplasia type 2A due to an exon 8 (G533C) mutation in a large North American kindred. Thyroid 23:1547-1552.

57. Siggelkow H, Melzer A, Nolte W, Karsten K, Hoppner W, Hufner M 2001 Presentation of a kindred with familial medullary thyroid carcinoma and Cys611Phe mutation of the RET proto-oncogene demonstrating low grade malignancy. Eur J Endocrinol 144:467-473.

58. Boccia LM, Green JS, Joyce C, Eng C, Taylor SA, Mulligan LM 1997 Mutation of RET codon 768 is associated with the FMTC phenotype. Clin Genet 51:81-85.

59. Jimenez C, Dang GT, Schultz PN, El-Naggar A, Shapiro $\mathrm{S}$, Barnes EA, Evans DB, Vassilopoulou-Sellin R, Gagel RF, Cote GJ, Hoff AO 2004 A novel point mutation of the RET protooncogene involving the second intracellular tyrosine kinase domain in a family with medullary thyroid carcinoma. J Clin Endocrinol Metab 89:3521-3526.

60. Raue F, Frank-Raue K 2012 Genotype-phenotype correlation in multiple endocrine neoplasia type 2. Clinics (Sao Paulo) 67(Suppl 1):69-75.

61. Imai T, Uchino S, Okamoto T, Suzuki S, Kosugi S, Kikumori T, Sakurai A; MEN Consortium of Japan 2013 High penetrance of pheochromocytoma in multiple endocrine neoplasia 2 caused by germ line RET codon 634 mutation in Japanese patients. Eur J Endocrinol 168:683687.

62. Frank-Raue K, Rybicki LA, Erlic Z, Schweizer H, Winter A, Milos I, Toledo SP, Toledo RA, Tavares MR, Alevizaki M, Mian C, Siggelkow H, Hufner M, Wohllk N, Opocher G, Dvorakova S, Bendlova B, Czetwertynska M, Skasko E, Barontini M, Sanso G, Vorlander C, Maia AL, Patocs A, Links TP, de Groot JW, Kerstens MN, Valk GD, Miehle K, Musholt TJ, Biarnes J, Damjanovic S, Muresan M, Wuster C, Fassnacht M, Peczkowska M, Fauth C, Golcher H, Walter MA, Pichl J, Raue F, Eng C, Neumann HP; International RET Exon 10 Consortium 2011 Risk profiles and penetrance estimations in multiple endocrine neoplasia type $2 \mathrm{~A}$ caused by germline RET mutations located in exon 10. Hum Mutat 32:51-58.

63. Lairmore TC, Ball DW, Baylin SB, Wells SA Jr 1993 Management of pheochromocytomas in patients with multiple endocrine neoplasia type 2 syndromes. Ann Surg 217:595-601; discussion 601-603.

64. Lips CJ, Landsvater RM, Hoppener JW, Geerdink RA, Blijham G, van Veen JM, van Gils AP, de Wit MJ, Zewald RA, Berends MJ, et al. 1994 Clinical screening as compared with DNA analysis in families with multiple endocrine neoplasia type 2A. N Engl J Med 331:828-835.

65. Herfarth KK, Bartsch D, Doherty GM, Wells SA Jr, Lairmore TC 1996 Surgical management of hyperparathyroidism in patients with multiple endocrine neoplasia type 2A. Surgery 120:966-973; discussion 973-974.

66. Tanaka A, Arita K, Lai-Cheong JE, Palisson F, Hide M, McGrath JA 2009 New insight into mechanisms of pruritus from molecular studies on familial primary localized cutaneous amyloidosis. Brit J Derm 161:1217-1224.

67. Verga U, Fugazzola L, Cambiaghi S, Pritelli C, Alessi E, Cortelazzi D, Gangi E, Beck- Peccoz P 2003 Frequent association between MEN 2A and CLA. Clin Endocrinol (Oxf) 59:156-161.

68. Ceccherini I, Romei C, Barone V, Pacini F, Martino E, Loviselli A, Pinchera A, Romeo G 1994 Identification of the Cys634 $\rightarrow$ Tyr mutation of the RET proto-oncogene in a pedigree with multiple endocrine neoplasia type $2 \mathrm{~A}$ and localized CLA. J Endocrinol Invest 17:201-204.

69. Rothberg AE, Raymond VM, Gruber SB, Sisson J 2009 Familial medullary thyroid carcinoma associated with cutaneous lichen amyloidosis. Thyroid 19:651-655.

70. Mulligan LM, Eng C, Attie T, Lyonnet S, Marsh DJ, Hyland VJ, Robinson BG, Frilling A, Verellen-Dumoulin C, Safar A, et al. 1994 Diverse phenotypes associated with exon 10 mutations of the RET proto-oncogene. Hum Mol Genet 3:2163-2167.

71. Mulligan LM, Ponder BA 1995 Genetic basis of endocrine disease: multiple endocrine neoplasia type 2. J Clin Endocrinol Metab 80:1989-1995.

72. Verdy M, Weber AM, Roy CC, Morin CL, Cadotte M, Brochu P 1982 Hirschsprung's disease in a family with multiple endocrine neoplasia type 2. J Pediatr Gastroenterol Nutr 1:603-607.

73. Decker RA, Peacock ML 1998 Occurrence of MEN 2a in familial Hirschsprung's disease: a new indication for genetic testing of the RET proto-oncogene. J Pediatr Surg 33:207-214.

74. Sijmons RH, Hofstra RM, Wijburg FA, Links TP, Zwierstra RP, Vermey A, Aronson DC, Tan-Sindhunata G, Brouwers-Smalbraak GJ, Maas SM, Buys CH 1998 Oncological implications of RET gene mutations in Hirschsprung's disease. Gut 43:542-547.

75. Asai N, Jijiwa M, Enomoto A, Kawai K, Maeda K, Ichiahara M, Murakumo Y, Takahashi M 2006 RET receptor signaling: dysfunction in thyroid cancer and Hirschsprung's disease. Pathol Int 56:164-172.

76. Smith DP, Houghton C, Ponder BA 1997 Germline mutation of RET codon 883 in two cases of de novo MEN 2B. Oncogene 15:1213-1217.

77. Gimm O, Marsh DJ, Andrew SD, Frilling A, Dahia PL, Mulligan LM, Zajac JD, Robinson BG, Eng C 1997 Germline dinucleotide mutation in codon 883 of the RET proto-oncogene in multiple endocrine neoplasia type $2 \mathrm{~B}$ without codon 918 mutation. J Clin Endocrinol Metab 82:3902-3904.

78. Jasim S, Ying AK, Waguespack SG, Rich TA, Grubbs EG, Jimenez C, Hu MI, Cote G, Habra MA 2011 Multiple endocrine neoplasia type $2 \mathrm{~B}$ with a RET proto-oncogene 
A883F mutation displays a more indolent form of medullary thyroid carcinoma compared with a RET M918T mutation. Thyroid 21:189-192.

79. Smith VV, Eng C, Milla PJ 1999 Intestinal ganglioneuromatosis and multiple endocrine neoplasia type 2B: implications for treatment. Gut 45:143-146.

80. Cohen MS, Phay JE, Albinson C, DeBenedetti MK, Skinner MA, Lairmore TC, Doherty GM, Balfe DM, Wells SA Jr, Moley JF 2002 Gastrointestinal manifestations of multiple endocrine neoplasia type 2. Ann Surg 235:648-654; discussion 654-655.

81. Waguespack SG, Rich TA, Perrier ND, Jimenez C, Cote GJ 2011 Management of medullary thyroid carcinoma and MEN2 syndromes in childhood. Nat Rev Endocrinol 7: 596-607.

82. Miyauchi A, Futami H, Hai N, Yokozawa T, Kuma K, Aoki N, Kosugi S, Sugano K, Yamaguchi K 1999 Two germline missense mutations at codons 804 and 806 of the RET proto-oncogene in the same allele in a patient with multiple endocrine neoplasia type 2B without codon 918 mutation. Jpn J Cancer Res 90:1-5.

83. Iwashita $\mathrm{T}$, Murakami $\mathrm{H}$, Kurokawa $\mathrm{K}$, Kawai $\mathrm{K}$, Miyauchi A, Futami H, Qiao S, Ichihara M, Takahashi M 2000 A two-hit model for development of multiple endocrine neoplasia type $2 \mathrm{~B}$ by RET mutations. Biochem Biophys Res Commun 268:804-808.

84. Kameyama K, Okinaga H, Takami H 2004 RET oncogene mutations in 75 cases of familial medullary thyroid carcinoma in Japan. Biomed Pharmacother 58:345-347.

85. Nakao KT, Usui T, Ikeda M, Mori Y, Yamamoto T, Kawashima ST, Nanba K, Yuno A, Tamanaha T, Tagami T, Naruse M, Asato R, Shimatsu A 2013 Novel tandem germline RET proto-oncogene mutations in a patient with multiple endocrine neoplasia type 2B: report of a case and a literature review of tandem RET mutations with in silico analysis. Head Neck 35:E363-E368.

86. Bartsch DK, Hasse C, Schug C, Barth P, Rothmund M, Hoppner W 2000 A RET double mutation in the germline of a kindred with FMTC. Exp Clin Endocrinol Diabetes 108: $128-132$.

87. Kasprzak L, Nolet S, Gaboury L, Pavia C, Villabona C, Rivera-Fillat F, Oriola J, Foulkes WD 2001 Familial medullary thyroid carcinoma and prominent corneal nerves associated with the germline V804M and V778I mutations on the same allele of RET. J Med Genet 38: 784-787.

88. 2012 Gene Tests: Medical Genetics Information Resource [database online]. www.genetest.org

89. Conzo G, Circelli L, Pasquali D, Sinisi A, Sabatino L, Accardo G, Renzullo A, Santini L, Salvatore F, Colantuoni V 2012 Lessons to be learned from the clinical management of a MEN 2A patient bearing a novel 634/ 640/700 mutation of the RET proto-oncogene. Clin Endocrinol (Oxf) 77:934-936.

90. Qi XP, Ma JM, Du ZF, Ying RB, Fei J, Jin HY, Han JS, Wang JQ, Chen XL, Chen CY, Liu WT, Lu JJ, Zhang JG, Zhang XN 2011 RET germline mutations identified by exome sequencing in a Chinese multiple endocrine neoplasia type $2 \mathrm{~A} /$ familial medullary thyroid carcinoma family. PLoS One 6:e20353.

91. Dvorakova S, Vaclavikova E, Ryska A, Cap J, Vlcek P, Duskova J, Kodetova D, Holub V, Novak Z, Bendlova B 2006 Double germline mutations in the RET Proto- oncogene in MEN 2A and MEN 2B kindreds. Exp Clin Endocrinol Diabetes 114:192-196.

92. Poturnajova M, Altanerova V, Kostalova L, Breza J, Altaner C 2005 Novel germline mutation in the transmembrane region of RET gene close to Cys634Ser mutation associated with MEN 2A syndrome. J Mol Med (Berl) 83:287-295.

93. Nunes AB, Ezabella MC, Pereira AC, Krieger JE, Toledo SP 2002 A novel Val648Ile substitution in RET protooncogene observed in a Cys634Arg multiple endocrine neoplasia type $2 \mathrm{~A}$ kindred presenting with an adrenocorticotropin-producing pheochromocytoma. J Clin Endocrinol Metab 87:5658-5661.

94. Cerutti JM, Maciel RM 2013 An unusual genotypephenotype correlation in MEN 2 patients: should screening for RET double germline mutations be performed to avoid misleading diagnosis and treatment? Clin Endocrinol (Oxf) 79:591-592.

95. Valente FO, Dias da Silva MR, Camacho CP, Kunii IS, Bastos AU, da Fonseca CC, Simiao HP, Tamanaha R, Maciel RM, Cerutti JM 2013 Comprehensive analysis of RET gene should be performed in patients with multiple endocrine neoplasia type 2 (MEN 2) syndrome and no apparent genotype-phenotype correlation: an appraisal of p.Y791F and p.C634Y RET mutations in five unrelated Brazilian families. J Endocrinol Invest 36:975-981.

96. Elisei R, Romei C, Cosci B, Agate L, Bottici V, Molinaro E, Sculli M, Miccoli P, Basolo F, Grasso L, Pacini F, Pinchera A 2007 RET genetic screening in patients with medullary thyroid cancer and their relatives: experience with 807 individuals at one center. J Clin Endocrinol Metab 92:4725-4729.

97. Eng C, Mulligan LM, Smith DP, Healey CS, Frilling A, Raue F, Neumann HP, Ponder MA, Ponder BA 1995 Low frequency of germline mutations in the RET protooncogene in patients with apparently sporadic medullary thyroid carcinoma. Clin Endocrinol (Oxf) 43:123-127.

98. Carlson KM, Bracamontes J, Jackson CE, Clark R, Lacroix A, Wells SA Jr, Goodfellow PJ 1994 Parent-oforigin effects in multiple endocrine neoplasia type $2 \mathrm{~B}$. Am J Hum Genet 55:1076-1082.

99. Schuffenecker I, Ginet N, Goldgar D, Eng C, Chambe B, Boneu A, Houdent C, Pallo D, Schlumberger M, Thivolet C, Lenoir GM 1997 Prevalence and parental origin of de novo RET mutations in multiple endocrine neoplasia type 2A and familial medullary thyroid carcinoma. Le Groupe d'Etude des Tumeurs a Calcitonine. Am J Hum Genet 60:233-237.

100. Tarasoff v. Regents of the University of California, 551 P.2d, 334 (CA 1976).

101. Pate v Threlkel, 661 So.2d 278 (Fla 1995).

102. Rosenthal MS, Pierce HH 2005 Inherited medullary thyroid cancer and the duty to warn: revisiting Pate v. Threlkel in light of HIPAA. Thyroid 15:140-145.

103. Laberge AM, Burke W 2009 Duty to warn at-risk family members of genetic disease. Virtual Mentor 11:656-660.

104. Sulmasy DP 2000 On warning families about genetic risk: the ghost of Tarasoff. Am J Med 109:738-739.

105. American Medical Association 2003 Opinion 2.131: Disclosure of familial risk in genetic testing. Code of medical ethics. Available at www.ama-assn.org/ama/pub/physicianresources/medical-ethics/code-medical-ethics/opinion2131 .page? 
106. Robson ME, Storm CD, Weitzel J, Wollins DS, Offit K 2010 American Society of Clinical Oncology policy statement update: genetic and genomic testing for cancer susceptibility. J Clin Oncol 28:893-901.

107. 1998 ASHG statement. Professional disclosure of familial genetic information. The American Society of Human Genetics Social Issues Subcommittee on Familial Disclosure. Am J Hum Genet 62:474-483.

108. Green RC, Berg JS, Grody WW, Kalia SS, Korf BR, Martin CL, McGuire AL, Nussbaum RL, O'Daniel JM, Ormond KE, Rehm HL, Watson MS, Williams MS, Biesecker LG; American College of Medical Genetics and Genomics 2013 ACMG recommendations for reporting of incidental findings in clinical exome and genome sequencing. Genet Med 15:565-574.

109. Gostin LO 2001 National health information privacy: regulations under the Health Insurance Portability and Accountability Act. JAMA 285:3015-3021.

110. Fleisher LD, Cole LJ 2001 Health Insurance Portability and Accountability Act is here: what price privacy? Genet Med 3:286-289.

111. Rosenthal MS, Diekema DS 2011 Pediatric ethics guidelines for hereditary medullary thyroid cancer. Int J Pediatr Endocrinol 2011:847603.

112. 1995 Informed consent, parental permission, and assent in pediatric practice. Committee on Bioethics, American Academy of Pediatrics. Pediatrics 95:314-317.

113. Huang SM, Tao BL, Tzeng CC, Liu HT, Wang WP 1997 Prenatal molecular diagnosis of RET proto-oncogene mutation in multiple endocrine neoplasia type $2 \mathrm{~A}$. J Formos Med Assoc 96:542-544.

114. Martinelli P, Maruotti GM, Pasquali D, Paladini D, Agangi A, Rippa E, Colantuoni V, Bellastella A, Sinisi AA 2004 Genetic prenatal RET testing and pregnancy management of multiple endocrine neoplasia Type II A (MEN2A): a case report. J Endocrinol Invest 27:357-360.

115. Offit K, Kohut K, Clagett B, Wadsworth EA, Lafaro KJ, Cummings S, White M, Sagi M, Bernstein D, Davis JG 2006 Cancer genetic testing and assisted reproduction. J Clin Oncol 24:4775-4782.

116. Offit K, Sagi M, Hurley K 2006 Preimplantation genetic diagnosis for cancer syndromes: a new challenge for preventive medicine. JAMA 296:2727-2730.

117. Fan HC, Gu W, Wang J, Blumenfeld YJ, El-Sayed YY, Quake SR 2012 Non-invasive prenatal measurement of the fetal genome. Nature 487:320-324.

118. Macher HC, Martinez-Broca MA, Rubio-Calvo A, LeonGarcia C, Conde-Sanchez M, Costa A, Navarro E, Guerrero JM 2012 Non-invasive prenatal diagnosis of multiple endocrine neoplasia type 2A using COLD-PCR combined with HRM genotyping analysis from maternal serum. PLoS One 7:e51024.

119. Lietman SA 2011 Preimplantation genetic diagnosis for hereditary endocrine disease. Endocr Pract 17(Suppl 3): 28-32.

120. Martin J, Cervero A, Mir P, Martinez-Conejero JA, Pellicer A, Simón C 2013 The impact of next-generation sequencing technology on preimplantation genetic diagnosis and screening. Fertil Steril 99:1054-1061.e3.

121. Altarescu G, Barenholz O, Renbaum P, Beeri R, LevyLahad E, Margalioth EJ, Brooks B, Varshaver I, EldarGeva T 2011 Preimplantation genetic diagnosis (PGD) prevention of the birth of children affected with endocrine diseases. J Pediatr Endocrinol Metab 24:543-548.
122. Chervenak FA, McCullough LB 1996 The fetus as a patient: an essential ethical concept for maternal-fetal medicine. J Matern Fetal Med 5:115-119.

123. Abe K, Adachi I, Miyakawa S, Tanaka M, Yamaguchi K, Tanaka N, Kameya T, Shimosato Y 1977 Production of calcitonin, adrenocorticotropic hormone, and betamelanocyte-stimulating hormone in tumors derived from amine precursor uptake and decarboxylation cells. Cancer Res 37:4190-4194.

124. Baylin SB, Beaven MA, Engelman K, Sjoerdsma A 1970 Elevated histaminase activity in medullary carcinoma of the thyroid gland. N Engl J Med 283:1239-1244.

125. Hirsch PF, Gauthier GF, Munson PL 1963 Thyroid hypocalcemic principle and recurrent laryngeal nerve injury as factors affecting the response to parathyroidectomy in rats. Endocrinology 73:244-252.

126. Ishikawa N, Hamada S 1976 Association of medullary carcinoma of the thyroid with carcinoembryonic antigen. Br J Cancer 34:111-115.

127. Zeytinoglu FN, Gagel RF, Tashjian AH Jr, Hammer RA, Leeman SE 1980 Characterization of neurotensin production by a line of rat medullary thyroid carcinoma cells. Proc Natl Acad Sci U S A 77:3741-3745.

128. Mato E, Matias-Guiu X, Chico A, Webb SM, Cabezas R, Berna L, De Leiva A 1998 Somatostatin and somatostatin receptor subtype gene expression in medullary thyroid carcinoma. J Clin Endocrinol Metab 83:2417-2420.

129. Costante G, Durante C, Francis Z, Schlumberger M, Filetti S 2009 Determination of calcitonin levels in C-cell disease: clinical interest and potential pitfalls. Nat Clin Pract Endocrinol Metab 5:35-44.

130. Becker KL, Nylén ES, White JC, Müller B, Snider RH Jr 2004 Clinical review 167: Procalcitonin and the calcitonin gene family of peptides in inflammation, infection, and sepsis: a journey from calcitonin back to its precursors. $\mathbf{J}$ Clin Endocrinol Metab 89:1512-1525.

131. Whang KT, Steinwald PM, White JC, Nylen ES, Snider RH, Simon GL, Goldberg RL, Becker KL 1998 Serum calcitonin precursors in sepsis and systemic inflammation. J Clin Endocrinol Metab 83:3296-3301.

132. Borchhardt KA, Horl WH, Sunder-Plassmann G 2005 Reversibility of 'secondary hypercalcitoninemia' after kidney transplantation. Am J Transplant 5:1757-1763.

133. Bevilacqua M, Dominguez LJ, Righini V, Valdes V, Vago T, Leopaldi E, Baldi G, Barrella M, Barbagallo M 2006 Dissimilar PTH, gastrin, and calcitonin responses to oral calcium and peptones in hypocalciuric hypercalcemia, primary hyperparathyroidism, and normal subjects: a useful tool for differential diagnosis. J Bone Miner Res 21:406-412.

134. Schuetz M, Duan H, Wahl K, Pirich C, Antoni A, Kommata S, Kletter K, Dudczak R, Karanikas G, Willheim M 2006 T lymphocyte cytokine production patterns in Hashimoto patients with elevated calcitonin levels and their relationship to tumor initiation. Anticancer Res 26:4591-4596.

135. Pratz KW, Ma C, Aubry MC, Vrtiska TJ, Erlichman C 2005 Large cell carcinoma with calcitonin and vasoactive intestinal polypeptide-associated Verner-Morrison syndrome. Mayo Clin Proc 80:116-120.

136. Sim SJ, Glassman AB, Ro JY, Lee JJ, Logothetis CJ, Liu FJ 1996 Serum calcitonin in small cell carcinoma of the prostate. Ann Clin Lab Sci 26:487-495.

137. Machens A, Haedecke J, Holzhausen HJ, Thomusch O, Schneyer U, Dralle H 2000 Differential diagnosis of 
calcitonin-secreting neuroendocrine carcinoma of the foregut by pentagastrin stimulation. Langenbecks Arch Surg 385: 398-401.

138. Yocum MW, Butterfield JH, Gharib H 1994 Increased plasma calcitonin levels in systemic mast cell disease. Mayo Clin Proc 69:987-990.

139. Toledo SP, Lourenco DM Jr, Santos MA, Tavares MR, Toledo RA, Correia-Deur JE 2009 Hypercalcitoninemia is not pathognomonic of medullary thyroid carcinoma. Clinics (Sao Paulo) 64:699-706.

140. Preissner CM, Dodge LA, O'Kane DJ, Singh RJ, Grebe SK 2005 Prevalence of heterophilic antibody interference in eight automated tumor marker immunoassays. Clin Chem 51:208-210.

141. Leboeuf R, Langlois MF, Martin M, Ahnadi CE, Fink GD 2006 "Hook effect" in calcitonin immunoradiometric assay in patients with metastatic medullary thyroid carcinoma: case report and review of the literature. J Clin Endocrinol Metab 91:361-364.

142. d'Herbomez M, Caron P, Bauters C, Do Cao C, Schlienger JL, Sapin R, Baldet L, Carnaille B, Wemeau JL 2007 Reference range of serum calcitonin levels in humans: influence of calcitonin assays, sex, age, and cigarette smoking. Eur J Endocrinol 157:749-755.

143. Basuyau JP, Mallet E, Leroy M, Brunelle P 2004 Reference intervals for serum calcitonin in men, women, and children. Clin Chem 50:1828-1830.

144. Guyetant S, Rousselet MC, Durigon M, Chappard D, Franc B, Guerin O, Saint-Andre JP 1997 Sex-related Ccell hyperplasia in the normal human thyroid: a quantitative autopsy study. J Clin Endocrinol Metab 82:42-47.

145. Algeciras-Schimnich A, Preissner CM, Theobald JP, Finseth MS, Grebe SK 2009 Procalcitonin: a marker for the diagnosis and follow-up of patients with medullary thyroid carcinoma. J Clin Endocrinol Metab 94:861-868.

146. Walter MA, Meier C, Radimerski T, Iten F, Kranzlin M, Muller-Brand J, de Groot JW, Kema IP, Links TP, Muller B 2010 Procalcitonin levels predict clinical course and progression-free survival in patients with medullary thyroid cancer. Cancer 116:31-40.

147. Lorenz K, Elwerr M, Machens A, Abuazab M, Holzhausen HJ, Dralle H 2013 Hypercalcitoninemia in thyroid conditions other than medullary thyroid carcinoma: a comparative analysis of calcium and pentagastrin stimulation of serum calcitonin. Langenbecks Arch Surg 398: 403-409.

148. Trimboli P, Giovanella L, Crescenzi A, Romanelli F, Valabrega S, Spriano G, Cremonini N, Guglielmi R, Papini E 2014 Medullary thyroid cancer diagnosis: an appraisal. Head Neck 36:1216-1233.

149. Colombo C, Verga U, Mian C, Ferrero S, Perrino M, Vicentini L, Dazzi D, Opocher G, Pelizzo MR, BeckPeccoz P, Fugazzola L 2012 Comparison of calcium and pentagastrin tests for the diagnosis and follow-up of medullary thyroid cancer. J Clin Endocrinol Metab 97:905-913.

150. Main C, Perrino MN, Colombo C, Cavedon E, Pennelli G, Ferrero S, De Leo S, Sarais C, Cacciatore C, Manfredi GI, Verga U, Iacobone $\mathrm{M}$, De Pasquale L Pelizzo MR, Vicentini L, Persani L, Fugazzola L 2014 Refining calcium test for the diagnosis of medullary thyroid cancer: cutoffs, procedures, and safety. J Clin Endocrinol Metab 99:1656-1664.

151. Wells SA Jr, Haagensen DE Jr, Linehan WM, Farrell RE, Dilley WG 1978 The detection of elevated plasma levels of carcinoembryonic antigen in patients with suspected or established medullary thyroid carcinoma. Cancer 42: 1498-1503.

152. Mendelsohn G, Wells SA Jr, Baylin SB 1984 Relationship of tissue carcinoembryonic antigen and calcitonin to tumor virulence in medullary thyroid carcinoma. An immunohistochemical study in early, localized, and virulent disseminated stages of disease. Cancer 54:657-662.

153. Bockhorn M, Frilling A, Rewerk S, Liedke M, Dirsch O, Schmid KW, Broelsch CE 2004 Lack of elevated serum carcinoembryonic antigen and calcitonin in medullary thyroid carcinoma. Thyroid 14:468-470.

154. Dora JM, Canalli MH, Capp C, Punales MK, Vieira JG, Maia AL 2008 Normal perioperative serum calcitonin levels in patients with advanced medullary thyroid carcinoma: case report and review of the literature. Thyroid 18:895-899.

155. Frank-Raue K, Machens A, Leidig-Bruckner G, Rondot S, Haag C, Schulze E, Lorenz A, Kreissl MC, Dralle H, Raue F, Schmid KW 2013 Prevalence and clinical spectrum of nonsecretory medullary thyroid carcinoma in a series of 839 patients with sporadic medullary thyroid carcinoma. Thyroid 23:294-300.

156. Khurana R, Agarwal A, Bajpai VK, Verma N, Sharma AK, Gupta RP, Madhusudan KP 2004 Unraveling the amyloid associated with human medullary thyroid carcinoma. Endocrinology 145:5465-5470.

157. Nangue C, Bron L, Portmann L, Volante M, Ris HB, Monnier P, Andrejevic-Blant S 2009 Mixed medullarypapillary carcinoma of the thyroid: report of a case and review of the literature. Head Neck 31:968-974.

158. Griffith C, Zhang S, Mukhopadhyay S 2010 Synchronous metastatic medullary and papillary thyroid carcinomas in a patient with germline RET mutation: case report, molecular analysis, and implications for pathogenesis. Endocr Pathol 21:115-119.

159. Machens A, Dralle H 2012 Simultaneous medullary and papillary thyroid cancer: a novel entity? Ann Surg Oncol 19:37-44.

160. Biscolla RP, Ugolini C, Sculli M, Bottici V, Castagna MG, Romei C, Cosci B, Molinaro E, Faviana P, Basolo F, Miccoli P, Pacini F, Pinchera A, Elisei R 2004 Medullary and papillary tumors are frequently associated in the same thyroid gland without evidence of reciprocal influence in their biologic behavior. Thyroid 14:946-952.

161. Kim WG, Gong G, Kim EY, Kim TY, Hong SJ, Kim WB, Shong YK 2010 Concurrent occurrence of medullary thyroid carcinoma and papillary thyroid carcinoma in the same thyroid should be considered as coincidental. Clin Endocrinol (Oxf) 72:256-263.

162. Pastolero GC, Coire CI, Asa SL 1996 Concurrent medullary and papillary carcinomas of thyroid with lymph node metastases. A collision phenomenon. Am J Surg Pathol 20:245- 250.

163. Kostoglou-Athanassiou I, Athanassiou P, Vecchini G, Gogou L, Kaldrymides P 2004 Mixed medullary-follicular thyroid cancer: report of a case and review of the literature. Horm Res 61:300-304.

164. Papotti M, Komminoth P, Sobrinho-Simoes M, Bussolati G 2000 Thyroid carcinoma with mixed follicular and C-cell differentiation patterns. Semin Diagn Pathol 17: 109-119.

165. Wolfe HJ, Melvin KE, Cervi-Skinner SJ, Saadi AA, Juliar JF, Jackson CE, Tashjian AH Jr 1973 C-cell hyperplasia 
preceding medullary thyroid carcinoma. N Engl $\mathrm{J}$ Med 289:437-441.

166. Mete O, Asa SL 2013 Precursor lesions of endocrine system neoplasms. Pathology 45:316-330.

167. Biddinger PW, Brennan MF, Rosen PP 1991 Symptomatic C-cell hyperplasia associated with chronic lymphocytic thyroiditis. Am J Surg Pathol 15:599-604.

168. LiVolsi VA, Feind CR, LoGerfo P, Tashjian AH Jr 1973 Demonstration by immunoperoxidase staining of hyperplasia of parafollicular cells in the thyroid gland in hyperparathyroidism. J Clin Endocrinol Metab 37:550-559.

169. O’Toole K, Fenoglio-Preiser C, Pushparaj N 1985 Endocrine changes associated with the human aging process: III. Effect of age on the number of calcitonin immunoreactive cells in the thyroid gland. Hum Pathol 16:9911000.

170. Tomita T, Millard DM 1992 C-cell hyperplasia in secondary hyperparathyroidism. Histopathology 21:469-474.

171. Machens A, Dralle H 2012 Biological relevance of medullary thyroid microcarcinoma. J Clin Endocrinol Metab 97:1547-1553.

172. Valenta LJ, Michel-Bechet M, Mattson JC, Singer FR 1977 Microfollicular thyroid carcinoma with amyloid rich stroma resembling the medullary carcinoma of the thyroid (MCT). Cancer 39:1573-1586.

173. Chang TC, Wu SL, Hsiao YL 2005 Medullary thyroid carcinoma: pitfalls in diagnosis by fine needle aspiration cytology and relationship of cytomorphology to RET proto-oncogene mutations. Acta Cytol 49:477-482.

174. Papaparaskeva K, Nagel H, Droese M 2000 Cytologic diagnosis of medullary carcinoma of the thyroid gland. Diagn Cytopathol 22:351-358.

175. Trimboli P, Treglia, G, Guidobaldi L, Romanelli F, Nigri G, Valabrega S, Sadeghi R, Crescenzi A, Faquin WC, Bongiovanni M, Giovanella L 2015 Detection rate of FNA cytology in medullary thyroid carcinoma: a meta-analysis. Clin Endocrinol (Oxf) 82:280-285.

176. Trimboli P, Cremonini N, Ceriani L, Saggiorato E, Guidobaldi L, Romanelli F, Ventura C, Laurenti O, Messuti I, Solaroli E, Madaio R, Bongiovanni M, Orlandi F, Crescenzi A, Valabrega S, Giovanella L 2014 Calcitonin measurement in aspiration needle washout fluids has higher sensitivity than cytology in detecting medullary thyroid cancer: a retrospective multicentre study. Clin Endocrinol 80:135-140.

177. Elisei R, Bottici V, Luchetti F, Di Coscio G, Romei C, Grasso L, Miccoli P, Iacconi P, Basolo F, Pinchera A, Pacini F 2004 Impact of routine measurement of serum calcitonin on the diagnosis and outcome of medullary thyroid cancer: experience in 10,864 patients with nodular thyroid disorders. J Clin Endocrinol Metab 89:163-168.

178. Vierhapper H, Raber W, Bieglmayer C, Kaserer K, Weinhausl A, Niederle B 1997 Routine measurement of plasma calcitonin in nodular thyroid diseases. J Clin Endocrinol Metab 82:1589-1593.

179. Costante G, Meringolo D, Durante C, Bianchi D, Nocera M, Tumino S, Crocetti U, Attard M, Maranghi M, Torlontano M, Filetti S 2007 Predictive value of serum calcitonin levels for preoperative diagnosis of medullary thyroid carcinoma in a cohort of 5817 consecutive patients with thyroid nodules. J Clin Endocrinol Metab 92: 450-455.

180. Cheung K, Roman SA, Wang TS, Walker HD, Sosa JA 2008 Calcitonin measurement in the evaluation of thyroid nodules in the United States: a cost-effectiveness and decision analysis. J Clin Endocrinol Metab 93:2173-2180.

181. Daniels GH 2011 Screening for medullary thyroid carcinoma with serum calcitonin measurements in patients with thyroid nodules in the United States and Canada. Thyroid 21:1199-1207.

182. Boi F, Maurelli I, Pinna G, Atzeni F, Piga M, Lai ML, Mariotti S 2007 Calcitonin measurement in wash-out fluid from fine needle aspiration of neck masses in patients with primary and metastatic medullary thyroid carcinoma. J Clin Endocrinol Metab 92:2115-2118.

183. Kudo T, Miyauchi A, Ito Y, Takamura Y, Amino N, Hirokawa M 2007 Diagnosis of medullary thyroid carcinoma by calcitonin measurement in fine-needle aspiration biopsy specimens. Thyroid 17:635-638.

184. Miyauchi A, Matsuzuka F, Hirai K, Yokozawa T, Kobayashi K, Ito Y, Nakano K, Kuma K, Futami H, Yamaguchi K 2002 Prospective trial of unilateral surgery for nonhereditary medullary thyroid carcinoma in patients without germline RET mutations. World J Surg 26:1023-1028.

185. Machens A, Hauptmann S, Dralle H 2007 Increased risk of lymph node metastasis in multifocal hereditary and sporadic medullary thyroid cancer. World J Surg 31: 1960-1965.

186. Kaserer K, Scheuba C, Neuhold N, Weinhausel A, Haas OA, Vierhapper H, Niederle B 2001 Sporadic versus familial medullary thyroid microcarcinoma: a histopathologic study of 50 consecutive patients. Am J Surg Pathol 25:1245-1251.

187. Machens A, Dralle H 2010 Biomarker-based risk stratification for previously untreated medullary thyroid cancer. $\mathrm{J}$ Clin Endocrinol Metab 95:2655-2663.

188. Giraudet AL, Vanel D, Leboulleux S, Auperin A, Dromain C, Chami L, Ny Tovo N, Lumbroso J, Lassau N, Bonniaud G, Hartl D, Travagli JP, Baudin E, Schlumberger M 2007 Imaging medullary thyroid carcinoma with persistent elevated calcitonin levels. J Clin Endocrinol Metab 92:4185-4190.

189. Weber T, Schilling T, Frank-Raue K, Colombo-Benkmann M, Hinz U, Ziegler R, Klar E 2001 Impact of modified radical neck dissection on biochemical cure in medullary thyroid carcinomas. Surgery 130:1044-1049.

190. Moley JF, DeBenedetti MK 1999 Patterns of nodal metastases in palpable medullary thyroid carcinoma: recommendations for extent of node dissection. Ann Surg 229:880-887; discussion 887-888.

191. Zhang L, Wei WJ, Ji QH, Zhu YX, Wang ZY, Wang Y, Huang CP, Shen Q, Li DS, Wu Y 2012 Risk factors for neck nodal metastasis in papillary thyroid microcarcinoma: a study of 1066 patients. J Clin Endocrinol Metab 97:1250-1257.

192. Park JH, Lee YS, Kim BW, Chang HS, Park CS 2012 Skip lateral neck node metastases in papillary thyroid carcinoma. World J Surg 36:743-747.

193. Machens A, Hauptmann S, Dralle H 2008 Prediction of lateral lymph node metastases in medullary thyroid cancer. Br J Surg 95:586-591.

194. Machens A, Holzhausen HJ, Dralle H 2006 Contralateral cervical and mediastinal lymph node metastasis in medullary thyroid cancer: systemic disease? Surgery 139: 28-32.

195. Franc S, Niccoli-Sire P, Cohen R, Bardet S, Maes B, Murat A, Krivitzky A, Modigliani E; French Medullary 
Study Group 2001 Complete surgical lymph node resection does not prevent authentic recurrences of medullary thyroid carcinoma. Clin Endocrinol (Oxf) 55: 403-409.

196. Machens A, Ukkat J, Hauptmann S, Dralle H 2007 Abnormal carcinoembryonic antigen levels and medullary thyroid cancer progression: a multivariate analysis. Arch Surg 142:289-293; discussion 294.

197. Lorenz K, Abuazab M, Sekulla C, Nguyen-Thanh P, Brauckhoff M, Dralle H 2010 Management of lymph fistulas in thyroid surgery. Langenbecks Arch Surg 395: 911-917.

198. Roh JL, Kim DH, Park CI 2008 Prospective identification of chyle leakage in patients undergoing lateral neck dissection for metastatic thyroid cancer. Ann Surg Oncol 15: 424-429.

199. Sobol S, Jensen C, Sawyer W 2nd, Costiloe P, Thong N 1985 Objective comparison of physical dysfunction after neck dissection. Am J Surg 150:503-509.

200. Pelizzo MR, Boschin IM, Bernante P, Toniato A, Piotto A, Pagetta C, Nibale O, Rampin L, Muzzio PC, Rubello D 2007 Natural history, diagnosis, treatment and outcome of medullary thyroid cancer: 37 years experience on 157 patients. Eur J Surg Oncol 33:493-497.

201. Scollo C, Baudin E, Travagli JP, Caillou B, Bellon N, Leboulleux S, Schlumberger M 2003 Rationale for central and bilateral lymph node dissection in sporadic and hereditary medullary thyroid cancer. J Clin Endocrinol Metab 88:2070-2075.

202. Leggett MD, Chen SL, Schneider PD, Martinez SR 2008 Prognostic value of lymph node yield and metastatic lymph node ratio in medullary thyroid carcinoma. Ann Surg Oncol 15:2493-2499.

203. Machens A, Gimm O, Ukkat J, Hinze R, Schneyer U, Dralle H 2000 Improved prediction of calcitonin normalization in medullary thyroid carcinoma patients by quantitative lymph node analysis. Cancer 88:1909-1915.

204. Machens A, Dralle H 2013 Prognostic impact of N staging in 715 medullary thyroid cancer patients: proposal for a revised staging system. Ann Surg 257:323-329.

205. Brauckhoff M, Machens A, Thanh PN, Lorenz K, Schmeil A, Stratmann M, Sekulla C, Brauckhoff K, Dralle H 2010 Impact of extent of resection for thyroid cancer invading the aerodigestive tract on surgical morbidity, local recurrence, and cancer-specific survival. Surgery 148: 1257-1266.

206. Machens A, Schneyer U, Holzhausen HJ, Dralle H 2005 Prospects of remission in medullary thyroid carcinoma according to basal calcitonin level. J Clin Endocrinol Metab 90:2029-2034.

207. Dralle H, Damm I, Scheumann GF, Kotzerke J, Kupsch E, Geerlings H, Pichlmayr R 1994 Compartment-oriented microdissection of regional lymph nodes in medullary thyroid carcinoma. Surg Today 24:112-121.

208. Machens A, Dralle H 2013 Benefit-risk balance of reoperation for persistent medullary thyroid cancer. Ann Surg 257:751-757.

209. Schneider R, Randolph GW, Sekulla C, Phelan E, Thanh PN, Bucher M, Machens A, Dralle H, Lorenz K 2013 Continuous intraoperative vagus nerve stimulation for identification of imminent recurrent laryngeal nerve injury. Head Neck 35:1591-1598.

210. Olson JA Jr, DeBenedetti MK, Baumann DS, Wells SA Jr 1996 Parathyroid autotransplantation during thyroidec- tomy. Results of long-term follow-up. Ann Surg 223:472478; discussion 478-480.

211. Machens A, Dralle H 2007 Genotype-phenotype based surgical concept of hereditary medullary thyroid carcinoma. World J Surg 31:957-968.

212. Marsh DJ, McDowall D, Hyland VJ, Andrew SD, Schnitzler M, Gaskin EL, Nevell DF, Diamond T, Delbridge L, Clifton-Bligh P, Robinson BG 1996 The identification of false positive responses to the pentagastrin stimulation test in RET mutation negative members of MEN 2A families. Clin Endocrinol (Oxf) 44:213-220.

213. Machens A, Ukkat J, Brauckhoff M, Gimm O, Dralle H 2005 Advances in the management of hereditary medullary thyroid cancer. J Intern Med 257:50-59.

214. Elisei R, Romei C, Renzini G, Bottici V, Cosci B, Molinaro E, Agate L, Cappagli V, Miccoli P, Berti P, Faviana P, Ugolini C, Basolo F, Vitti P, Pinchera A 2012 The timing of total thyroidectomy in RET gene mutation carriers could be personalized and safely planned on the basis of serum calcitonin: 18 years experience at one single center. J Clin Endocrinol Metab 97:426-435.

215. Sosa JA, Tuggle CT, Wang TS, Thomas DC, Boudourakis L, Rivkees S, Roman SA 2008 Clinical and economic outcomes of thyroid and parathyroid surgery in children. $\mathrm{J}$ Clin Endocrinol Metab 93:3058-3065.

216. Tuggle CT, Roman SA, Wang TS, Boudourakis L, Thomas DC, Udelsman R, Ann Sosa J 2008 Pediatric endocrine surgery: who is operating on our children? Surgery 144:869-877; discussion 877.

217. Frank-Raue K, Buhr H, Dralle H, Klar E, Senninger N, Weber T, Rondot S, Hoppner W, Raue F 2006 Long-term outcome in 46 gene carriers of hereditary medullary thyroid carcinoma after prophylactic thyroidectomy: impact of individual RET genotype. Eur J Endocrinol 155:229236.

218. Bihan H, Baudin E, Meas T, Leboulleux S, Al Ghuzlan A, Hannoteaux V, Travagli JP, Valleur P, Guillausseau PJ, Cohen R; French Group of Endocrine Tumors 2012 Role of prophylactic thyroidectomy in RET 790 familial medullary thyroid carcinoma. Head Neck 34:493-498.

219. Machens A, Gimm O, Hinze R, Hoppner W, Boehm BO, Dralle H 2001 Genotype- phenotype correlations in hereditary medullary thyroid carcinoma: oncological features and biochemical properties. J Clin Endocrinol Metab 86:1104-1109.

220. Frohnauer MK, Decker RA 2000 Update on the MEN 2A c804 RET mutation: is prophylactic thyroidectomy indicated? Surgery 128:1052-1057; discussion 1057-1058.

221. Szinnai G, Meier C, Komminoth P, Zumsteg UW 2003 Review of multiple endocrine neoplasia type $2 \mathrm{~A}$ in children: therapeutic results of early thyroidectomy and prognostic value of codon analysis. Pediatrics 111:E132E139.

222. Calva D, O’Dorisio TM, Sue O’Dorisio M, Lal G, Sugg S, Weigel RJ, Howe JR 2009 When is prophylactic thyroidectomy indicated for patients with the RET codon 609 mutation? Ann Surg Oncol 16:2237-2244.

223. Igaz P, Patócs A, Rácz K, Klein I, Váradi A, Esik O 2002 Occurrence of pheochromocytoma in a MEN2A family with codon 609 mutation of the RET proto-oncogene. J Clin Endocrinol Metab 87:2994.

224. Kinlaw WB, Scott SM, Maue RA, Memoli VA, Harris RD, Daniels GH, Porter DM, Belloni DR, Spooner ET, Ernesti MM, Noll WW 2005 Multiple endocrine neoplasia 
2A due to a unique C609S RET mutation presents with pheochromocytoma and reduced penetrance of medullary thyroid carcinoma. Clin Endocrinol (Oxf) 63:676682.

225. Mian C, Barollo S, Zambonin L, Pennelli G, Bernante P, Pelizzo MR, Nacamulli D, Mantero F, Girelli ME, Opocher G 2009 Characterization of the largest kindred with MEN2A due to a Cys609Ser RET mutation. Fam Cancer 8:379-382.

226. Frilling A, Weber F, Tecklenborg C, Broelsch CE 2003 Prophylactic thyroidectomy in multiple endocrine neoplasia: the impact of molecular mechanisms of RET protooncogene. Langenbecks Arch Surg 388:17-26.

227. Skinner MA, Moley JA, Dilley WG, Owzar K, Debenedetti MK, Wells SA Jr 2005 Prophylactic thyroidectomy in multiple endocrine neoplasia type 2A. N Engl J Med 353:1105-1113.

228. Heizmann O, Haecker FM, Zumsteg U, Muller B, Oberholzer M, Oertli D 2006 Presymptomatic thyroidectomy in multiple endocrine neoplasia 2a. Eur J Surg Oncol 32: 98-102.

229. Lallier M, St-Vil D, Giroux M, Huot C, Gaboury L, Oligny L, Desjardins JG 1998 Prophylactic thyroidectomy for medullary thyroid carcinoma in gene carriers of MEN2 syndrome. J Pediatr Surg 33:846-848.

230. Lau GS, Lang BH, Lo CY, Tso A, Garcia-Barcelo MM, Tam PK, Lam KS 2009 Prophylactic thyroidectomy in ethnic Chinese patients with multiple endocrine neoplasia type $2 \mathrm{~A}$ syndrome after the introduction of genetic testing. Hong Kong Med J 15:326-331.

231. Learoyd DL, Gosnell J, Elston MS, Saurine TJ, Richardson AL, Delbridge LW, Aglen JV, Robinson BG 2005 Experience of prophylactic thyroidectomy in multiple endocrine neoplasia type 2A kindreds with RET codon 804 mutations. Clin Endocrinol (Oxf) 63:636-641.

232. Rodriguez GJ, Balsalobre MD, Pomares F, Torregrosa NM, Rios A, Carbonell P, Glower G, Sola J, Tebar J, Parrilla P 2002 Prophylactic thyroidectomy in MEN 2A syndrome: experience in a single center. J Am Coll Surg 195:159-166.

233. Niccoli-Sire P, Murat A, Baudin E, Henry JF, Proye C, Bigorgne JC, Bstandig B, Modigliani E, Morange S, Schlumberger M, Conte-Devolx B 1999 Early or prophylactic thyroidectomy in MEN 2/FMTC gene carriers: results in 71 thyroidectomized patients. The French Calcitonin Tumours Study Group (GETC). Eur J Endocrinol 141:468-474.

234. Rohmer V, Vidal-Trecan G, Bourdelot A, Niccoli P, Murat A, Wemeau JL, Borson-Chazot F, Schvartz C, Tabarin A, Chabre O, Chabrier G, Caron P, Rodien P, Schlumberger M, Baudin E; Groupe Francais des Tumeurs Endocrines 2011 Prognostic factors of disease-free survival after thyroidectomy in 170 young patients with a RET germline mutation: a multicenter study of the Groupe Francais d'Etude des Tumeurs Endocrines. J Clin Endocrinol Metab 96:E509-E518.

235. Niccoli-Sire P, Murat A, Rohmer V, Gibelin H, Chabrier G, Conte-Devolx B, Visset J, Ronceray J, Jaeck D, Henry JF, Proye C, Carnaille B, Kraimps JL 2003 When should thyroidectomy be performed in familial medullary thyroid carcinoma gene carriers with non-cysteine RET mutations? Surgery 134:1029-1036.

236. Machens A, Schneyer U, Holzhausen HJ, Dralle H 2005 Prospects of remission in medullary thyroid carcinoma according to basal calcitonin level. J Clin Endocrinol Metab 90:2029-2034.

237. Machens A, Niccoli-Sire P, Hoegel J, Frank-Raue K, van Vroonhoven TJ, Roeher HD, Wahl RA, Lamesch P, Raue F, Conte-Devolx B, Dralle H; European Multiple Endocrine Neoplasia Study Group 2003 Early malignant progression of hereditary medullary thyroid cancer. N Engl J Med 349:1517-1525.

238. Leboulleux S, Travagli JP, Caillou B, Laplanche A, Bidart JM, Schlumberger M, Baudin E 2002 Medullary thyroid carcinoma as part of a multiple endocrine neoplasia type 2B syndrome: influence of the stage on the clinical course. Cancer 94:44-50.

239. Zenaty D, Aigrain Y, Peuchmaur M, Philippe-Chomette P, Baumann C, Cornelis F, Hugot JP, Chevenne D, Barbu V, Guillausseau PJ, Schlumberger M, Carel JC, Travagli JP, Leger J 2009 Medullary thyroid carcinoma identified within the first year of life in children with hereditary multiple endocrine neoplasia type 2A (codon 634) and 2B. Eur J Endocrinol 160:807-813.

240. Wray CJ, Rich TA, Waguespack SG, Lee JE, Perrier ND, Evans DB 2008 Failure to recognize multiple endocrine neoplasia 2B: more common than we think? Ann Surg Oncol 15:293-301.

241. Brauckhoff M, Machens A, Hess S, Lorenz K, Gimm O, Brauckhoff K, Sekulla C, Dralle H 2008 Premonitory symptoms preceding metastatic medullary thyroid cancer in MEN 2B: an exploratory analysis. Surgery 144:10441050; discussion 1050-1043.

242. Waguespack SG, Rich TA 2010 Multiple endocrine neoplasia [corrected] syndrome type $2 \mathrm{~B}$ in early childhood: long-term benefit of prophylactic thyroidectomy. Cancer 116: 2284 .

243. Brauckhoff M, Machens A, Lorenz K, Bjoro T, Varhaug JE, Dralle H 2014 Surgical curability of medullary thyroid cancer in multiple endocrine neoplasia 2B: a changing perspective. Ann Surg 259:800-806.

244. Brauckhoff M, Gimm O, Weiss CL, Ukkat J, Sekulla C, Brauckhoff K, Thanh PN, Dralle H, 2004 Multiple endocrine neoplasia 2B syndrome due to codon 918 mutation: clinical manifestation and course in early and late onset disease. World J Surg 28:1305-1311.

245. Asari R, Scheuba C, Kaczirek K, Niederle B 2006 Estimated risk of pheochromocytoma recurrence after adrenal-sparing surgery in patients with multiple endocrine neoplasia type 2A. Arch Surg 141:1199-1205; discussion 1205.

246. Brunt LM, Doherty GM, Norton JA, Soper NJ, Quasebarth MA, Moley JF 1996 Laparoscopic adrenalectomy compared to open adrenalectomy for benign adrenal neoplasms. J Am Coll Surg 183:1-10.

247. Miccoli P, Materazzi G, Brauckhoff M, Ambrosini CE, Miccoli M, Dralle H 2011 No outcome differences between a laparoscopic and retroperitoneoscopic approach in synchronous bilateral adrenal surgery. World J Surg 35: 2698-2702.

248. Walz MK, Groeben H, Alesina PF 2010 Single-access retroperitoneoscopic adrenalectomy (SARA) versus conventional retroperitoneoscopic adrenalectomy (CORA): a case-control study. World J Surg 34:1386-1390.

249. Scholten A, Valk GD, Ulfman D, Borel Rinkes IH, Vriens MR 2011 Unilateral subtotal adrenalectomy for pheochromocytoma in multiple endocrine neoplasia type 2 patients: a feasible surgical strategy. Ann Surg 254:1022-1027. 
250. Brauckhoff M, Stock K, Stock S, Lorenz K, Sekulla C, Brauckhoff K, Thanh PN, Gimm O, Spielmann RP, Dralle H 2008 Limitations of intraoperative adrenal remnant volume measurement in patients undergoing subtotal adrenalectomy. World J Surg 32:863-872.

251. Brauckhoff M, Dralle H 2012 Funktionserhaltende Adrenalektomie bei Nebennierentumoren. Chirug 83: 519-527.

252. Machens A, Brauckhoff M, Holzhausen HJ, Thanh PN, Lehnert H, Dralle H 2005 Codon- specific development of pheochromocytoma in multiple endocrine neoplasia type 2. J Clin Endocrinol Metab 90:3999-4003.

253. Rowland KJ, Chernock RD, Moley JF 2013 Pheochromocytoma in an 8-year-old patient with multiple endocrine neoplasia type $2 \mathrm{~A}$ : implications for screening. J Surg Oncol 108:203-206.

254. Nguyen L, Niccoli-Sire P, Caron P, Bastie D, Maes B, Chabrier G, Chabre O, Rohmer V, Lecomte P, Henry JF, Conte-Devolx B; French Calcitonin Tumors Study Group 2001 Pheochromocytoma in multiple endocrine neoplasia type 2: a prospective study. Eur J Endocrinol 144:37-44.

255. Quayle FJ, Fialkowski EA, Benveniste R, Moley JF 2007 Pheochromocytoma penetrance varies by RET mutation in MEN 2A. Surgery 142:800-805; discussion 805.e1.

256. Thosani S, Ayala-Ramirez M, Palmer L, Hu MI, Rich T, Gagel RF, Cote G, Waguespack SG, Habra MA, Jimenez C 2013 The characterization of pheochromocytoma and its impact on overall survival in multiple endocrine neoplasia type 2. J Clin Endocrinol Metab 98:E1813-E1819.

257. Machens A, Lorenz K, Dralle H 2013 Peak incidence of pheochromocytoma and primary hyperparathyroidism in multiple endocrine neoplasia 2: need for age-adjusted biochemical screening. J Clin Endocrinol Metab 98: E336-E345.

258. Modigliani E, Vasen HM, Raue K, Dralle H, Frilling A, Gheri RG, Brandi ML, Limbert E, Niederle B, Forgas L, Rosenberg-Bourgin M, Calmettes C 1995 Pheochromocytoma in multiple endocrine neoplasia type 2: European study. The Euromen Study Group. J Intern Med 238:363367.

259. Schuffenecker I, Virally-Monod M, Brohet R, Goldgar D, Conte-Devolx B, Leclerc L, Chabre O, Boneu A, Caron J, Houdent C, Modigliani E, Rohmer V, Schlumberger M, Eng C, Guillausseau PJ, Lenoir GM 1998 Risk and penetrance of primary hyperparathyroidism in multiple endocrine neoplasia type $2 \mathrm{~A}$ families with mutations at codon 634 of the RET proto-oncogene. Groupe D'etude des Tumeurs a Calcitonine. J Clin Endocrinol Metab 83: 487-491.

260. Jackson MB, Guttenberg M, Hedrick H, Moshang T Jr 2005 Multiple endocrine neoplasia type 2A in a kindred with C634Y mutation. Pediatrics 116:e468-e471.

261. Kraimps JL, Denizot A, Carnaille B, Henry JF, Proye C, Bacourt F, Sarfati E, Dupond JL, Maes B, Travagli JP, Boneu A, Roger P, Houdent C, Barbier J, Modigliani E 1996 Primary hyperparathyroidism in multiple endocrine neoplasia type IIa: retrospective French multicentric study. Groupe d'Etude des Tumeurs a Calcitonine (GETC, French Calcitonin Tumors Study Group), French Association of Endocrine Surgeons. World J Surg 20:808-812; discussion 812-813.

262. Raue F, Kraimps JL, Dralle H, Cougard P, Proye C, Frilling A, Limbert E, Llenas LF, Niederle B 1995 Pri- mary hyperparathyroidism in multiple endocrine neoplasia type 2A. J Intern Med 238:369-373.

263. Carling T, Udelsman R 2005 Parathyroid surgery in familial hyperparathyroid disorders. J Intern Med 257: 27-37.

264. Scholten A, Schreinemakers JM, Pieterman CR, Valk GD, Vriens MR, Borel Rinkes IH 2011 Evolution of surgical treatment of primary hyperparathyroidism in patients with multiple endocrine neoplasia type 2A. Endocr Pract 17: $7-15$.

265. Irvin GL 3rd, Carneiro DM, Solorzano CC 2004 Progress in the operative management of sporadic primary hyperparathyroidism over 34 years. Ann Surg 239:704-708; discussion 708-711.

266. Agarwal A, Waghray A, Gupta S, Sharma R, Milas M 2013 Cryopreservation of parathyroid tissue: an illustrated technique using the cleveland clinic protocol. J Am Coll Surg 216:e1-e9.

267. Luque-Fernandez I, Garcia-Martin A, Luque-Pazos A 2013 Experience with cinacalcet in primary hyperparathyroidism: results after 1 year of treatment. Ther Adv Endocrinol Metab 4:77-81.

268. Peacock M, Bilezikian JP, Klassen PS, Guo MD, Turner SA, Shoback D 2005 Cinacalcet hydrochloride maintains long-term normocalcemia in patients with primary hyperparathyroidism. J Clin Endocrinol Metab 90:135141.

269. Engelbach M, Gorges R, Forst T, Pfutzner A, Dawood R, Heerdt S, Kunt T, Bockisch A, Beyer J 2000 Improved diagnostic methods in the follow-up of medullary thyroid carcinoma by highly specific calcitonin measurements. J Clin Endocrinol Metab 85:1890-1894.

270. Ismailov SI, Piulatova NR 2004 Postoperative calcitonin study in medullary thyroid carcinoma. Endocr Relat Cancer 11:357-363.

271. Elisei R, Pinchera A 2012 Advances in the follow-up of differentiated or medullary thyroid cancer. Nat Rev Endocrinol 8:466-475.

272. Pellegriti G, Leboulleux S, Baudin E, Bellon N, Scollo C, Travagli JP, Schlumberger M 2003 Long-term outcome of medullary thyroid carcinoma in patients with normal postoperative medical imaging. Br J Cancer 88:15371542.

273. Barbot N, Calmettes C, Schuffenecker I, Saint-Andre JP, Franc B, Rohmer V, Jallet P, Bigorgne JC 1994 Pentagastrin stimulation test and early diagnosis of medullary thyroid carcinoma using an immunoradiometric assay of calcitonin: comparison with genetic screening in hereditary medullary thyroid carcinoma. J Clin Endocrinol Metab 78:114-120.

274. Kouvaraki MA, Shapiro SE, Fornage BD, Edeiken-Monro BS, Sherman SI, Vassilopoulou-Sellin R, Lee JE, Evans DB 2003 Role of preoperative ultrasonography in the surgical management of patients with thyroid cancer. Surgery 134:946-954; discussion 954-945.

275. Koopmans KP, de Groot JW, Plukker JT, de Vries EG, Kema IP, Sluiter WJ, Jager PL, Links TP 2008 18F-dihydroxyphenylalanine PET in patients with biochemical evidence of medullary thyroid cancer: relation to tumor differentiation. J Nucl Med 49:524-531.

276. Szakall S Jr, Esik O, Bajzik G, Repa I, Dabasi G, Sinkovics I, Agoston P, Tron L 2002 18F-FDG PET detection of lymph node metastases in medullary thyroid carcinoma. J Nucl Med 43:66-71. 
277. Gourgiotis L, Sarlis NJ, Reynolds JC, VanWaes C, Merino MJ, Pacak K 2003 Localization of medullary thyroid carcinoma metastasis in a multiple endocrine neoplasia type $2 \mathrm{~A}$ patient by $6-[18 \mathrm{~F}]$-fluorodopamine positron emission tomography. J Clin Endocrinol Metab 88:637641.

278. Hoegerle S, Altehoefer C, Ghanem N, Brink I, Moser E, Nitzsche E 2001 18F-DOPA positron emission tomography for tumour detection in patients with medullary thyroid carcinoma and elevated calcitonin levels. Eur J Nucl Med 28:64-71.

279. Rubello D, Rampin L, Nanni C, Banti E, Ferdeghini M, Fanti S, Al-Nahhas A, Gross MD 2008 The role of 18FFDG PET/CT in detecting metastatic deposits of recurrent medullary thyroid carcinoma: a prospective study. Eur $\mathbf{J}$ Surg Oncol 34:581-586.

280. Ong SC, Schoder H, Patel SG, Tabangay-Lim IM, Doddamane I, Gonen M, Shaha AR, Tuttle RM, Shah JP, Larson SM 2007 Diagnostic accuracy of 18F-FDG PET in restaging patients with medullary thyroid carcinoma and elevated calcitonin levels. J Nucl Med 48:501-507.

281. Baudin E, Lumbroso J, Schlumberger M, Leclere J, Giammarile F, Gardet P, Roche A, Travagli JP, Parmentier C 1996 Comparison of octreotide scintigraphy and conventional imaging in medullary thyroid carcinoma. $\mathrm{J}$ Nucl Med 37:912-916.

282. Behr TM, Gratz S, Markus PM, Dunn RM, Hufner M, Schauer A, Fischer M, Munz DL, Becker H, Becker W 1997 Anti-carcinoembryonic antigen antibodies versus somatostatin analogs in the detection of metastatic medullary thyroid carcinoma: are carcinoembryonic antigen and somatostatin receptor expression prognostic factors? Cancer 80:2436-2457.

283. Behr TM, Béhé MP 2002 Cholecystokinin-B/gastrin receptor-targeting peptides for staging and therapy of medullary thyroid cancer and other cholecystokinin-B receptor-expressing malignancies. Semin Nucl Med 32: 97-109.

284. Clarke SE, Lazarus CR, Wraight P, Sampson C, Maisey MN 1988 Pentavalent Tc99m DMSA, I-131 MIBG, and Tc-99m MDP - an evaluation of three imaging techniques in patients with medullary carcinoma of the thyroid. J Nucl Med 29:33-38.

285. Frank-Raue K, Bihl H, Dorr U, Buhr H, Ziegler R, Raue F 1995 Somatostatin receptor imaging in persistent medullary thyroid carcinoma. Clin Endocrinol (Oxf) 42: 31-37.

286. Kwekkeboom DJ, Reubi JC, Lamberts SW, Bruining HA, Mulder AH, Oei HY, Krenning EP 1993 In vivo somatostatin receptor imaging in medullary thyroid carcinoma. J Clin Endocrinol Metab 76:1413-1417.

287. Beheshti M, Pocher S, Vali R, Waldenberger P, Broinger G, Nader M, Kohlfurst S, Pirich C, Dralle H, Langsteger W 2009 The value of 18F-DOPA PET-CT in patients with medullary thyroid carcinoma: comparison with $18 \mathrm{~F}-\mathrm{FDG}$ PET-CT. Eur Radiol 19:1425-1434.

288. Kauhanen S, Schalin-Jantti C, Seppanen M, Kajander S, Virtanen S, Schildt J, Lisinen I, Ahonen A, Heiskanen I, Vaisanen M, Arola J, Korsoff P, Ebeling T, Sane T, Minn H, Valimaki MJ, Nuutila P 2011 Complementary roles of 18F-DOPA PET/CT and 18F-FDG PET/CT in medullary thyroid cancer. J Nucl Med 52:1855-1863.

289. Luster M, Karges W, Zeich K, Pauls S, Verburg FA, Dralle H, Glatting G, Buck AK, Solbach C, Neumaier B,
Reske SN, Mottaghy FM 2010 Clinical value of 18fluorine-fluorodihydroxyphenylalanine positron emission tomography/computed tomography in the follow-up of medullary thyroid carcinoma. Thyroid 20:527-533.

290. Treglia G, Castaldi P, Villani MF, Perotti G, de Waure C, Filice A, Ambrosini V, Cremonini N, Santimaria M, Versari A, Fanti S, Giordano A, Rufini V 2012 Comparison of 18F-DOPA, 18F-FDG and 68Ga-somatostatin analogue PET/CT in patients with recurrent medullary thyroid carcinoma. Eur J Nucl Med Mol Imaging 39: 569-580.

291. Verbeek HH, Plukker JT, Koopmans KP, de Groot JW, Hofstra RM, Muller Kobold AC, van der Horst-Schrivers AN, Brouwers AH, Links TP 2012 Clinical relevance of 18 F- FDG PET and 18F-DOPA PET in recurrent medullary thyroid carcinoma. J Nucl Med 53:1863-1871.

292. Therasse P, Arbuck SG, Eisenhauer EA, Wanders J, Kaplan RS, Rubinstein L, Verweij J, Van Glabbeke M, van Oosterom AT, Christian MC, Gwyther SG 2000 New guidelines to evaluate the response to treatment in solid tumors. European Organization for Research and Treatment of Cancer, National Cancer Institute of the United States, National Cancer Institute of Canada. J Natl Cancer Inst 92:205-216.

293. Barbet J, Campion L, Kraeber-Bodéré F, Chatal JF; GTE Study Group 2005 Prognostic impact of serum calcitonin and carcinoembryonic antigen doubling-times in patients with medullary thyroid carcinoma. J Clin Endocrinol Metab 90:6077-6084.

294. Laure Giraudet A, Al Ghulzan A, Auperin A, Leboulleux $\mathrm{S}$, Chehboun A, Troalen F, Dromain C, Lumbroso J, Baudin E, Schlumberger M 2008 Progression of medullary thyroid carcinoma: assessment with calcitonin and carcinoembryonic antigen doubling times. Eur J Endocrinol 158:239-246.

295. Miyauchi A, Onishi T, Morimoto S, Takai S, Matsuzuka F, Kuma K, Maeda M, Kumahara Y 1984 Relation of doubling time of plasma calcitonin levels to prognosis and recurrence of medullary thyroid carcinoma. Ann Surg 199:461-466.

296. Saad MF, Fritsche HA Jr, Samaan NA 1984 Diagnostic and prognostic values of carcinoembryonic antigen in medullary carcinoma of the thyroid. J Clin Endocrinol Metab 58:889-894.

297. Fialkowski E, DeBenedetti M, Moley J 2008 Long-term outcome of reoperations for medullary thyroid carcinoma. World J Surg 32:754-765.

298. Kebebew E, Kikuchi S, Duh QY, Clark OH 2000 Longterm results of reoperation and localizing studies in patients with persistent or recurrent medullary thyroid cancer. Arch Surg 135:895-901.

299. Tisell LE, Hansson G, Jansson S, Salander H 1986 Reoperation in the treatment of asymptomatic metastasizing medullary thyroid carcinoma. Surgery 99:60-66.

300. Tisell LE, Jansson S 1988 Recent results of reoperative surgery in medullary carcinoma of the thyroid. Wien Klin Wochenschr 100:347-348.

301. Deftos LJ, Stein MF 1980 Radioiodine as an adjunct to the surgical treatment of medullary thyroid carcinoma. J Clin Endocrinol Metab 50:967-968.

302. Hellman DE, Kartchner M, Van Antwerp JD, Salmon SE, Patton DD, O'Mara R 1979 Radioiodine in the treatment of medullary carcinoma of the thyroid. J Clin Endocrinol Metab 48:451-455. 
303. Meijer JA, Bakker LE, Valk GD, de Herder WW, de Wilt JH, Netea-Maier RT, Schaper N, Fliers E, Lips P, Plukker JT, Links TP, Smit JA 2013 Radioactive iodine in the treatment of medullary thyroid carcinoma: a controlled multicenter study. Eur J Endocrinol 168:779-786.

304. Brierley JD, Tsang RW 2008 External beam radiation therapy for thyroid cancer. Endocrinol Metab Clin North Am 37:497-509, xi.

305. Fife KM, Bower M, Harmer CL 1996 Medullary thyroid cancer: the role of radiotherapy in local control. Eur $\mathrm{J}$ Surg Oncol 22:588-591.

306. Martinez SR, Beal SH, Chen A, Chen SL, Schneider PD 2010 Adjuvant external beam radiation for medullary thyroid carcinoma. J Surg Oncol 102:175-178.

307. Schwartz DL, Rana V, Shaw S, Yazbeck C, Ang KK, Morrison WH, Rosenthal DI, Hoff A, Evans DB, Clayman GL, Garden AS, Sherman SI 2008 Postoperative radiotherapy for advanced medullary thyroid cancer-local disease control in the modern era. Head Neck 30:883-888.

308. Terezakis SA, Lee NY 2010 The role of radiation therapy in the treatment of medullary thyroid cancer. J Natl Compr Canc Netw 8:532-540; quiz 541.

309. Brierley J, Tsang R, Simpson WJ, Gospodarowicz M, Sutcliffe S, Panzarella T 1996 Medullary thyroid cancer: analyses of survival and prognostic factors and the role of radiation therapy in local control. Thyroid 6:305-310.

310. Call JA, Caudill JS, McIver B, Foote RL 2013 A role for radiotherapy in the management of advanced medullary thyroid carcinoma: the mayo clinic experience. Rare Tumors 5:e37.

311. Bhandare N, Mendenhall WM 2012 A literature review of late complications of radiation therapy for head and neck cancers: incidence and dose response. J Nucl Med Radiat Ther S2:009. DOI:10.4172/2155-9619.S2-009.

312. Tung WS, Vesely TM, Moley JF 1995 Laparoscopic detection of hepatic metastases in patients with residual or recurrent medullary thyroid cancer. Surgery 118:1024 1029; discussion 1029-1030.

313. Cupisti K, Wolf A, Raffel A, Schott M, Miersch D, Yang Q, Eisenberger CF, Roher HD, Knoefel WT 2007 Longterm clinical and biochemical follow-up in medullary thyroid carcinoma: a single institution's experience over 20 years. Ann Surg 246:815-821.

314. de Groot JW, Plukker JT, Wolffenbuttel BH, Wiggers T, Sluiter WJ, Links TP 2006 Determinants of life expectancy in medullary thyroid cancer: age does not matter. Clin Endocrinol (Oxf) 65:729-736.

315. Rendl G, Manzl M, Hitzl W, Sungler P, Pirich C 2008 Long-term prognosis of medullary thyroid carcinoma. Clin Endocrinol (Oxf) 69:497-505.

316. Börcek P, Asa SL, Gentili F, Ezzat S, Kiehl TR 2010 Brain metastasis from medullary thyroid carcinoma. BMJ Case Rep pii:bcr0920103301.

317. Kim IY, Kondziolka D, Niranjan A, Flickinger JC, Lunsford LD 2009 Gamma knife radiosurgery for metastatic brain tumors from thyroid cancer. J Neurooncol 93: 355-359.

318. McWilliams RR, Giannini C, Hay ID, Atkinson JL, Stafford SL, Buckner JC 2003 Management of brain metastases from thyroid carcinoma: a study of 16 pathologically confirmed cases over 25 years. Cancer 98: $356-362$.
319. Wexler JA 2011 Approach to the thyroid cancer patient with bone metastases. J Clin Endocrinol Metab 96: 2296-2307.

320. Quan GM, Pointillart V, Palussiere J, Bonichon F 2012 Multidisciplinary treatment and survival of patients with vertebral metastases from thyroid carcinoma. Thyroid 22: 125-130.

321. Frassica DA 2003 General principles of external beam radiation therapy for skeletal metastases. Clin Orthop Relat Res 145:S158-164.

322. Vitale G, Fonderico F, Martignetti A, Caraglia M, Ciccarelli A, Nuzzo V, Abbruzzese A, Lupoli G 2001 Pamidronate improves the quality of life and induces clinical remission of bone metastases in patients with thyroid cancer. Br J Cancer 84:1586-1590.

323. Abrahamsen B, Eiken P, Eastell R 2009 Subtrochanteric and diaphyseal femur fractures in patients treated with alendronate: a register-based national cohort study. J Bone Miner Res 24:1095-1102.

324. Khosla S, Burr D, Cauley J, Dempster DW, Ebeling PR, Felsenberg D, Gagel RF, Gilsanz V, Guise T, Koka S, McCauley LK, McGowan J, McKee MD, Mohla S, Pendrys DG, Raisz LG, Ruggiero SL, Shafer DM, Shum L, Silverman SL, Van Poznak CH, Watts N, Woo SB, Shane E 2007 Bisphosphonate-associated osteonecrosis of the jaw: report of a task force of the American Society for Bone and Mineral Research. J Bone Miner Res 22: 1479-1491.

325. Tsutsui H, Kubota M, Yamada M, Suzuki A, Usuda J, Shibuya H, Miyajima K, Sugino K, Ito K, Furukawa K, Kato H 2008 Airway stenting for the treatment of laryngotracheal stenosis secondary to thyroid cancer. Respirology 13:632-638.

326. Fromigue J, De Baere $\mathrm{T}$, Baudin E, Dromain C, Leboulleux S, Schlumberger M 2006 Chemoembolization for liver metastases from medullary thyroid carcinoma. J Clin Endocrinol Metab 91:2496-2499.

327. Isozaki T, Kiba T, Numata K, Saito S, Shimamura T, Kitamura T, Morita K, Tanaka K, Sekihara H 1999 Medullary thyroid carcinoma with multiple hepatic metastases: treatment with transcatheter arterial embolization and percutaneous ethanol injection. Intern Med 38: $17-21$.

328. Lorenz K, Brauckhoff M, Behrmann C, Sekulla C, Ukkat J, Brauckhoff K, Gimm O, Dralle H 2005 Selective arterial chemoembolization for hepatic metastases from medullary thyroid carcinoma. Surgery 138:986-993; discussion 993.

329. Wertenbroek MW, Links TP, Prins TR, Plukker JT, van der Jagt EJ, de Jong KP 2008 Radiofrequency ablation of hepatic metastases from thyroid carcinoma. Thyroid 18: $1105-1110$

330. Santarpia L, El-Naggar AK, Sherman SI, Hymes SR, Gagel RF, Shaw S, Sarlis NJ 2008 Four patients with cutaneous metastases from medullary thyroid cancer. Thyroid 18:901- 905.

331. Bajetta E, Rimassa L, Carnaghi C, Seregni E, Ferrari L, Di Bartolomeo M, Regalia E, Cassata A, Procopio G, Mariani L 1998 5-Fluorouracil, dacarbazine, and epirubicin in the treatment of patients with neuroendocrine tumors. Cancer 83:372-378.

332. Orlandi F, Caraci P, Berruti A, Puligheddu B, Pivano G, Dogliotti L, Angeli A 1994 Chemotherapy with dacarba- 
zine and 5-fluorouracil in advanced medullary thyroid cancer. Ann Oncol 5:763-765.

333. Petursson SR 1988 Metastatic medullary thyroid carcinoma. Complete response to combination chemotherapy with dacarbazine and 5-fluorouracil. Cancer 62:18991903.

334. Iten F, Muller B, Schindler C, Rochlitz C, Oertli D, Macke HR, Muller-Brand J, Walter MA 2007 Response to [90Yttrium-DOTA]-TOC treatment is associated with long-term survival benefit in metastasized medullary thyroid cancer: a phase II clinical trial. Clin Cancer Res 13:6696-6702.

335. Chatal JF, Campion L, Kraeber-Bodere F, Bardet S, Vuillez JP, Charbonnel B, Rohmer V, Chang CH, Sharkey RM, Goldenberg DM, Barbet J; French Endocrine Tumor Group 2006 Survival improvement in patients with medullary thyroid carcinoma who undergo pretargeted anti-carcinoembryonic-antigen radioimmunotherapy: a collaborative study with the French Endocrine Tumor Group. J Clin Oncol 24:1705-1711.

336. Kraeber-Bodere F, Rousseau C, Bodet-Milin C, Ferrer L, Faivre-Chauvet A, Campion L, Vuillez JP, Devillers A, Chang CH, Goldenberg DM, Chatal JF, Barbet J 2006 Targeting, toxicity, and efficacy of 2-step, pretargeted radioimmunotherapy using a chimeric bispecific antibody and 131I-labeled bivalent hapten in a phase I optimization clinical trial. J Nucl Med 47:247-255.

337. Maiza JC, Grunenwald S, Otal P, Vezzosi D, Bennet A, Caron P 2012 Use of 131I-MIBG therapy in MIBGpositive metastatic medullary thyroid carcinoma. Thyroid 22:654-655.

338. Pasieka JL, McEwan AJ, Rorstad O 2004 The palliative role of 131I-MIBG and $111 \mathrm{In}$ - octreotide therapy in patients with metastatic progressive neuroendocrine neoplasms. Surgery 136:1218-1226.

339. Capp C, Wajner SM, Siqueira DR, Brasil BA, Meurer L, Maia AL 2010 Increased expression of vascular endothelial growth factor and its receptors, VEGFR-1 and VEGFR-2, in medullary thyroid carcinoma. Thyroid 20: 863-871.

340. Schlumberger MJ, Elisei R, Bastholt L, Wirth LJ, Martins RG, Locati LD, Jarzab B, Pacini F, Daumerie C, Droz JP, Eschenberg MJ, Sun YN, Juan T, Stepan DE, Sherman SI 2009 Phase II study of safety and efficacy of motesanib in patients with progressive or symptomatic, advanced or metastatic medullary thyroid cancer. J Clin Oncol 27: 3794-3801.

341. Cohen EE, Rosen LS, Vokes EE, Kies MS, Forastiere AA, Worden FP, Kane MA, Sherman E, Kim S, Bycott P, Tortorici M, Shalinsky DR, Liau KF, Cohen RB 2008 Axitinib is an active treatment for all histologic subtypes of advanced thyroid cancer: results from a phase II study. J Clin Oncol 26:4708-4713.

342. de Groot JW, Zonnenberg BA, van Ufford-Mannesse PQ, de Vries MM, Links TP, Lips CJ, Voest EE 2007 A phase II trial of imatinib therapy for metastatic medullary thyroid carcinoma. J Clin Endocrinol Metab 92:3466-3469.

343. Frank-Raue K, Fabel M, Delorme S, Haberkorn U, Raue F 2007 Efficacy of imatinib mesylate in advanced medullary thyroid carcinoma. Eur J Endocrinol 157:215-220.

344. Kurzrock R, Sherman SI, Ball DW, Forastiere AA, Cohen RB, Mehra R, Pfister DG, Cohen EE, Janisch L, Nauling F, Hong DS, Ng CS, Ye L, Gagel RF, Frye J, Muller T, Ratain MJ, Salgia R 2011 Activity of XL184 (Cabo- zantinib), an oral tyrosine kinase inhibitor, in patients with medullary thyroid cancer. J Clin Oncol 29:2660-2666.

345. Lam ET, Ringel MD, Kloos RT, Prior TW, Knopp MV, Liang J, Sammet S, Hall NC, Wakely PE Jr, Vasko VV, Saji M, Snyder PJ, Wei L, Arbogast D, Collamore M, Wright JJ, Moley JF, Villalona-Calero MA, Shah MH 2010 Phase II clinical trial of sorafenib in metastatic medullary thyroid cancer. J Clin Oncol 28:2323-2330.

346. Pennell NA, Daniels GH, Haddad RI, Ross DS, Evans T, Wirth LJ, Fidias PH, Temel JS, Gurubhagavatula S, Heist RS, Clark JR, Lynch TJ 2008 A phase II study of gefitinib in patients with advanced thyroid cancer. Thyroid 18: 317-323.

347. Wells SA Jr, Gosnell JE, Gagel RF, Moley J, Pfister D, Sosa JA, Skinner M, Krebs A, Vasselli J, Schlumberger M 2010 Vandetanib for the treatment of patients with locally advanced or metastatic hereditary medullary thyroid cancer. J Clin Oncol 28:767-772.

348. Robinson BG, Paz-Ares L, Krebs A, Vasselli J, Haddad R 2010 Vandetanib $(100 \mathrm{mg})$ in patients with locally advanced or metastatic hereditary medullary thyroid cancer. J Clin Endocrinol Metab 95:2664-2671.

349. Wells SA Jr, Robinson BG, Gagel RF, Dralle H, Fagin JA, Santoro M, Baudin E, Elisei R, Jarzab B, Vasselli JR, Read J, Langmuir P, Ryan AJ, Schlumberger MJ 2012 Vandetanib in patients with locally advanced or metastatic medullary thyroid cancer: a randomized, double-blind phase III trial. J Clin Oncol 30:134-141.

350. Elisei R, Schlumberger MJ, Müller SP, Schöffski P, Brose MS, Shah MH, Licitra L, Jarzab B, Medvedev V, Kreissl MC, Niederle B, Cohen EE, Wirth LJ, Ali H, Hessel C, Yaron Y, Ball D, Nelkin B, Sherman SI 2013 Cabozantinib in progressive medullary thyroid cancer. J Clin Oncol 31:3639-3646.

351. Sherman SI, Cohen EEW, Schoffski P, Elisei R, Schlumberger M, Wirth LJ, Mangeshkar M, Aftab DT, Clary DO, Brose MS 2013 Efficacy of cabozantinib (Cabo) in medullary thyroid cancer (MTC) patients with RAS or RET mutations: results from a phase III study. J Clin Oncol 31: abstract 6000.

352. Brassard M, Neraud B, Trabado S, Salenave S, BraillyTabard S, Borget I, Baudin E, Leboulleux S, Chanson P, Schlumberger M, Young J 2011 Endocrine effects of the tyrosine kinase inhibitor vandetanib in patients treated for thyroid cancer. J Clin Endocrinol Metab 96:27412749.

353. Abdulrahman RM, Verloop H, Hoftijzer H, Verburg E, Hovens GC, Corssmit EP, Reiners C, Gelderblom H, Pereira AM, Kapiteijn E, Romijn JA, Visser TJ, Smit JW 2010 Sorafenib-induced hypothyroidism is associated with increased type 3 deiodination. J Clin Endocrinol Metab 95:3758-3762.

354. Pinto A 2014 Cabozantinib: a novel agent with a dual mechanism of action for castration-resistant prostate carcinoma. Cancer Chemother Pharmacol 73:219-222.

355. Cox TM, Fagan EA, Hillyard CJ, Allison DJ, Chadwick VS 1979 Role of calcitonin in diarrhoea associated with medullary carcinoma of the thyroid. Gut 20:629-633.

356. Rambaud JC, Jian R, Flourie B, Hautefeuille M, Salmeron M, Thuillier F, Ruskone A, Florent C, Chaoui F, Bernier JJ 1988 Pathophysiological study of diarrhoea in a patient with medullary thyroid carcinoma. Evidence against a secretory mechanism and for the role of shortened colonic transit time. Gut 29:537-543. 
357. Mahler C, Verhelst J, de Longueville M, Harris A 1990 Long-term treatment of metastatic medullary thyroid carcinoma with the somatostatin analogue octreotide. Clin Endocrinol (Oxf) 33:261-269.

358. Vainas I, Koussis C, Pazaitou-Panayiotou K, Drimonitis A, Chrisoulidou A, Iakovou I, Boudina M, Kaprara A, Maladaki A 2004 Somatostatin receptor expression in vivo and response to somatostatin analog therapy with or without other antineoplastic treatments in advanced medullary thyroid carcinoma. J Exp Clin Cancer Res 23:549-559.

359. Lupoli G, Cascone E, Arlotta F, Vitale G, Celentano L, Salvatore M, Lombardi G 1996 Treatment of advanced medullary thyroid carcinoma with a combination of recombinant interferon alpha-2b and octreotide. Cancer 78:1114-1118.

360. Vitale G, Tagliaferri P, Caraglia M, Rampone E, Ciccarelli A, Bianco AR, Abbruzzese A, Lupoli G 2000 Slow release lanreotide in combination with interferon-alpha2b in the treatment of symptomatic advanced medullary thyroid carcinoma. J Clin Endocrinol Metab 85:983-988.

361. Ilias I, Torpy DJ, Pacak K, Mullen N, Wesley RA, Nieman LK 2005 Cushing's syndrome due to ectopic corticotropin secretion: twenty years' experience at the National Institutes of Health. J Clin Endocrinol Metab 90: 4955-4962.

362. Wajchenberg BL, Mendonca BB, Liberman B, Pereira MA, Carneiro PC, Wakamatsu A, Kirschner MA 1994
Ectopic adrenocorticotropic hormone syndrome. Endocr Rev 15:752-787.

363. Pozza C, Graziadio C, Giannetta E, Lenzi A, Isidori AM 2012 Management strategies for aggressive Cushing's syndrome: from macroadenomas to ectopics. J Oncol 2012:685213.

364. Baudry C, Paepegaey AC, Groussin L 2013 Reversal of Cushing's syndrome by vandetanib in medullary thyroid carcinoma. N Engl J Med 369:584-586.

365. Fox E, Widemann BC, Chuk MK, Marcus L, Aikin A, Whitcomb PO, Merino MJ, Lodish M, Dombi E, Steinberg SM, Wells SA, Balis FM 2013 Vandetanib in children and adolescents with multiple endocrine neoplasia type 2B associated medullary thyroid carcinoma. Clin Cancer Res 19:4239-4248.

Address correspondence to: Samuel A. Wells, Jr., MD National Cancer Institute National Institutes of Health Cancer Genetics Branch, Bldg. 37, Room 6138 37 Convent Drive Bethesda, MD 20814

E-mail: wellss@mail.nih.gov 